\title{
Characterization of the cell wall protein Ecm33 family in Candida glabrata
}

\author{
Dissertation \\ for the award of the degree \\ "Doctor rerum naturalium" (Dr.rer.nat.) \\ of the Georg-August-Universität Göttingen \\ within the doctoral program Biology \\ of the Georg-August University School of Science (GAUSS)
}

Submitted by

Marut Tangwattanachuleeporn

Born in

Bangkok, Thailand

Göttingen, 2013 


\section{Thesis Committee}

1. Prof. Dr. med. Uwe Groß

(Institute for Medical Microbiology, University Medical Center Göttingen)

2. Prof. Dr. Gerhard H. Braus

(Institute of Microbiology and Genetics, Georg-August-University Göttingen)

\section{$\underline{\text { Supervisor }}$}

PD. Dr. med. Michael Weig

(Institute for Medical Microbiology, University Medical Center Göttingen)

\section{Members of the Examination Board}

Reviewer: Prof. Dr. med. Uwe Groß

(Institute for Medical Microbiology, University Medical Center Göttingen)

Second reviewer: Prof. Dr. Gerhard H. Braus

(Institute of Microbiology and Genetics, Georg-August-University Göttingen)

\section{Further members of the Examination Board:}

1. PD. Dr. Stefan Irniger

(Institute of Microbiology and Genetics, Georg-August-University Göttingen)

2. Prof. Dr. Sigrid Hoyer-Fender

(Institute for Zoology and Anthropology, Georg-August-University Göttingen)

3. Prof. Dr. Ernst A. Wimmer

(Institute for Zoology and Anthropology, Georg-August-University Göttingen)

4. Prof. Dr. Jürgen Wienands

(Department of Cellular and Molecular Immunology, Georg-August-University Göttingen)

Date of the oral examnination: $26^{\text {th }}$ June 2013 


\section{ACKNOWLEDGEMENTS}

I would like to thank my supervisor PD. Dr. med. Michael Weig for giving me the good opportunity to work on this interesting project, his nice supervision, very good suggestion when I have a problem, English correction, and especially warm welcome in the beginning of my adaptation in the institute and also thank Dr. Oliver Bader for very close teaching, guiding, supporting and English revising. Significantly, his friendship can help me to survive until this moment and I would like to call you "brother".

I would like to thank Prof. Dr. med. Uwe Groß for always giving me a very good chance for working in this institute, supporting me to go aboard for summer school, obtaining a nice position in "young ISHAM", promoting me to write the academic books and also thank Prof. Dr. Gerhard Braus for his valuable times in all of annual report and each time always give us the very good suggestions.

I would like to thank the Ministry of Science and Technology, Thailand that gave me a scholarship and also thank the Federation of European Biochemical Societies (FEBS) and the Federation of European Microbiological Societies (FEMS) that gave me the fellowship grants for aboard conference.

I would like to thank all of Thai friends in Göttingen and others city in Germany for nice parties, nice activities and nice visitings and also thank all friends in the institute for medical microbiology for very kind help and friendship, especially, Ayu Dewi Ni Nyoman for being good friend since German language class.

Finally, I would like to express my deepest gratitude to my parents and my Thai friends for their love, support, understanding and encouragement. 


\section{TABLE OF CONTENTS}

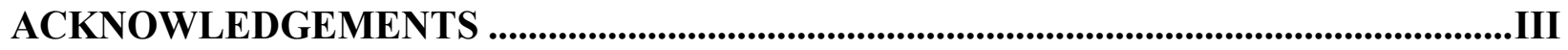

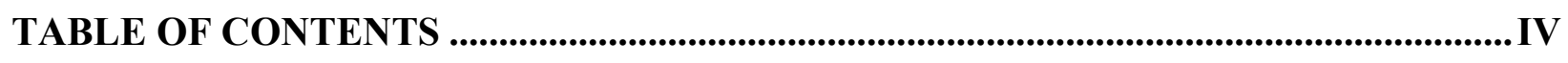

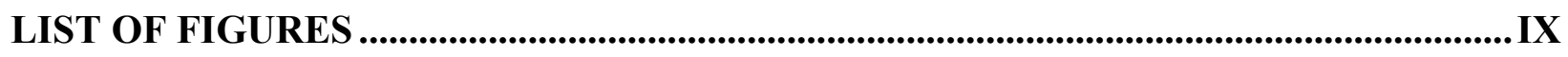

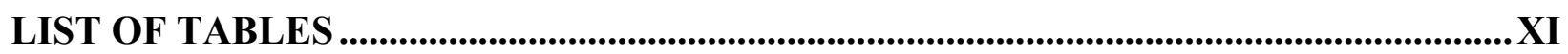

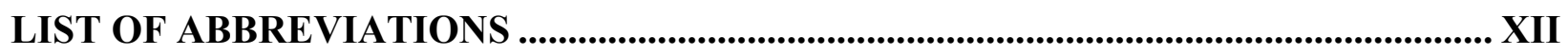

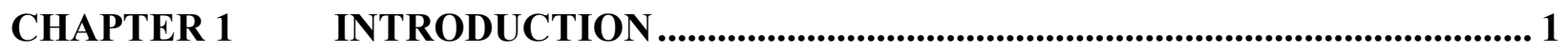

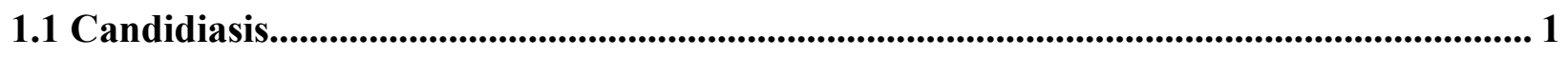

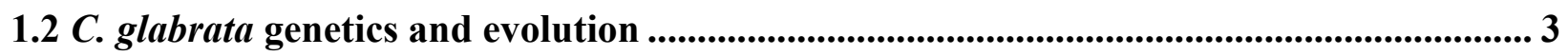

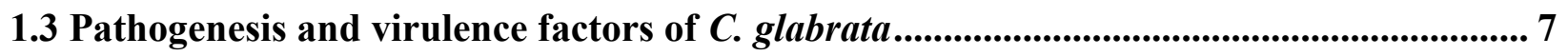

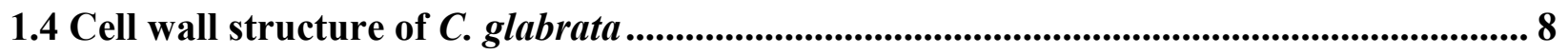

1.4.1 The polysaccharide backbone of the $C$. glabrata cell wall........................................... 8

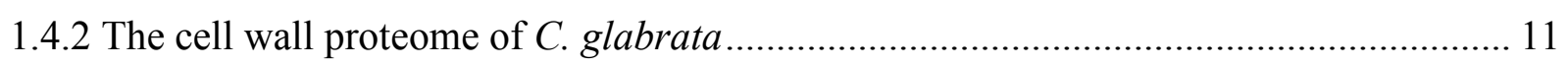

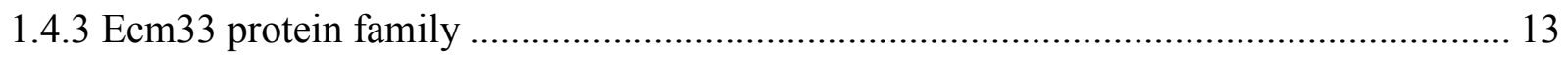

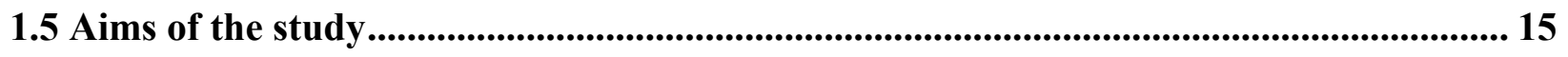

CHAPTER 2 MATERIALS AND METHODS.......................................................... 16

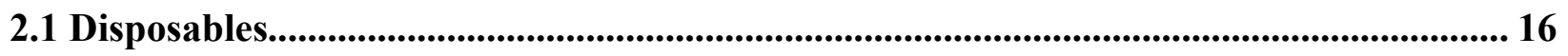

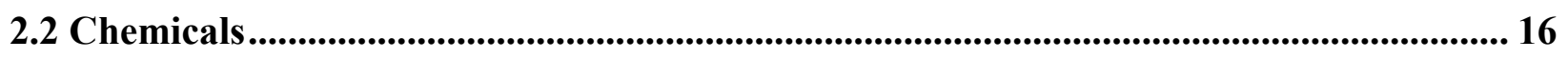




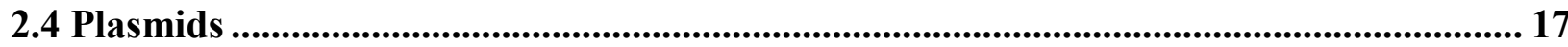

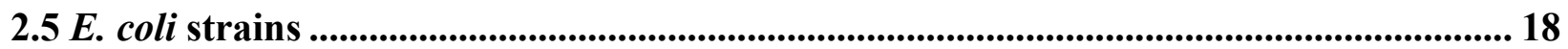

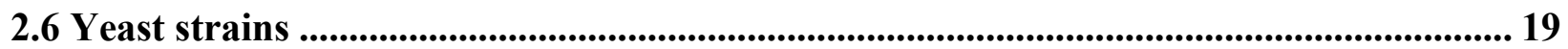

2.7 Oligonucleotides .................................................................................................................................. 21

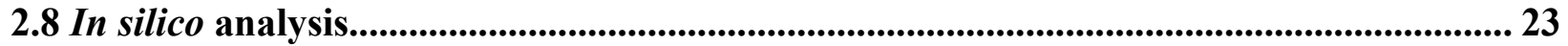

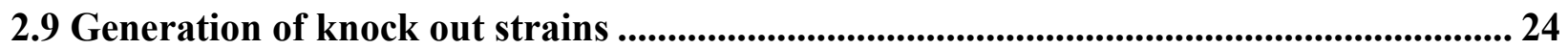

2.10 Generation of revertants and complemented strains in C. glabrata................................ 25

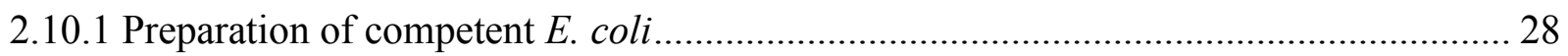

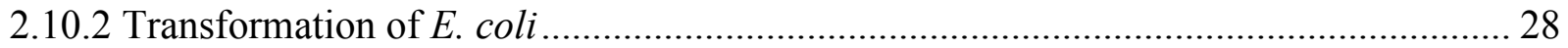

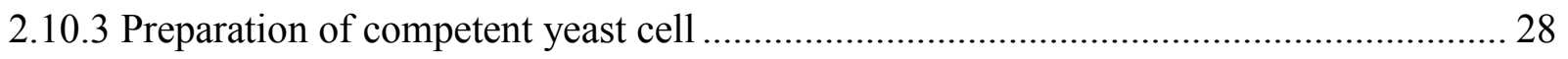

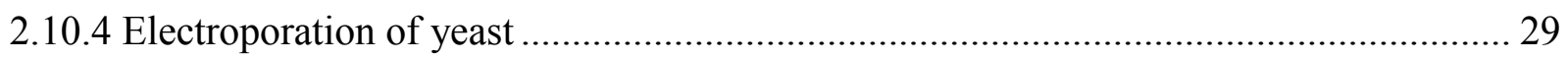

2.11 Reverse transcription real-time PCR............................................................................... 29

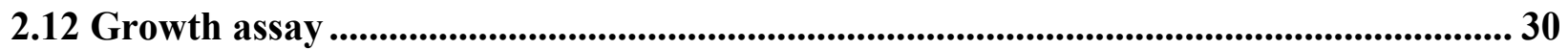

2.13 Susceptibility testing to cell perturbing agents ....................................................................... 30

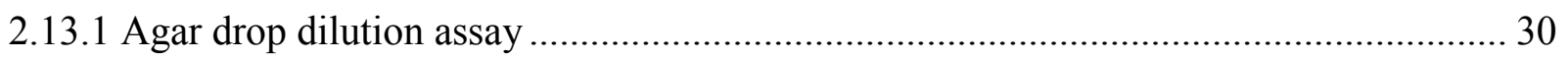

2.13.2 Broth micro-dilution assay ...................................................................................... 30

2.14 Alcian blue binding assay ..................................................................................................... 31

2.15 Flow cytometry measurement................................................................................................... 31

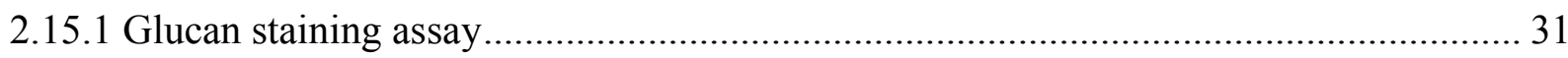

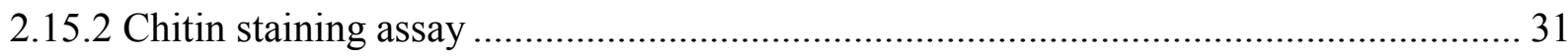

2.16 Biofilm properties determination .......................................................................................... 32

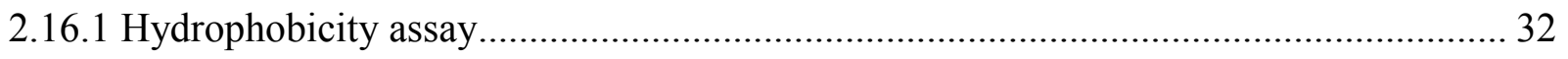




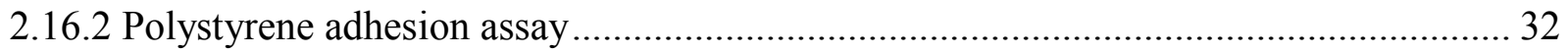

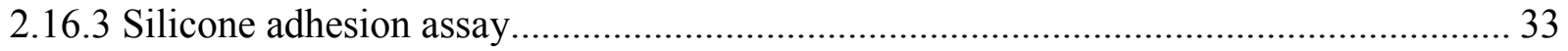

2.17 Protein expression in P. pastoris ................................................................................................. 33

2.17.1 Methanol induced Ecm33 and Pst1 protein expression ................................................. 34

2.17.2 SDS-Polyacrylamide gel electrophoresis.................................................................... 34

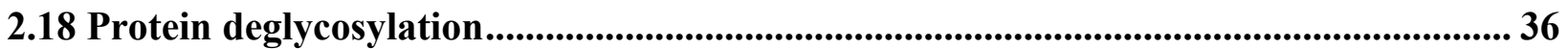

2.19 Protein expression in E. coli and Anti-Ecm33 antibody production................................ 37

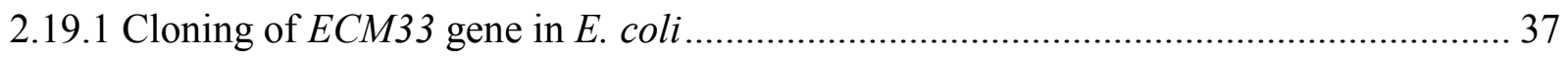

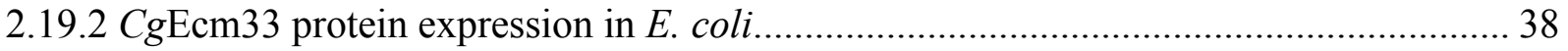

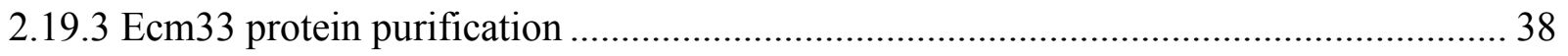

2.19.4 Protein concentration measurement ......................................................................... 38

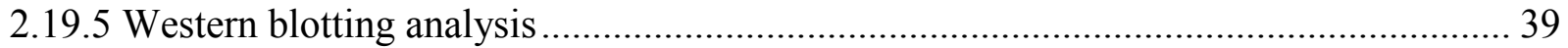

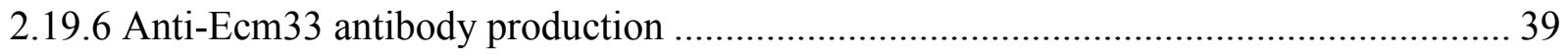

2.20 Cell wall protein release assay f......................................................................................... 40

2.21 Cell wall extraction ............................................................................................................... 40

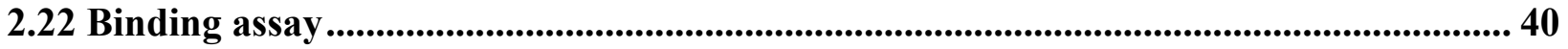

2.23 Statistical analyses ................................................................................................................... 41

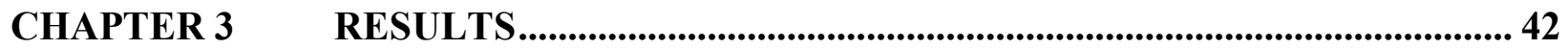

3.1 In silico analysis of the Ecm33 family ....................................................................................... 42

3.2 Confirmation of knock out strains ......................................................................................... 45

3.3 Generation of revertants and complemented strains ............................................................. 47

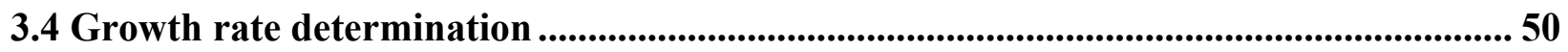

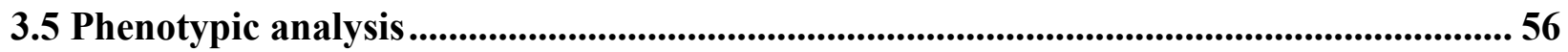




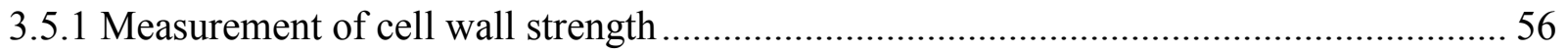

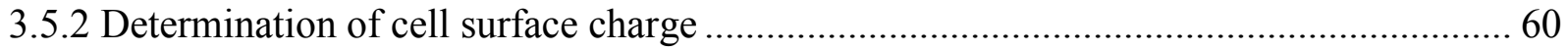

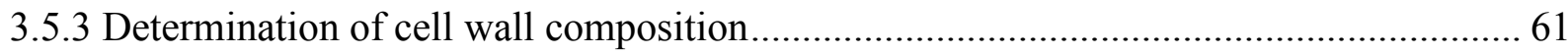

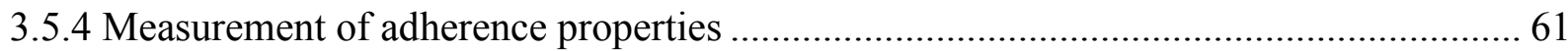

3.6 C. glabrata Ecm33 and Pst1 protein expression in P. pastoris ......................................... 65

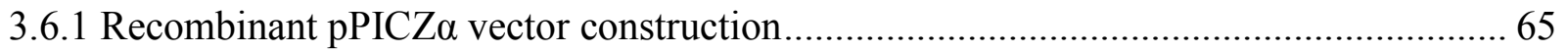

3.6.2 CgEcm33 and $C g$ Pst1 proteins expression in P. pastoris ............................................... 66

3.7 Anti-Ecm33 antibody production ............................................................................................ 68

3.7.1 Generation of plasmids for the expression of recombinant $C g E \mathrm{Em} 33$ protein in E. coli 68

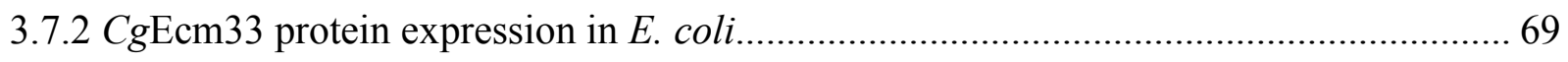

3.7.3 Anti-Ecm33 antibody production .............................................................................. 70

3.8 Cell wall protein release assay ....................................................................................................... 71

3.9 Binding assay ............................................................................................................................. 72

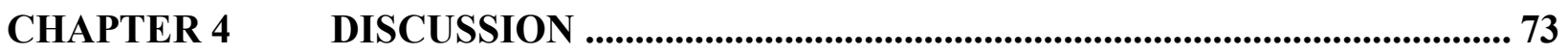

4.1 CgECM33 is required for cell fitness, cell wall integrity, and adhesion............................. 74

4.2 ECM33 of C. glabrata, C. albicans, and $S$. cerevisiae have a similar function but not

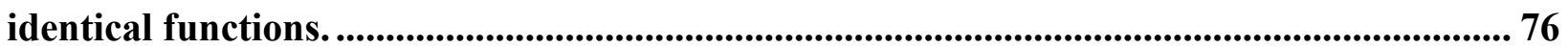

$4.3 \mathrm{Ecm} 33$ is associated with alterations in the chemical composition of the cell wall .......... 77

4.4 A model for the role of Ecm33 for the fungal cell wall.......................................................... 79

4.5 Exploring the molecular function of Ecm33 ........................................................................... 80

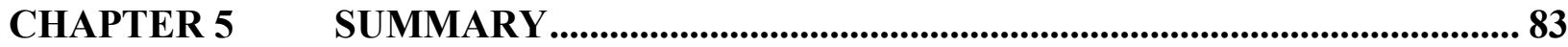

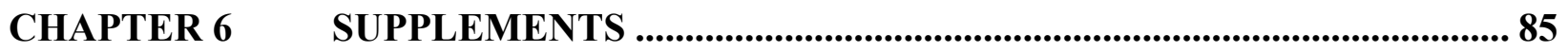

6.1 DNA sequence of $C g E C M 33$ for cloning in $E$. coli ................................................................. 85 
6.2 Process of Ecm33 and Pst1 expressed $P$. pastoris generation.

6.3 Deglycosylation of $C g E \mathrm{~cm} 33$ and $C g P s t 1$ protein and western blot analysis .................. 88

6.4 The preliminary results of competitive inhibition assay .............................................. 89

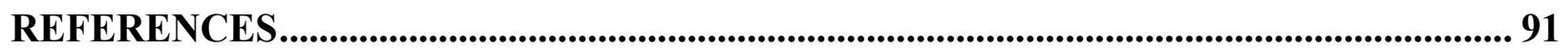

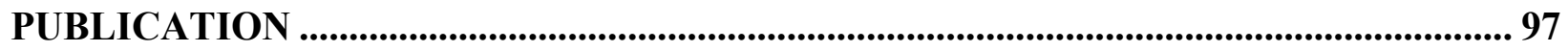

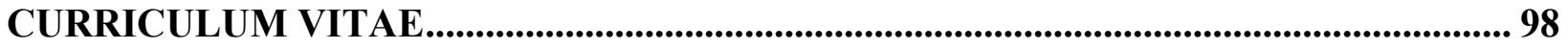




\section{LIST OF FIGURES}

Figure 1. Oral candidiasis in a 2 year old child ............................................................... 2

Figure 2. Fungal phylogenetic tree of ascomycetous taxa ................................................. 5

Figure 3. Hypothetical model of C. glabrata pathogenesis .................................................... 8

Figure 4. Structure of the yeast cell wall ............................................................................. 10

Figure 5. The sequential steps for generation of C. glabrata gene deletion cassettes................. 25

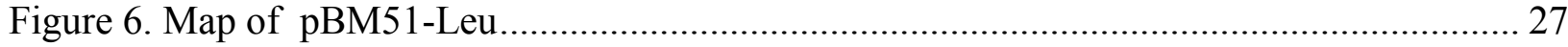

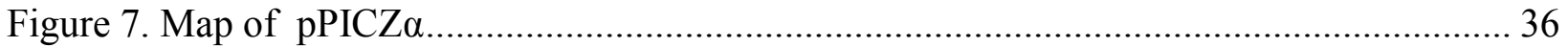

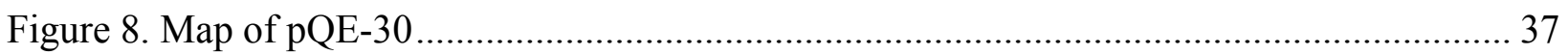

Figure 9. The time line of the anti-Ecm33 antibody production............................................ 39

Figure 10. Ecm33 protein family structure of $C$. glabrata ..................................................... 42

Figure 11. Sequence alignment of mature proteins of the Ecm33 families in C. glabrata, C.

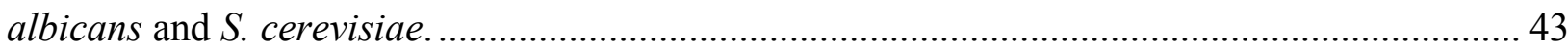

Figure 12. The Ecm33 family phylogeny of C. glabrata, C. albicans and S. cerevisiae............ 44

Figure 13. Confirmation of knock out strains by PCR ...................................................... 46

Figure 14. Reverse transcription real-time PCR to analyse the expression levels of the ECM33

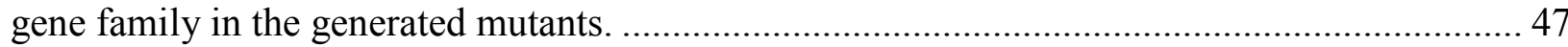

Figure 15. PCR products for recombinant pBM51 construction ............................................ 48

Figure 16. Enzyme digestion for verification of recombinant pBM51 plasmid....................... 49

Figure 17. Reverse transcription real-time PCR for the measurement of PST1 and ECM33 gene

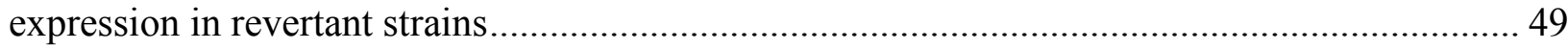

Figure 18. Expression levels of the ECM33 gene family members in complemented strains...... 50

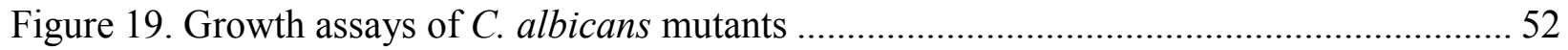

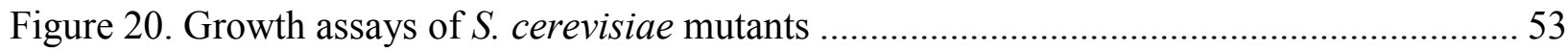

Figure 21. Growth assays of $C$. glabrata mutants ............................................................. 54

Figure 22. Growth curve of complementation experiments C. glabrata in YPD at $37^{\circ} \mathrm{C} \ldots \ldots \ldots . . .55$

Figure 23. Sensitivity testing of C. glabrata, C. albicans and S. cerevisiae to cell perturbing agents by using agar drop dilution assay ......................................................................... 57

Figure 24. Alcian blue binding of C. glabrata, C. albicans and S. cerevisiae ........................... 60 
Figure 25. FACS analysis of WGA and Congo red binding in C. glabrata, C. albicans and $S$.

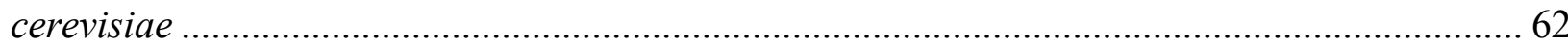

Figure 26. Adherence properties of C. glabrata, C. albicans and S. cerevisiae............................ 64

Figure 27. Amplification of $C g E C M 33$ and $C g P S T 1$ genes and verification of both recombinant

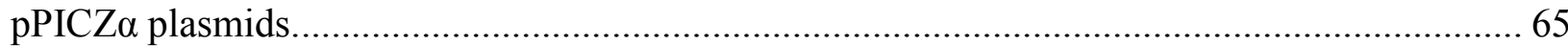

Figure 28. $\mathrm{CgEcm} 33$ and $\mathrm{CgPst} 1$ protein expression in P. pastoris ............................................. 67

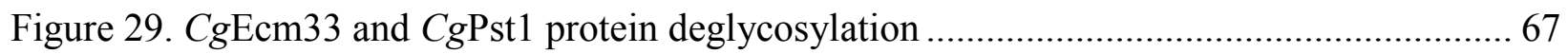

Figure 30. Amplification of the $C g E C M 33$ gene and verification of the generated

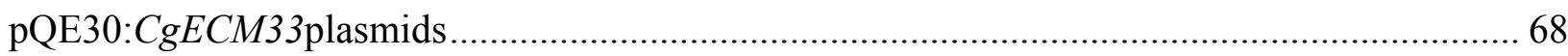

Figure 31. Purification of recombinant $C g E \mathrm{Ecm} 33$ by His-tag affinity chromatography .............. 69

Figure 32. Western blot analyses of the generated anti- $C g E c m 33$ antisera ................................. 70

Figure 33. Western blot analyses of culture supernatants.......................................................... 71

Figure 34. Binding assay.............................................................................................. 72

Figure 35. Hypothetical model of Ecm33 contribution to cell wall structure ............................... 80

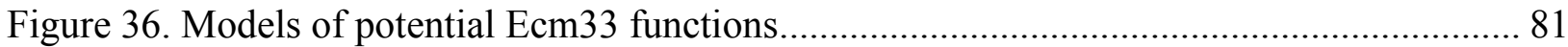

Figure 37. An overview process of Ecm 33 and Pst1 expressed P. pastoris generation ................ 87

Figure 38. Deglycosylation of $C g E c m 33$ and $C g$ Pst1 proteins and Western blot analysis .......... 88

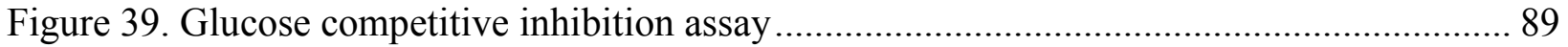

Figure 40. N-acetylglucosamine competitive inhibition assay ................................................... 90 


\section{LIST OF TABLES}

Table 1. In vitro susceptibilities of antifungal drugs ......................................................... 3

Table 2. Comparison of $C$. glabrata, $C$. albicans and $S$. cerevisiae ........................................ 6

Table 3. Cell wall composition of C. glabrata in comparison to S. cerevisiae and C. albicans . 10

Table 4. The effects of ECM33 deletion in S. cerevisiae, C. albicans, and A. fumigatus ............ 14

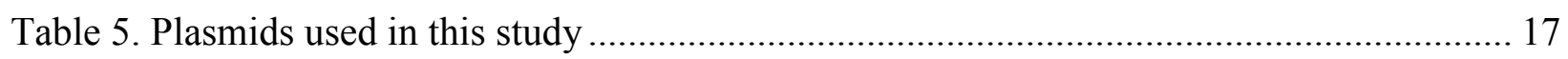

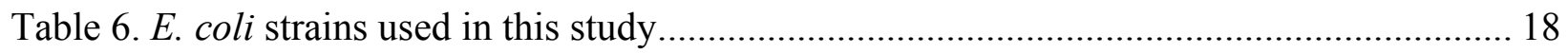

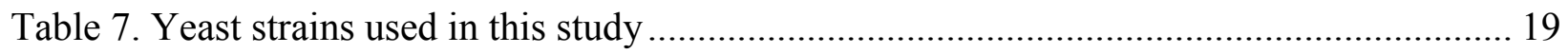

Table 8. Oligonucleotides used in this study ..................................................................... 21

Table 9. Generation of revertants and complemented strains in C. glabrata ........................... 27

Table 10. Generation of recombinant ECM33 and PST1 plasmids for protein expression .......... 35

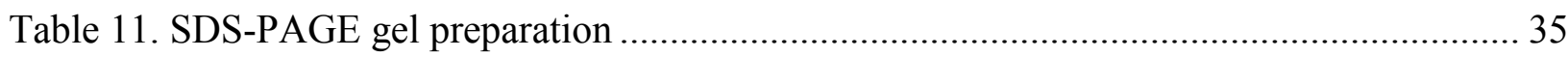

Table 12. Identity matrix of the entire protein in the Ecm33 family of C. glabrata, C. albicans

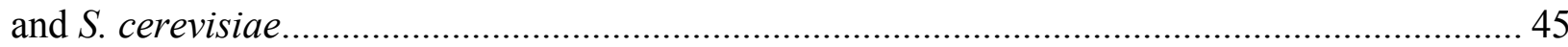

Table 13. Identity matrix of Ecm33 family effector domains only of C. glabrata, C. albicans and

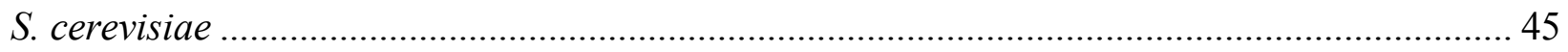

Table 14. Summary of results obtained by broth microdilution assay ..................................... 59 


\section{LIST OF ABBREVIATIONS}

\begin{tabular}{|c|c|}
\hline AIDS & Acquired immunodeficiency syndrome \\
\hline APS & Ammonium persulfate \\
\hline BLAST & Basic local alignment search tool \\
\hline $\mathrm{BMMH}$ & Buffered minimal methanol \\
\hline BSA & Bovine serum albumin \\
\hline cDNA & Complementary DNA \\
\hline CFW & Calcoflour white \\
\hline $\mathrm{ddH}_{2} \mathrm{O}$ & Double distilled water \\
\hline DMSO & Dimethyl sulfoxide \\
\hline DNA & Deoxyribonucleic acid \\
\hline dNTP & Dinucleotide phosphate \\
\hline DTT & 1,4-Dithiothreitol \\
\hline $\mathrm{ECL}$ & Enhanced chemiluminescent detection \\
\hline ECWs & Extracted cell walls \\
\hline EDTA & Ethylene diamine tetraacetic acid \\
\hline FACS & Fluorescence activated cell sorting \\
\hline $\mathrm{g}$ & Force of gravity \\
\hline IPTG & Isopropyl beta-D-thiogalactopyranoside \\
\hline $\mathrm{kb}$ & Kilo base pair \\
\hline $\mathrm{kDa}$ & Kilo Dalton \\
\hline LB & Luria-Bertani medium \\
\hline M & Molar \\
\hline
\end{tabular}




\begin{tabular}{|c|c|}
\hline $\mathrm{ml}$ & Milliliter \\
\hline $\min$ & Minute \\
\hline $\mathrm{mg}$ & Milligram \\
\hline$\mu \mathrm{g}$ & Microgram \\
\hline$\mu 1$ & Microliter \\
\hline$\mu \mathrm{m}$ & Micrometer \\
\hline MM & Minimal medium \\
\hline MOMP & Mitochondrial outer membrane permeabilisation \\
\hline mRNA & Messenger RNA \\
\hline OD & Optical density \\
\hline$p$ & $P$ value \\
\hline PAGE & Polyacrylamide gel electrophoresis \\
\hline PBS & Phosphate buffered saline \\
\hline PCR & Polymerase chain reaction \\
\hline RNA & Ribonucleic acid \\
\hline RNase & Ribonuclease \\
\hline rpm & Revolutions per minute \\
\hline RPMI & Roswell Park Memorial Institute \\
\hline RT & Room temperature \\
\hline RT-PCR & Reverse transcription-polymerase chain reaction \\
\hline $\mathrm{sec}$ & Second \\
\hline SDS & Sodium dodecyl sulphate \\
\hline SD & Standard deviation \\
\hline TEMED & $\mathrm{N}, \mathrm{N}, \mathrm{N}$ ', N'-tetramethylethylenediamine \\
\hline
\end{tabular}




$\begin{array}{ll}\text { tet } & \text { Tetracycline } \\ \text { Tm } & \text { Annealing temperature } \\ \text { Tris } & \text { Tris(hydroxylmethyl)-aminomethan } \\ \text { U } & \text { Unit } \\ \text { UV } & \text { Ultraviolet } \\ \text { V } & \text { Voltage } \\ \text { v } & \text { Volume } \\ \text { w } & \text { Weight } \\ \text { WT } & \text { Wild-type } \\ \text { YPD } & \text { Yeast Peptone Dextrose }\end{array}$




\section{CHAPTER 1}

\section{INTRODUCTION}

\subsection{Candidiasis}

The incidence of fungal infections in humans is increasing worldwide because of the rising numbers of immunocompromised hosts, resulting from AIDS, chemotherapy for cancer patients, and patients receiving immunosuppressive drugs for transplantation and auto-immune diseases. Clinical manifestations of fungal infections may be relatively harmless (e.g. in the case of dermatophytes or Malassezia species), but invasive fungal infections (IFIs) can be lifethreatening (Giri, S. and Kindo, A. J., 2012; Sifuentes-Osornio, J., Corzo-Leon, D. E. et al., 2012; Mayer, F. L., Wilson, D. et al., 2013).

Candida species, Aspergillus species and Cryptococcus neoformans are three main organisms causing invasive fungal infections in humans (Liao, Y., Chen, M. et al., 2013). Candida species are considered as normal flora which can be found in the oral cavity and the gastrointestinal tract of most healthy humans (Fidel, P. L., Jr., Vazquez, J. A. et al., 1999; Brunke, S. and Hube, B., 2012) and the majority of Candida isolates from clinical samples represent asymptomatic colonization (Brunke, S. and Hube, B., 2012). However, when the human body is weakened by prolonged treatment with antibiotics, diabetes, cancer, extreme age, immunosuppression, intravenous catheters or long-term hospitalization, these fungi can cause a broad range of clinical manifestations ranging from local superficial to life-threatening systemic infections, with high morbidity and mortality (Perlroth, J., Choi, B. et al., 2007; Brunke, S. and Hube, B., 2012) (Figure 1). Infections caused by Candida species are termed "candidiasis". More than $90 \%$ of candidiasis cases are attributed to only five Candida species: C. albicans, $C$. glabrata, C. parapsilosis, C. tropicalis and C. krusei. Nevertheless, rare species of Candida are isolated from candidiasis patients every year such as $C$. guilliermondii, $C$. inconspicua, $C$. rugosa, and C. lambica (Pfaller, M. A., Diekema, D. J. et al., 2010; Giri, S. and Kindo, A. J., 2012). C. albicans is the most prevalent species in both systemic and superficial infections 
worldwide. The second most prevalent species depends on the site of infection and geography. For example, in the United States, Europe, and Asia-Pacific, C. glabrata is considered the second most prevalent species (Marchetti, O., Bille, J. et al., 2004; Borg-von Zepelin, M., Kunz, L. et al., 2007; Pfaller, M. A., Diekema, D. J. et al., 2010), but in Latin America (e.g. Brazil), $C$. tropicalis and C. parapsilosis are the most important species after C. albicans (Colombo, A. L., Guimaraes, T. et al., 2007).

In contrast to $C$. albicans, C. glabrata infections are very difficult to treat, as this organism is often highly resistant to azole antifungals. Amphotericin B and echinocandins are considered as effective drugs to eliminate this pathogenic yeast from the patients, but Amphotericin B has a lot of side effects and echinocandins is still very expensive. $C$. parapsilosis is less susceptible to echinocandins and C. krusei is fully resistant to Fluconazole (Table 1). Therefore, infections due to these non-albicans species are causes of increased morbidity and mortality (Marchetti, O., Bille, J. et al., 2004). Consequently, species identification in Candidiasis is essential to enable early and adequate antifungal therapy.

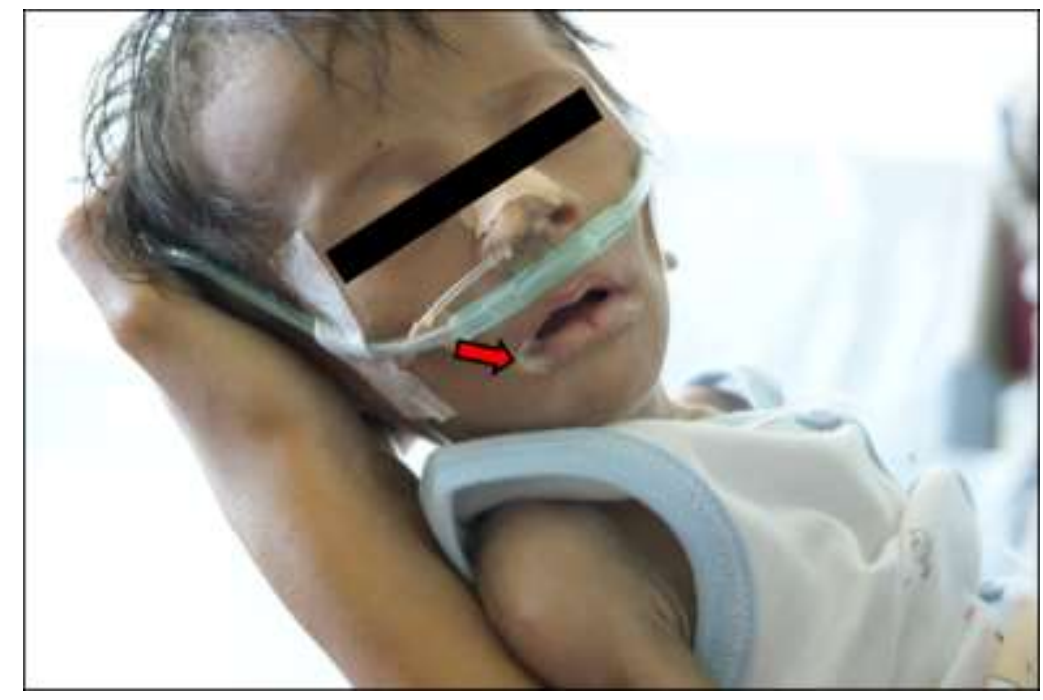

Figure 1. Oral candidiasis in a 2 year old child

The child suffers from acquired immunodeficiency syndrome (AIDS) after infection with human immunodeficiency virus (HIV) by its mother.

(Taken by Tangwattanachuleeporn M., 2011 in Makassar, Indonesia) 
Table 1. In vitro susceptibilities of antifungal drugs

\begin{tabular}{|c|c|c|c|c|c|c|}
\hline & C. albicans & C. glabrata & C. tropicalis & C. parapsilosis & C. krusei & other \\
\hline Fluconazole & + & $+/-$ & + & + & - & + \\
\hline Itraconazole & + & $+/-$ & + & $+/-$ & $+/-$ & + \\
\hline Posaconazole & + & $+/-$ & + & + & + & + \\
\hline Voriconazole & + & $+/-$ & + & + & + & + \\
\hline Ketoconazole & + & + & + & + & + & + \\
\hline Amphotericin B & + & + & + & + & $+/-$ & + \\
\hline Flucytosine & $+/-$ & + & $+/-$ & + & $+/-$ & $+/-$ \\
\hline Nystatin & + & + & + & + & + & + \\
\hline Echinocandins $^{\mathrm{a}}$ & + & + & + & - & + & + \\
\hline
\end{tabular}

This data were combinated from (Richter, S. S., Galask, R. P. et al., 2005; Leventakos, K., Lewis, R. E. et al., 2010)

+ : in vitro activity, -: no in vitro activity, +/-: modest in vitro activity

a: Caspofungin, micafungin, and anidulafungin

\subsection{C. glabrata genetics and evolution}

In the past, C. glabrata was classified in the genus of Torulopsis and termed Torulopsis glabrata because it was thought to be unable to form non-hyphae. Subsequently, when conditions for pseudohypha production in T. glabrata were discovered, the organism was reclassified in the genus Candida and renamed C. glabrata (Fidel, P. L., Jr., Vazquez, J. A. et al., 1999).

C. glabrata belongs to the class Saccharomycetes in the order Saccharomycetales and the family Saccharomycetaceae. The yeast cells of $C$. glabrata are 1 to $4 \mu \mathrm{m}$ in size and smaller than the $C$. albicans yeast form (4 to $6 \mu \mathrm{m})$. C. glabrata cannot produce pseudohyphae under normal 
condition but it can produce pseudohyphae on solid synthetic low ammonia dextrose nitrogen starvation medium (SLAD) after incubation at $37^{\circ} \mathrm{C}$ for 2 weeks (Csank, C. and Haynes, K., 2000). Colonies of C. glabrata on Sabouraud dextrose agar are smooth, cream colored and glistening. Like $S$. cerevisiae, C. glabrata is haploid genome yeast. In contrast, C. albicans and several other non-albicans Candida species are diploid (Fidel, P. L., Jr., Vazquez, J. A. et al., 1999).

The genome of the reference strain C. glabrata CBS138 (originally isolated from human feces) has been sequenced and it shows 13 chromosomes totalling in $12.3 \mathrm{Mb}$. There are approximately 5,283 coding sequences (CDS), 207 tRNA genes and the genome has a $\mathrm{G}+\mathrm{C}$ content of 38.8\% (http://genolevures.org/cagl.html and (Kaur, R., Domergue, R. et al., 2005)).

The results from phylogenetic analyses show that $C$. glabrata is quite distinct from $C$. albicans and the other pathogenic Candida species, but it is closely related to $S$. cerevisiae (Figure 2) (Diezmann, S., Cox, C. J. et al., 2004; Kaur, R., Domergue, R. et al., 2005). C. glabrata shares a common ancestor to $S$. cerevisiae and clearly belongs to a clade different from that of other Candida species. As a consequence, the CUG codon of C. glabrata and $S$. cerevisiae is translated to leucine, but in other Candida species it is translated to Serine (Roetzer, A., Gabaldon, T. et al., 2011). Many genes of $S$. cerevisiae have orthologues in C. glabrata and the gene orders are largely conserved between both organisms (Roetzer, A., Gabaldon, T. et al., 2011). However, C. glabrata displays a significantly higher degree of gene loss, resulting in a regressive evolution (loss of specific functions) as compared to $S$. cerevisiae (Dujon, B., Sherman, D. et al., 2004). Indeed, C. glabrata lacks the genes that are involved in galactose and sucrose assimilation, phosphate, nitrogen, and sulfur metabolism and chemical biosynthesis of nicotinic acid, thiamine and pyridoxine (Kaur, R., Domergue, R. et al., 2005).

The adhesin genes of C. glabrata (e.g. Epa protein family) and S. cerevisiae (Flo protein family) locate in subtelomeric regions of the chromosomes. Since both adhesin families share some degree of functional homology and the tandem repeat motifs of several Epa and Flo proteins are similar (Roche, 2007). Thus, it is assumed that the FLO and EPA genes are evolutionarily related but their sequences have diverged beyond recognition. However, Epa adhesin from C. glabrata may have occured from adaptation because of the survival within mammalian hosts (Roetzer, A., Gabaldon, T. et al., 2011). 


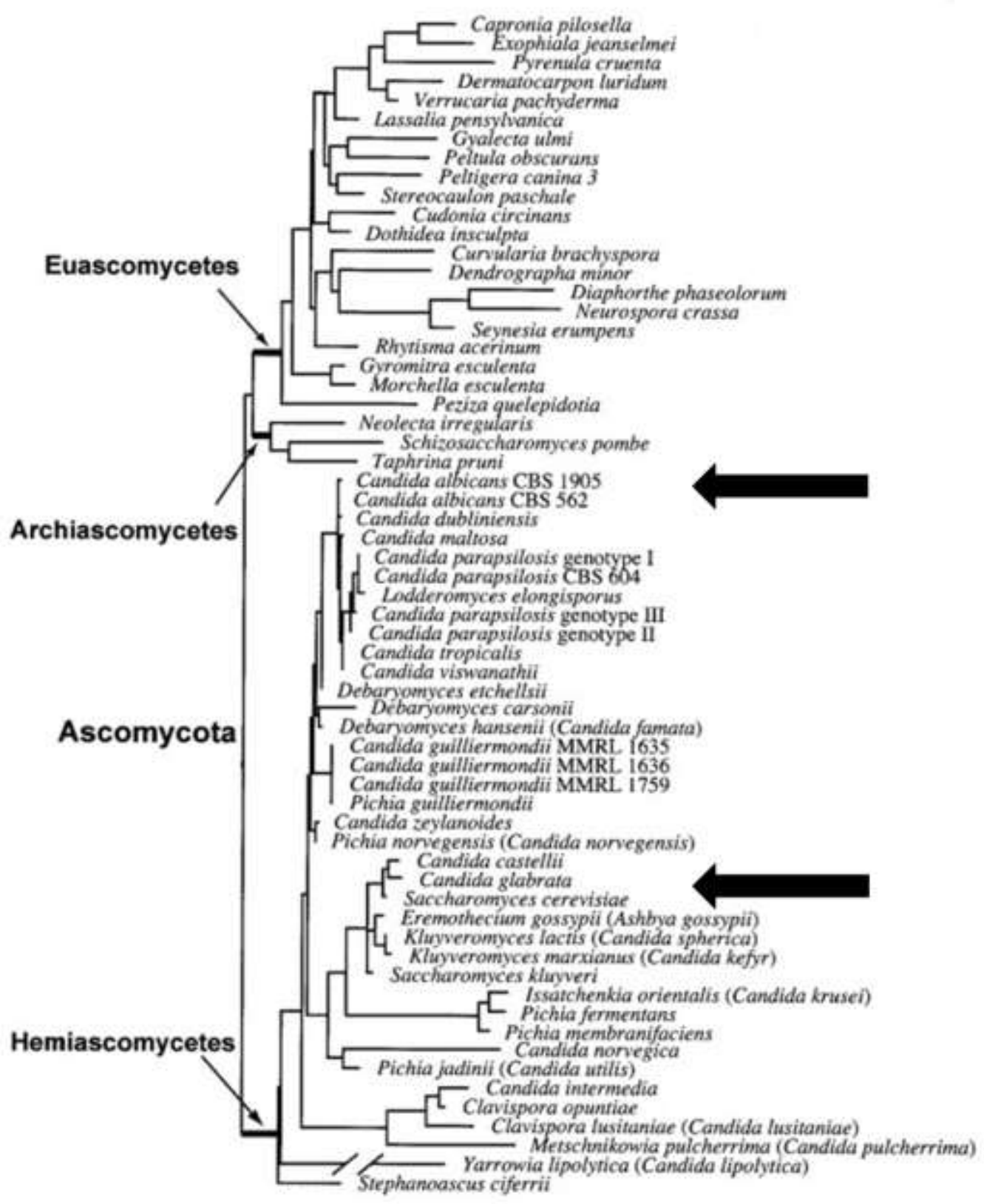

-0.01 substitutions/site

\section{Figure 2. Fungal phylogenetic tree of ascomycetous taxa}

Phylogenetic tree based on a combined analysis of the nuclear 18S rDNA and 26 rDNA of ascomycetous taxa. The nodes marking the Euascomycetes, Archiascomycetes, and Hemiascomycetes as well as many terminal branches are supported by homogeneous Bayesian posterior probabilities $95 \%$. Black arrows indicate the origins of the three classes of the phylum Ascomycota. Red arrows indicate C. albicans and C. glabrata and S. cerevisiae (modified from the article of (Diezmann, S., Cox, C. J. et al., 2004)). 
Table 2. Comparison of $C$. glabrata, $C$. albicans and $S$. cerevisiae

\begin{tabular}{|c|c|c|c|}
\hline & C. glabrata & C. albicans & S. cerevisiae \\
\hline $\begin{array}{l}\text { Number of } \\
\text { chromosomes }\end{array}$ & 13 chromosomes & 8 chromosomes & 16 chromosomes \\
\hline Length of DNA & $12.3 \mathrm{Mb}$ & $14.3 \mathrm{Mb}$ & $12.1 \mathrm{Mb}$ \\
\hline Coding genes & 5,283 & 6,202 & 5,807 \\
\hline Ploidy & Haploid (n) & Diploid (2n) & Diploid (2n) \\
\hline CUG codon & Leucine & Serine & Leucine \\
\hline Clade & WGD & CTG & WGD \\
\hline Virulence & $\begin{array}{l}\text { Opportunistic } \\
\text { pathogen }\end{array}$ & $\begin{array}{l}\text { Opportunistic } \\
\text { pathogen }\end{array}$ & Non-pathogenic \\
\hline Major sites of infection & $\begin{array}{l}\text { Oral, vaginal, } \\
\text { disseminated }\end{array}$ & $\begin{array}{l}\text { Oral, vaginal, } \\
\text { disseminated }\end{array}$ & Non-infectious \\
\hline Mating genes & Present & Present & Present \\
\hline Sexual cycle & Unknown & Known & Known \\
\hline $\begin{array}{l}\text { Clonal population } \\
\text { structure }\end{array}$ & Yes & Yes & No \\
\hline Phenotypic switching & Present & Present & Absent \\
\hline True hyphae & Absent & Present & Absent \\
\hline Pseudohyphae & Present & Present & Present \\
\hline Biofilm formation & Present & Present & Present \\
\hline Major adhesins & Epa family & $\begin{array}{l}\text { Hwp1 and Als } \\
\text { family }\end{array}$ & Flo family \\
\hline Auxotrophy & $\begin{array}{l}\text { Niacin, thiamine, } \\
\text { pyridoxine }\end{array}$ & None & None \\
\hline Azole resistance & Innate resistance & Susceptible & Susceptible \\
\hline Mitochondrial function & Petite positive & Petite negative & Petite positive \\
\hline
\end{tabular}

This table was modified from (Kaur, R., Domergue, R. et al., 2005) 


\subsection{Pathogenesis and virulence factors of $C$. glabrata}

Normally, C. glabrata is a commensal microorganism in the human body, but when the host's immune system is compromised, it can also cause severe infections. On mucosal surfaces, C. glabrata colonizes by expressing specific adherence-genes such as the CgEPA family, $C g \underline{S}$ ilent Information $\underline{\text { Regulator }}(S I R 3)$, and $C g$ Pyrazinamidase and NiCotinamidase (PNC1) enabling it to form biofilms. C. glabrata also has an evasive strategy to manage phagocytosis by macrophages. After uptake, macrophages initially induce formation of peroxisomes. These peroxisomes are degraded by $C$. glabrata using the pexophagy pathway, a specific subtype of autophagy. The autophagy related $C g \underline{A}$ uTophaGy $(A T G)$ gene family contributes to the survival of $C$. glabrata during engulfment and this process requires mobilization of resources to survive in phagosome. C. glabrata is relative resistant to carbon starvation (Roetzer, A., Gabaldon, T. et al., 2011). Carbon starvation of engulfed C. glabrata is associated with the up-regulation of $C g \underline{I}$ so $\underline{\text { itrate }}$ Lyase $(I C L 1)$ and $C g \underline{\text { MaLate }} \underline{\text { Synthase }}(M L S 1)$ genes in the glyoxylate cycle (Fukuda, Y., Tsai, H. F. et al., 2013). The oxidative burst response in phagosomes of macrophages is administrated by neutralization of the oxidative reaction and increase the $\mathrm{pH}$ in phagosome for the survival of C. glabrata. Both reactions contribute to activation of CgY Yeast AP (YAPl), a transcription factor responsive to $\mathrm{H}_{2} \mathrm{O}_{2}$ and $C g \underline{S}$ uperoxide Dismutases (SOD1). Fequently, $C$. glabrata strains are resistant to antifungal drugs, especially fluconazole, mediated through efflux pumps $C g P D R 1$ and $C g C D R 1$ (Ferrari, S., Sanguinetti, M. et al., 2011; Roetzer, A., Gabaldon, T. et al., 2011; Orta-Zavalza, E., Guerrero-Serrano, G. et al., 2013) as shown in Figure 3. 


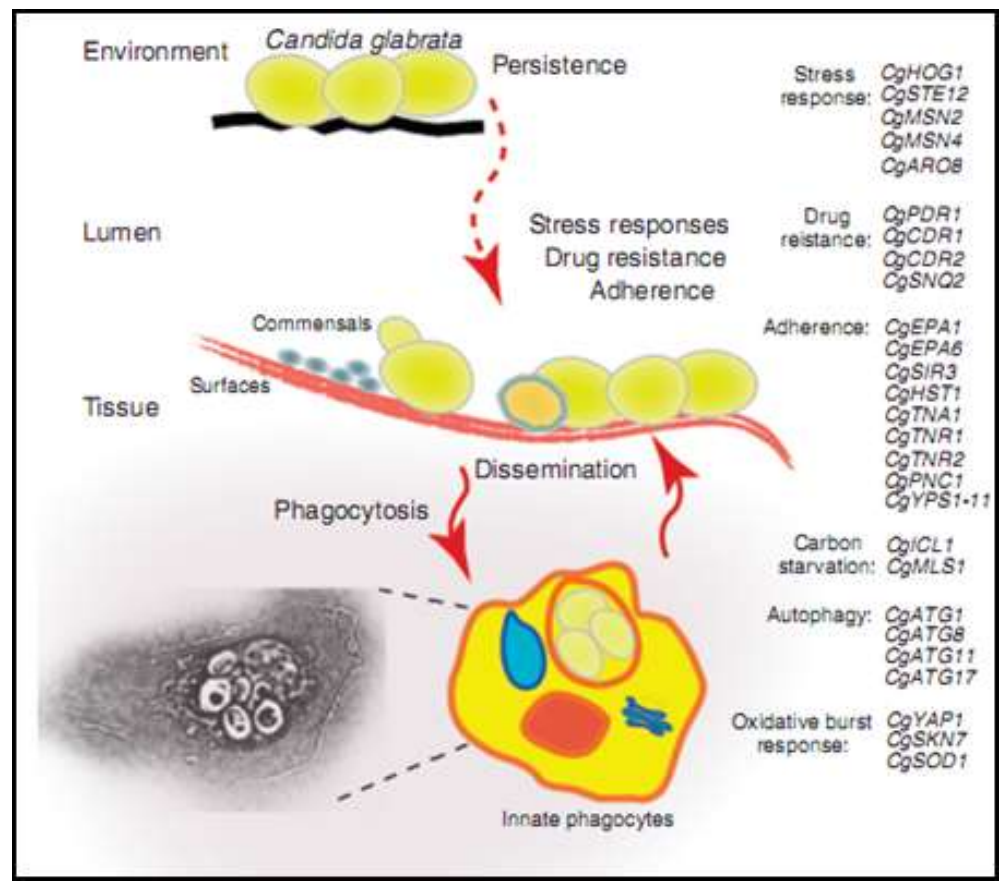

Figure 3. Hypothetical model of $C$. glabrata pathogenesis

C. glabrata is found as normal flora in mucosal areas of humans, such as the gut. In immunocompromised or elderly persons, C. glabrata is able to spread into tissues and cause organ failure. The control of phagocytes is necessary for elimination of the invading C. glabrata. The right panel shows a list of genes that contribute to each stage of C. glabrata infection (Figure taken from (Roetzer, A., Gabaldon, T. et al., 2011)).

\subsection{Cell wall structure of $C$. glabrata}

\subsubsection{The polysaccharide backbone of the $C$. glabrata cell wall}

The cell wall of fungi is one of the most important organelle. It determines cell shape, physical strength and limits permeability. Furthermore, it contributes to adherence to host cells or medical materials and to biofilm formation. The cell wall can be considered a "virulence associated factor", since it determines the initial host - fungus interactions leading to mycoses. Moreover, the cell wall triggers the human immune response, especially the innate immunity and it is also connect to antifungal drug resistance (Weig, M., Jansch, L. et al., 2004; de Groot, P. W., Kraneveld, E. A. et al., 2008). 
Generally, the fungal cell wall consists of $80-90 \%$ polysaccharides, mainly glucans and mannans. In yeast cell wall, the outer layer cell wall is highly enriched with O- and N-linked mannans that are covalently associated with proteins to form glycoproteins, whereas the inner layer contains the skeletal polysaccharides chitin and $\beta-1,3$-glucan, which confer strength and cell shape. The outer cell wall proteins are attached to this inner wall framework predominantly by glycosylphosphatidylinositol (GPI) remnants that are linked to the skeleton through a more flexible $\beta$-1,6-glucan. The cell wall of molds, e.g. Aspergillus fumigatus, it is composed of a unique $\beta-1,3 / 1,4$-glucan skeleton with chitin and galactomannan covalently linked to the nonreducing ends of $\beta$-1,3-glucan. The cell wall is mainly coated with GPI proteins, which contain N- and O-glycans (Jin, C., 2012). In comparison, the cell walls of molds have a higher complexity of carbohydrates and chitin components than yeast.

The cell wall of $C$. glabrata contains approximately $54 \%$ glucans, $43.8 \%$ mannans, $1.2 \%$ chitin, and $6.4 \%$ proteins (Table 3 ) and the architecture of the cell wall in C. glabrata is shown in Figure 4.

In summary, the cell wall of $C$. glabrata displays a high level of proteins and mannans, but a low level of chitin and glucan, including 1,6- $\beta$-glucan and 1,3- $\beta$-glucan, when compared with S. cerevisiae and C. albicans (de Groot, P. W., Kraneveld, E. A. et al., 2008). Therefore, it is assumed that cell wall of $C$. glabrata has more mannoproteins on its outer surface than others yeasts. 

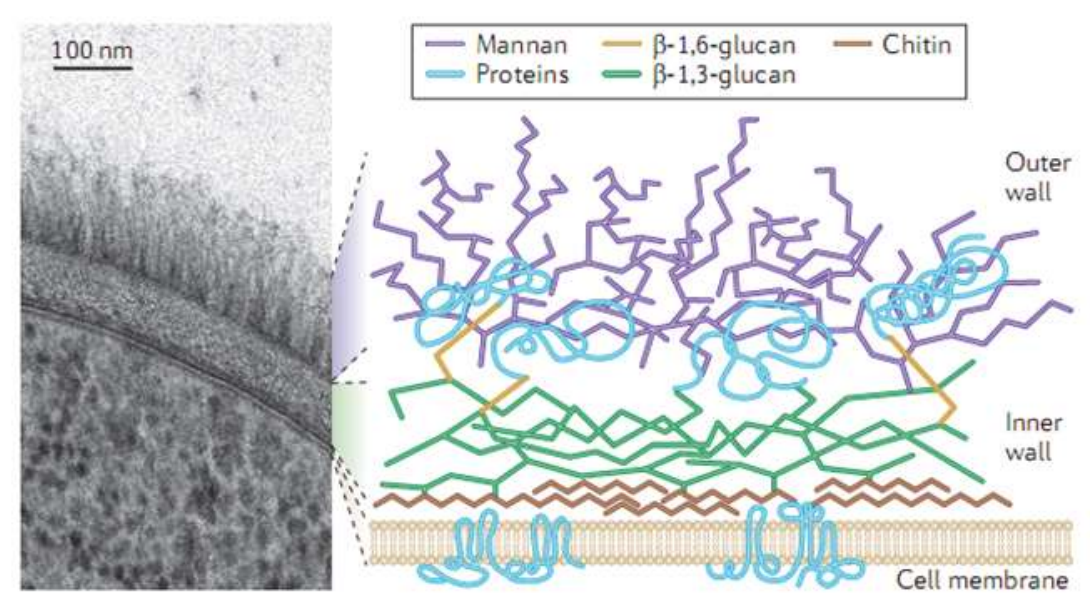

Figure 4. Structure of the yeast cell wall

The outer layer of the cell wall is highly enriched with $\mathrm{O}$ - and N-linked mannose polymers (mannans) that are covalently associated with proteins to form glycoproteins, whereas the inner layer contains the skeletal polysaccharides chitin and $\beta$-1,3-glucan, which confer strength and cell shape. The outer cell wall proteins are attached to this inner wall framework predominantly by glycosylphosphatidylinositol (GPI) remnants that are linked to the skeleton through a more flexible $\beta$-1,6-glucan. Figure was taken from (Gow, N. A., van de Veerdonk, F. L. et al., 2012).

Table 3. Cell wall composition of $C$. glabrata in comparison to $S$. cerevisiae and $C$. albicans

\begin{tabular}{lccccccc}
\hline Organisms & \multicolumn{9}{c}{ Cell wall content } \\
\cline { 2 - 7 } & Protein (\%) & Chitin (\%) & Man (\%) & Glu (\%) & M/G & \multicolumn{2}{c}{ Alkali-insoluble glucan } \\
\cline { 3 - 7 } & & & & & $1,6)$ & $1,3-\beta$ \\
\hline C. glabrata & $6.4 \pm 0.1$ & $1.2 \pm 0.1$ & $43.8 \pm 0.5$ & $54.0 \pm 0.2$ & 0.81 & $4.2 \pm 0.1$ & $16.7 \pm 1.7$ \\
S. cerevisiae & $4.0 \pm 0.1$ & $1.4 \pm 0.2$ & $34.2 \pm 1.6$ & $60.3 \pm 2.5$ & 0.57 & $7.1 \pm 0.2$ & $26.8 \pm 0.9$ \\
C. albicans & $3.5 \pm 0.2$ & $4.2 \pm 0.1$ & $26.6 \pm 2.3$ & $64.0 \pm 4.9$ & 0.42 & $10.6 \pm 0.6$ & $26.2 \pm 1.1$ \\
\hline
\end{tabular}

Cell wall content values are from exponentially growing cells (in YPD) and are expressed as percentages of freeze-dried cell walls. Man: mannose, Glu: glucose, M/G: ratio of mannose to glucose. Table taken from (de Groot, P. W., Kraneveld, E. A. et al., 2008). 


\subsubsection{The cell wall proteome of $C$. glabrata}

Covalently linked wall protein of C. glabrata can be divided 2 groups as (i) GPI cell wall proteins, which can be specifically released with HF-pyridine and (ii) mild alkali-soluble cell wall proteins, which can be released by mild $\mathrm{NaOH}$ (Klis, F. M., Brul, S. et al., 2010). The majority of cell wall proteins are linked to the carbohtdrate complex via a covalent bond of a sugar remnant of their GPI anchor and 1,6- $\beta$-glucan. The minority of cell wall proteins are attached to 1,3- $\beta$-glucan through a linkage which sensitive to mild alkali. The covalently linked wall proteins have a variety of function as (i) carbohydrate-active enzyme such as Crh1, Scw4, and Gas family, (ii) other enzymatic activity such as Plb2, (iii) nonenzymatic cell wall proteins such as Cwp family, Pir family, and Ssr1, and (iv) adhesin such as Epa family, and Awp family (de Groot, P. W., Kraneveld, E. A. et al., 2008).

The most abundant covalently linked wall proteins of $C$. glabrata were identified by

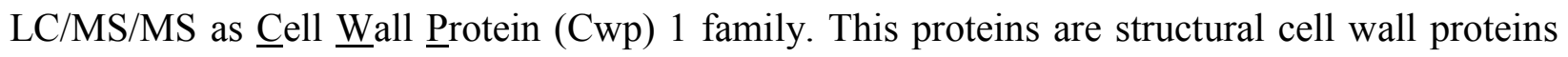
that connected to the cell wall either through the GPI anchor and alkali sensitive linkage (Schmidt, P., 2007; de Groot, P. W., Kraneveld, E. A. et al., 2008).

In silico analysis in C. glabrata revealed that there are 106 glycosylphosphatidylinositolmodified (GPI) proteins in the cell wall and 50\% of these GPI proteins have features of adhesin-

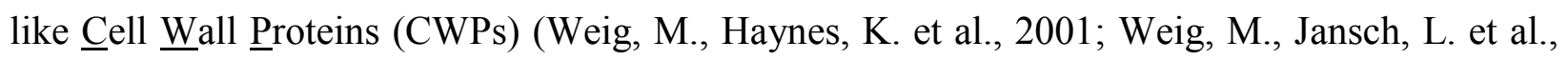
2004; de Groot, P. W., Kraneveld, E. A. et al., 2008)

One of the noticeable characteristics of Candida spp. is their ability to firmly adhere to host surface cells, including the human skin, endothelial, and epithelial mucosal host tissues. Adhesion is considered an important first step of pathogenesis in fungal infections. Candida spp. also attach to medical abiotic surfaces such as intravascular and urinary catheters, prosthetic cardiac valves, and denture prostheses (Busscher, H. J., Rinastiti, M. et al., 2010; de Groot, P. W., Bader, O. et al., 2013). Most of known fungal adhesins are large (usally more than 800 amino acids) GPI-modified wall proteins. In silico analyses showed that C. glabrata has significantly more potential adhesin genes than $C$. albicans and $S$. cerevisiae. Two-thirds of these 66 putative adhesin genes in C. glabrata are situated in regions of subtelomeres. In all subtelomeric parts of reference strain CBS138 at least one of putative adhesin gene is present (de Groot, P. W., Kraneveld, E. A. et al., 2008; de Groot, P. W., Bader, O. et al., 2013). 
A well-known adhesin family of C. glabrata is the Epa (epithelial adhesion protein) family with 17 members in reference strain CBS138. The reference strain BG2 has two additional EPA genes EPA4 and EPA5 (Kaur, R., Domergue, R. et al., 2005; de Groot, P. W., Bader, O. et al., 2013). Additionally, none of the orthologous EPA genes in either strain are $100 \%$ identical. The $E P A$ family is associated with adhesion to human epithelial and endothelial cells, particularly EPA1, EPA6 and EPA7 (Domergue, R., Castano, I. et al., 2005; Kaur, R., Domergue, R. et al., 2005). Data from Bader et al. revealed that ten progenies of the C. glabrata CBS138/ATCC2001 reference strain had a divergent expression of EPA family genes, resulting in altered adhesion properties. The low adherence strain CBS138/6 showed significant lower expression of EPA3, EPA6, EPA7 and EPA22 when compared to the other progenies (Bader, O., Schwarz, A. et al., 2012) indicating that the regulation of adhesins in C. glabrata is very dynamic. This adaptability may enable individual strains to adjust to different host cell conditions (Kraneveld, E. A., de Soet, J. J. et al., 2011).

The N-terminal part of the Epa proteins contain the binding domains that are similar in sequence to the so-called PA14 domains (anthrax protective antigen). Studies with glycan arrays indicated that PA14 of Epa1, Epa6 and Epa7 bind to oligosaccharides with terminal galactose residues, as they are found in mucin-type $O$-glycans (Zupancic, M. L., Frieman, M. et al., 2008). This finding supports the idea that Epa proteins can directly bind to glycoproteins on the surface of host cell (de Groot, P. W., Bader, O. et al., 2013).

Another important adhesin family in C. glabrata is the Pwp family. It consists of seven members that are lectins (de Groot, P. W., Bader, O. et al., 2013). In vitro studies showed that Pwp7 plays a role in adherence to human endothelial cells (Desai, C., Mavrianos, J. et al., 2011). Additionlly, six further non-Epa adhesin-like wall proteins were found in the C. glabrata cell wall, namely Awp1 to Awp6 (de Groot, P. W., Kraneveld, E. A. et al., 2008; Kraneveld, E. A., de Soet, J. J. et al., 2011). Awp1 is upregulated during biofilm development, and Awp6 was identified by mass spectrometric analysis in cell wall preparations of biofilms. It is assumed that expression of these adhesins depends on many factors which seem to be dependent on the genetic strain background, environmental growth conditions and aggregation (Kraneveld, E. A., de Soet, J. J. et al., 2011; de Groot, P. W., Bader, O. et al., 2013). However, the exact function and protein structure of the Pwp and Awp protein families still needs to be investigated. 


\subsubsection{Ecm33 protein family}

The Ecm33 protein family in C. glabrata consists of at least four members as Ecm33 (CAGL0M01826g), Pst1 (CAGL0E04620g), Sps2 (CAGL0H01661g), and Sps22 (CAGL0B00616g). Ecm 33 and Pst1 were found to be very abundant proteins in the cell wall of C. glabrata (de Groot, P. W., Kraneveld, E. A. et al., 2008). However, the exact function of the Ecm33 protein family is still unclear. Previous scientific evidences revealed that this family may play a role in cell wall remodeling, cell wall maintenance and cell biogenesis (de Groot, P. W., Ruiz, C. et al., 2001) in the ascomycocetes including S. cerevisiae (Pardo, M., Monteoliva, L. et al., 2004), C. albicans (Martinez-Lopez, R., Monteoliva, L. et al., 2004; Martinez-Lopez, R., Park, H. et al., 2006) and A. fumigatus (Chabane, S., Sarfati, J. et al., 2006).

In $S$. cerevisiae, deletion of ECM33 (YBR078w) resulted in hypersensitivity to cell wall perturbing agents and an increased amount of 1,6- $\beta$-glucan-linked proteins secreted to the culture medium. The deletion of PST1 (YDR055w), which is a homologue of ECM33, did not show these effects. However, synergistic effects affecting the cell wall were found in the double gene deletion mutant of ECM33 and PST1. The single gene deletion in ECM33 and the double gene deletion in ECM33 and PST1 resulted in a stimulation of phosphorylation in S1t2 protein which is a signaling controller of cell wall integrity (Pardo, M., Monteoliva, L. et al., 2004).

In C. albicans, the deletion mutant of ECM33 (orf19.3010.1) was sensitive to cell wall perturbing agents. It failed to form filaments in Spider medium and its blastospores were larger and more flocculated when compared to the wild-type strain. The cell wall architecture of the Aecm 33 mutant was abnormal because of the thickness of the internal layer of 1,3- $\beta$-glucan and chitin. ECM33 deletion reduced the adherence and invasion capacity to endothelial cells and the FaDu oral epithelial cell line. Moreover, the $\Delta$ ecm 33 mutant showed less virulence in a murine model indicating that the $C a E c m 33$ protein plays a role in $C$. albicans virulence (MartinezLopez, R., Monteoliva, L. et al., 2004; Martinez-Lopez, R., Park, H. et al., 2006; MartinezLopez, R., Nombela, C. et al., 2008; Rouabhia, M., Semlali, A. et al., 2012).

In the filamentous fungus A. fumigatus, the deletion of ECM33 results in altered phenotypes as compared to the wild-type strain: (i) rapid conidial germination (ii) a defect in conidial separation, (iii) an increase of conidia size with an increase in the amount of chitin in the cell wall, (iv) conidia were sensitive to the absence of aeration during long-term storage, (v) 
resistance to the cell pertubing agents, (vi) conidia were more resistant to killing by phagocytes, whereas the mycelium was more easily killed by neutrophils, and (vii) increased virulence in a mouse model. These results suggest that the $A f E c m 33$ protein contributes in key aspects of cell wall morphogenesis, cell wall integrity and plays a role in A. fumigatus virulence (Chabane, S., Sarfati, J. et al., 2006) (Romano, J., Nimrod, G. et al., 2006).

These primliminary findings indicate that the functionanl role of Ecm33 in S. cerivisiae is different from the function in A. fumigatus. However, so far there is no study that determinated the function of the abundant Ecm33 cell wall protein in the human pathogen C. glabrata.

Table 4. The effects of ECM33 deletion in $S$. cerevisiae, $C$. albicans, and A. fumigatus

\begin{tabular}{|c|c|c|c|}
\hline & \multicolumn{3}{|c|}{ Deletion of $E C M 33(\triangle e c m 33)^{*}$} \\
\hline & S. cerevisiae ${ }^{1}$ & C. albicans ${ }^{2-4}$ & A. fumigatus ${ }^{5-6}$ \\
\hline 1. Shape and size & rounder and larger & rounder and larger & larger \\
\hline 2. Cell perturbing agents & sensitive & sensitive & resistant \\
\hline 3. Cell wall architecture & $\begin{array}{l}\text { increased } 1,3 \text { and } 1,6-\beta- \\
\text { glucan levels }\end{array}$ & $\begin{array}{l}\text { increased } 1,3-\beta \text {-glucans } \\
\text { and chitin levels }\end{array}$ & increased chitin level \\
\hline 4. Hypha production & $\mathrm{N} / \mathrm{A}$ & absent $^{\mathrm{a}}$ & faster \\
\hline 5. Growth rate & ND & 2-fold reduced & increased \\
\hline 6. Flocculation & ND & yes & yes \\
\hline 7. Adherence in cell lines & ND & decreased & no significant difference \\
\hline 8. Pathogenicity in mouse & $\mathrm{N} / \mathrm{A}$ & decreased & increased \\
\hline
\end{tabular}

*: parental strain in each organism was used to compare a difference of phenotype.

1: (Pardo, M., Monteoliva, L. et al., 2004), ${ }^{2-4}$ : (Martinez-Lopez, R., Monteoliva, L. et al., 2004; Martinez-Lopez, R., Park, H. et al., 2006; Martinez-Lopez, R., Nombela, C. et al., 2008), and ${ }^{5-6}$ : (Chabane, S., Sarfati, J. et al., 2006; Romano, J., Nimrod, G. et al., 2006)

N/A: not applicable, ND: not determined.

a: yeast cells were grown in Spider medium for 7 days in $37^{\circ} \mathrm{C}$. 


\subsection{Aims of the study}

C. glabrata is the second most frequently observed yeast in clinical specimen. In comparison to C. albicans infections, candidiasis caused by C. glabrata is more difficult to treat because this species is often resistant to azole antifungals and displays a high degree of adhesive capacities on clinically used catheter materials, facilitating the formation of biofilms.

Proteins of the Ecm33/Pst1 family in C. glabrata were experimentally shown to be highly abundant cell wall proteins of this organism. Previous studies indicate that the ECM33 homologs in S. cerevisiae, C. albicans and A. fumigates are associated with integrity and architecture of the cell wall, adherence capacity, growth rate and with virulence, but the true molecular function of the proteins in these organisms is unknown. Furthermore, phenotypic analysis of ECM33 deletion mutants in the different species indicates that the functional role of Ecm33 homologs might be different between the organisms. In C. glabrata the cell wall is of particular interest, because it holds key functions such as adhesion and detoxification of immune responses (e.g. the oxidative burst of attacking macrophages) during pathogenesis. Therefore, this study focuses on the characterization of the Ecm33 protein family of C. glabrata, where the cell wall architecture is significantly different to otherwise closely related fungi. In C. glabrata, this family consists of four members: Ecm33, Pst1, Sps2, and Sps22.

The aims of the present study were (i) to generate molecular tools for the study of this gene family in $C$. glabrata such as mutants, revertants, complemented strains, recombinant Ecm33-family proteins, and anti-Ecm33 antibodies, (ii) to characterize the function of Ecm33 and Pst1 of C. glabrata in relation to their orthologs in C. albicans and $S$. cerevisiae via comparison of the phenotypic differences among the ECM33 and PST1 deleted mutants and wild-type in all three organisms, and (iii) to gather data on the biological function of Ecm33 protein in C. glabrata by using the generated molecular tools. 


\section{CHAPTER 2}

\section{MATERIALS AND METHODS}

\subsection{Disposables}

Disposables were purchased from Sarstedt (Nümbrecht, Germany), Greiner bio-one (Frickenhausen, Germany), Corning (Berlin, Germany), and Eppendorf (Hamburg, Germany).

\subsection{Chemicals}

- General chemicals were purchased from Roth (Karlsruhe, Germany), Merck (Darmstadt, Germany), and Sigma-Aldrich (Munich, Germany).

- Restriction enzymes were purchased from New England BioLabs Inc (Frankfurt am Main, Germany).

- PCR purification and agarose gel extraction kits were purchased from Qiagen (Hilden, Germany)

- DNA and protein weight standards were purchased from Fermentas (Vilnius, Lithuania)

- Oligonucleotides were purchased from Sigma-Aldrich (Munich, Germany).

- Reagents for Real-time PCR (LightCycler ${ }^{\circledR}$ ) were purchased from Roche (Mannheim, Germany)

\subsection{Media}

All liquid media were sterilized at $121^{\circ} \mathrm{C}$ for 15 minutes or by filtration $(0.22 \mu \mathrm{m}$ pore size) and were kept at $4^{\circ} \mathrm{C}$.
LB $1 \%[\mathrm{w} / \mathrm{v}]$ Bacto-tryptone, $0.5 \%[\mathrm{w} / \mathrm{v}]$ yeast extract, $0.5 \%[\mathrm{w} / \mathrm{v}] \mathrm{NaCl}, 2 \%[\mathrm{w} / \mathrm{v}]$ agar (for $\mathrm{LB}$ agar) in $\mathrm{ddH}_{2} \mathrm{O}$.


For selection LB media, ampicillin $(100 \mu \mathrm{g} / \mathrm{ml})$ or Zeocin ${ }^{\mathrm{TM}}(25 \mu \mathrm{g} / \mathrm{ml})$ was added into media after autoclaving.

YPD $1 \%[\mathrm{w} / \mathrm{v}]$ yeast extract, $2 \%[\mathrm{w} / \mathrm{v}]$ peptone, $2 \%[\mathrm{w} / \mathrm{v}]$ dextrose, $2 \%[\mathrm{w} / \mathrm{v}]$ agar (for YPD agar) in $\mathrm{ddH}_{2} \mathrm{O}$.

For selection YPD media, Zeocin ${ }^{\mathrm{TM}}(200 \mu \mathrm{g} / \mathrm{ml})$ was added into media after autoclaving.

MM $1.34 \%[\mathrm{w} / \mathrm{v}] \mathrm{YNB}, 2 \%[\mathrm{w} / \mathrm{v}]$ dextrose, $0.006 \%[\mathrm{w} / \mathrm{v}]$ histidine, $0.008 \%$ [w/v] leucine, $0.026 \%[\mathrm{w} / \mathrm{v}]$ tryptophan, $2 \%[\mathrm{w} / \mathrm{v}]$ agar (for MM agar) in $\mathrm{ddH}_{2} \mathrm{O}$.

RPMI $16401.04 \%$ [w/v] RPMI-1640, 2\% [w/v] dextrose, 3.453\% [w/v] 3-(N-morpholino)propanesulphonic acid (MOPS) in $\mathrm{ddH}_{2} \mathrm{O}$.

BMMH $100 \mathrm{mM}$ potassium phosphate $\mathrm{pH} 6,1.34 \%$ [w/v] YNB with ammonium sulfate, $0.00004 \%[\mathrm{w} / \mathrm{v}]$ biotin, $0.5 \%[\mathrm{v} / \mathrm{v}]$ methanol, $0.004 \%[\mathrm{w} / \mathrm{v}]$ histidine in $\mathrm{ddH}_{2} \mathrm{O}$.

\subsection{Plasmids}

Table 5. Plasmids used in this study

\begin{tabular}{lll}
\hline Code & Plasmid & Source \\
\hline P1 & pQE30 & Qiagen \\
P2 & pQE30:CgECM33 & This study \\
P3 & pPICZ $\alpha$ & Invitrogen \\
P4 & pPICZ $\alpha: C g E C M 33$ & This study \\
P5 & pPICZ $\alpha: C g P S T 1$ & This study \\
P6 & pBM51-Leu & Schwarzmüller T., Vienna* \\
P7 & pBM51-Leu:CgECM33 & This study \\
P8 & pBM51-Leu:CgPST1 & This study \\
P9 & pBM51-Leu:CgSPS2 & This study \\
P10 & pBM51-Leu:CgSPS22 & This study \\
P11 & pBM51-Leu:truncated CgECM33 & This study \\
P12 & pBM51-Leu:ScECM33 & This study \\
P13 & pBM51-Leu:CaECM33 & This study \\
\hline
\end{tabular}

*: (Schwarzmüller, T., 2009) 


\section{$2.5 E$. coli strains}

Table 6. E. coli strains used in this study

\begin{tabular}{|c|c|c|c|c|}
\hline Code & Strains & Plasmid & Gene of interest & Description \\
\hline 1 & DH5 $\alpha$ & - & - & Empty host \\
\hline 1.1 & BL-21 & - & - & Empty host \\
\hline 1.2 & Rosetta & - & - & Empty host \\
\hline 2 & $\mathrm{DH} 5 \alpha$ & pQE30 & - & Amplification of pQE30 \\
\hline 3 & $\mathrm{DH} 5 \alpha$ & $\mathrm{pPICZ} \alpha$ & - & Amplification of pPICZ $\alpha$ \\
\hline 4 & $\mathrm{DH} 5 \alpha$ & $\mathrm{pPICZ} \alpha$ & CgECM33 & Amplification of pPICZ $\alpha: C g E C M 33$ \\
\hline 5 & $\mathrm{DH} 5 \alpha$ & $\mathrm{pPICZ} \alpha$ & CgPST1 & Amplification of pPICZ $\alpha: C g P S T 1$ \\
\hline 6 & $\mathrm{DH} 5 \alpha$ & pQE30 & CgECM33 & Amplification of pQE30:CgECM33 \\
\hline 7 & BL-21 & pQE30 & CgECM33 & Expression of Ecm $33 p$ in E. coli \\
\hline 8 & Rosetta & pQE30 & CgECM33 & Expression of Ecm $33 p$ in E. coli \\
\hline 11 & DH5a & pBM51-Leu & - & Amplification of pBM51-Leu \\
\hline 15 & DH5a & pBM51-Leu & ECM33 & Amplification of pBM51-Leu:CgECM33 for revertant construction \\
\hline 17 & DH5a & pBM51-Leu & PST1 & Amplification of pBM51-Leu:CgPST1 for revertant construction \\
\hline 25 & $\mathrm{DH} 5 \alpha$ & pBM51-Leu & ScECM33 & Amplification of pBM51-Leu:ScECM33 for complemented strain construction \\
\hline 26 & $\mathrm{DH} 5 \alpha$ & pBM51-Leu & CaECM33 & Amplification of pBM51-Leu:CaECM33 for complemented strain construction \\
\hline 27 & $\mathrm{DH} 5 \alpha$ & pBM51-Leu & truncated $C g E C M 33$ & Amplification of pBM51-Leu:truncated $C g E C M 33$ for complemented strain construction \\
\hline 28 & $\mathrm{DH} 5 \alpha$ & pBM51-Leu & $\operatorname{CgSPS} 2$ & Amplification of pBM51-Leu:CgSPS2 for complemented strain construction \\
\hline 29 & DH5 $\alpha$ & pBM51-Leu & $\operatorname{CgSPS} 22$ & Amplification of pBM51-Leu:CgSPS22 for complemented strain construction \\
\hline
\end{tabular}




\subsection{Yeast strains}

Table 7. Yeast strains used in this study

\begin{tabular}{|c|c|c|c|c|}
\hline Code & Organisms & Strains & Source and reference & Description \\
\hline Y15 & P. pastoris & $\mathrm{X} 33$ & Invitrogen & Wild-type \\
\hline Y16 & P. pastoris & GS115 & Invitrogen & his4 \\
\hline Y17 & P. pastoris & KM71H & Invitrogen & $\arg 4 a o x 1: A R G 4$ \\
\hline Y18 & P. pastoris & GS115:Albumin & Invitrogen & Control for secreated protein \\
\hline Y19 & P. pastoris & GS115:LacZ & Invitrogen & Control for intracellular protein \\
\hline Y20 & P. pastoris & GS115: 1,6- $\beta$-glucanase & Bom IJ., Vlaardingen ${ }^{1}$ & $1,6-\beta$-glucanase \\
\hline Y21 & P. pastoris & GS115:CgECM33 & This study & Ecm 33 protein expression \\
\hline Y22 & P. pastoris & GS115:CgPST1 & This study & Pst1 protein expression \\
\hline Y23 & P. pastoris & $\mathrm{X} 33: C g E C M 33$ & This study & Ecm33 protein expression \\
\hline Y24 & P. pastoris & $\mathrm{X} 33: C g P S T 1$ & This study & Pst1 protein expression \\
\hline Y25 & S. cerevisiae & Auxotroph BY4741 & Gil C., Madrid ${ }^{2}$ & Background wild-type \\
\hline Y26 & S. cerevisiae & $\Delta e c m 33$ & Gil C., Madrid ${ }^{2}$ & FBEHO41-01A \\
\hline Y27 & S. cerevisiae & $\Delta e c m 33$ & Gil C., Madrid ${ }^{2}$ & BY4741 \\
\hline Y28 & S. cerevisiae & $\Delta p s t 1$ & Gil C., Madrid ${ }^{2}$ & BY4741 \\
\hline Y29 & S. cerevisiae & 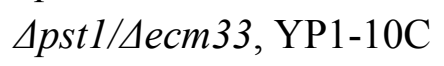 & Gil C., Madrid ${ }^{2}$ & FBEHO41-01A \\
\hline Y30 & S. cerevisiae & 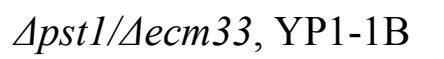 & Gil C., Madrid ${ }^{2}$ & FBEHO41-01A \\
\hline Y31 & C. albicans & Clinical strain & NRZ, Göttingen & Pathogen \\
\hline Y34 & C. glabrata & CBS138/ATCC2001 & Schwarzmüller T., Vienna ${ }^{3}$ & Wild-type \\
\hline Y35 & C. glabrata & Auxotroph $\Delta h t l$ & Schwarzmüller T., Vienna ${ }^{3}$ & Background wild-type \\
\hline Y36 & C. glabrata & $\Delta p s t 1$ & Schwarzmüller T., Vienna & Single deletion mutant \\
\hline Y37 & C. glabrata & $\Delta e c m 33$ & Schwarzmüller T., Vienna & Single deletion mutant \\
\hline Y40 & C. glabrata & $\Delta p s t 1 / \Delta e c m 33$ & Schwarzmüller T., Vienna & Double deletion mutant \\
\hline
\end{tabular}


Table 7. Yeast strains used in this study (continued)

\begin{tabular}{|c|c|c|c|c|}
\hline Code & Organisms & Strains & Source or reference & Description \\
\hline Y43 & C. glabrata & $\triangle p s t 1: C g P S T 1$ & This study & Completed revertant \\
\hline Y45 & C. glabrata & $\triangle e c m 33: C g E C M 33$ & This study & Completed revertant \\
\hline Y47 & C. glabrata & $\Delta p s t 1 / \Delta e c m 33: C g P S T 1$ & This study & Incompleted revertant \\
\hline Y49 & C. glabrata & 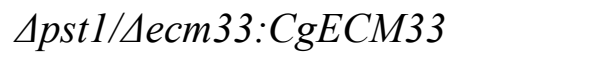 & This study & Incompleted revertant \\
\hline Y51 & C. albicans & Auxotroph Caf2 & Gil C., Madrid ${ }^{4}$ & Background wild-type \\
\hline Y53 & C. albicans & 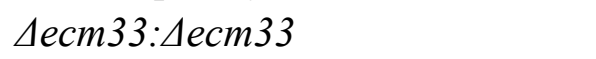 & Gil C., Madrid ${ }^{4}$ & Homozygous mutant \\
\hline Y55 & C. albicans & 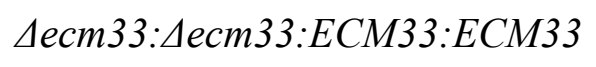 & Gil C., Madrid $^{4}$ & Revertant \\
\hline Y60 & C. glabrata & $\triangle e c m 33: S c E C M 33$ & This study & Complemented strain \\
\hline Y62 & C. glabrata & $\triangle e c m 33: C a E C M 33$ & This study & Complemented strain \\
\hline Y64 & C. glabrata & $\triangle e c m 33: C g P S T 1$ & This study & Complemented strain \\
\hline Y65 & C. glabrata & $\triangle e c m 33:$ truncated $C g E C M 33$ & This study & Complemented strain \\
\hline Y67 & C. glabrata & $\triangle e c m 33: C g S P S 2$ & This study & Complemented strain \\
\hline Y68 & C. glabrata & $\triangle e c m 33: C g S P S 22$ & This study & Complemented strain \\
\hline
\end{tabular}

${ }^{1}$ : (Bom, I. J., Dielbandhoesing, S. K. et al., 1998)

: (Pardo, M., Monteoliva, L. et al., 2004)

: (Schwarzmüller, T., 2009)

4: (Martinez-Lopez, R., Monteoliva, L. et al., 2004).

NRZ: National Reference Center for Systemic Mycoses, Göttingen, Germany 


\subsection{Oligonucleotides}

\section{Table 8. Oligonucleotides used in this study}

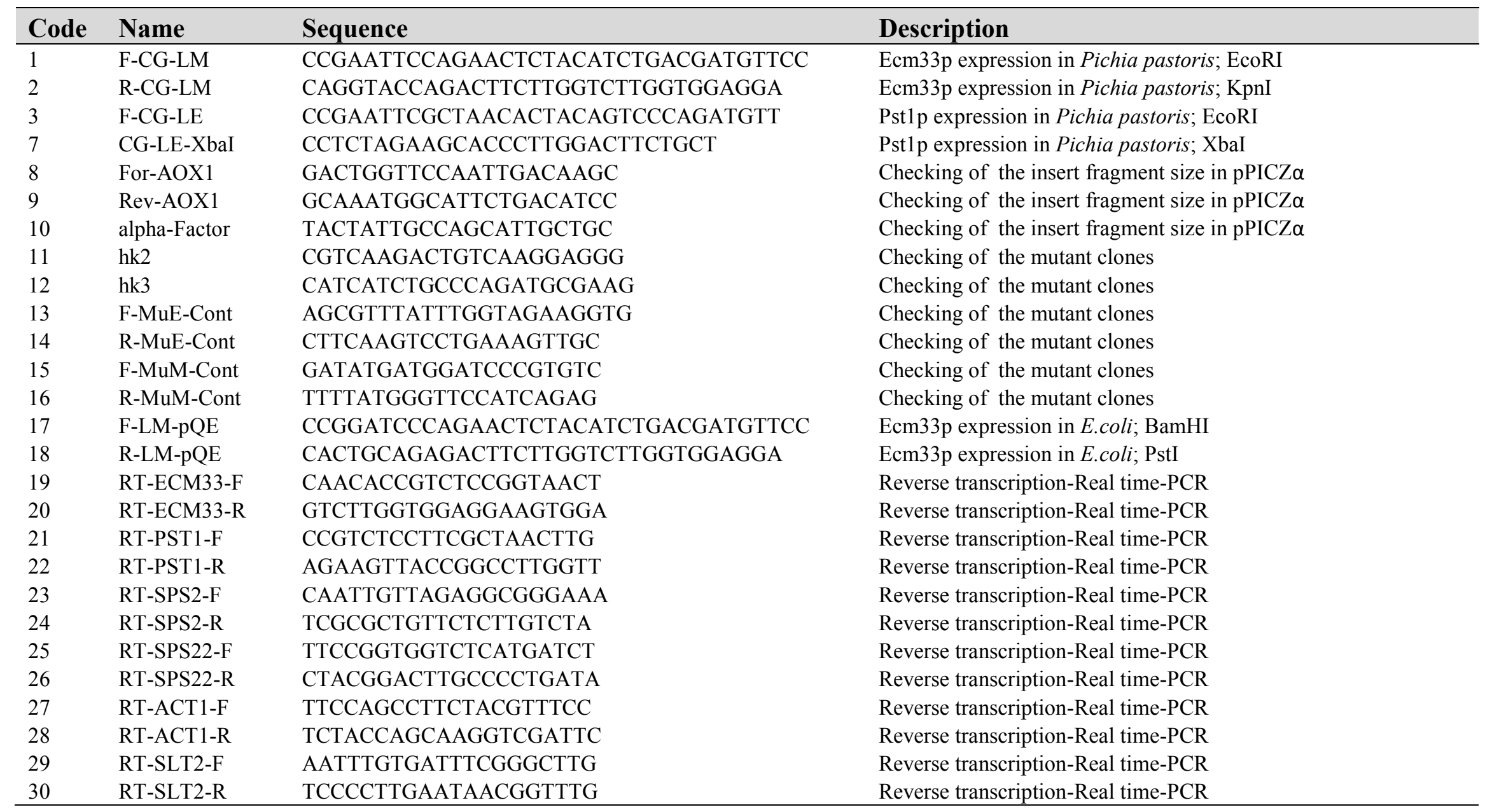


Table 8. Oligonucleotides used in this study (continued)

\begin{tabular}{|c|c|c|c|}
\hline Code & Name & Sequence & Description \\
\hline 31 & 1F-Rev-ECM & CCCTCGAGATGAAGGTCACATCATTGGT & Generation of $E C M 33$ revertant; XhoI \\
\hline 32 & 2R-Rev-ECM & CAGGATCCTCATAGCAAAGCGACGGCGA & Generation of ECM33 revertant; BamHI \\
\hline 33 & 3F-Rev-PST & CCCTCGAGATGCAATTGAATCATCTTTTG & Generation of PST1 revertant; XhoI \\
\hline 34 & 4R-Rev-PST & CAGGATCCCTATATTAGAGCCAAAGCAAC & Generation of $P S T 1$ revertant; BamHI \\
\hline 43 & M13 F & GTAAAACGACGGCCAGT & For Topo2.1 sequencing \\
\hline 44 & M13 R & CAGGAAACAGCTATGAC & For Topo 2.1 sequencing \\
\hline 53 & F-Seq & GTTTTCCCAGTCACGACGTT & Checking of the inserted fragment in pBM51-Leu \\
\hline 54 & R-Seq & CCAAGGGGGTGGTTTAGTTT & Checking of the inserted fragment in pBM51-Leu \\
\hline 57 & $\mathrm{~F} \mathrm{Ca} \mathrm{Xho}$ & CACTCGAGATGCAATTCAAGAACATTCTTGC & Generation of $C a E C M 33$ complemented strain; XhoI \\
\hline 58 & R_Ca_Bam & CAGGATCCTTAGAATAAAGCAACACCAACAGC & Generation of CaECM33 complemented strain; BamHI \\
\hline 61 & F Sc Xho & CACTCGAGATGCAATTCAAGAACGCTTTGAC & Generation of ScECM33 complemented strain; XhoI \\
\hline 62 & R_Sc_Bam & CAGGATCCTTATAGTAAGGCAACGCCAACAG & Generation of ScECM33 complemented strain; BamHI \\
\hline 69.2 & F-CgSps22_Sal & CCGTCGACATGAAGCTTATTTTTGTTGC & Generation of CgSPS22 complemented strain; SalI \\
\hline 70 & R-CgSps 22 Bam & CCGGATCCCTAAAAAAATAGTGCACTCG & Generation of CgSPS22 complemented strain; BamHI \\
\hline 71.2 & F-CgSps2_Xho & CACTCGAGATGCACTGCAAGTCACCAAAC & Generation of $C g S P S 2$ complemented strain; XhoI \\
\hline 72 & R-CgSps $2-$ Bam & CCGGATCCTTAAAACAGTATCAAATACATG & Generation of $C g S P S 2$ complemented strain; BamHI \\
\hline 75 & R_truncatedECM & CAGGATCCAGACTTCTTGGTCTTGGTGGAGGA & Generation of truncated $C g E C M 33$ complemented strain; BamHI \\
\hline 76 & RT-R-Ca & GAACGGTGCCACATCTACCT & Reverse transcription-Real time-PCR \\
\hline 77 & RT-F-Ca & CTGGAGCAGCACCCTTAGAC & Reverse transcription-Real time-PCR \\
\hline 78 & RT-R-Sc & TGCTAACGGTTCATTTGCTG & Reverse transcription-Real time-PCR \\
\hline 79 & RT-F-Sc & ATGTTGACGGAACCACCAAT & Reverse transcription-Real time-PCR \\
\hline
\end{tabular}




\subsection{In silico analysis}

Ecm33, Pst1, Sps2, and Sps22 protein sequences of C. glabrata were taken from Genomic Exploration of the Hemiascomycete Yeasts (http://www.genolevures.org) and aligned with MEGA4 program. Signal peptide were predicted by using SignalP (http://www.cbs.dtu.dk/services/SignalP/). Omega sites for GPI anchor attachment were predicted by using the big-PI Predictor server (http://mendel.imp.ac.at/gpi/gpi_server.html).

The following programs were used in this study:

- Saccharomyces Genome Database (SGD) (http://www.yeastgenome.org)

- Candida Genome Database (CGD) (http://www.candidagenome.org)

- Genomic Exploration of the Hemiascomycete Yeasts (http://www.genolevures.org)

- Basic Local Alignment Search Tool (BLAST) (http://blast.ncbi.nlm.nih.gov)

- The translation of a nucleotide (DNA/RNA) sequence to a protein sequence (http://web.expasy.org/translate/)

- Protein Molecular Weight Calculator (http://www.sciencegateway.org/tools/proteinmw.htm)

- NEBcutter V2.0 (http://tools.neb.com/NEBcutter2/)

- Oligo Calc: Oligonucleotide Properties Calculator (http://www.basic.northwestern.edu/biotools/oligocalc.html)

- Primer 3 program for designing PCR primers (http://frodo.wi.mit.edu)

- PlasMapper Version 2.0 for plasmid drawing (http://wishart.biology.ualberta.ca/PlasMapper/index.html)

- SignalP 4.1 server for signal peptide prediction (http://www.cbs.dtu.dk/services/SignalP/)

- big-PI Predictor for GPI Modification Site Prediction (http://mendel.imp.ac.at/gpi/gpi_server.html) 


\subsection{Generation of knock out strains}

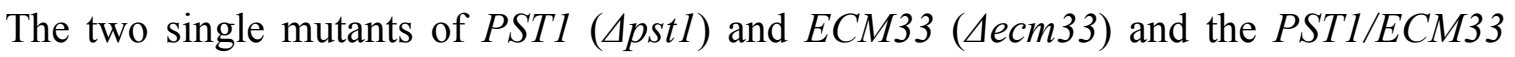
double mutant (Apst1/4ecm33) in C. glabrata were generated and provided by Tobias Schwarzmüller, Vienna, Austria.

Briefly, fusion PCR was used to generate both single mutants and double mutant. The dominant marker NAT1 was amplified from plasmid pJK863 (Shen, J., Guo, W. et al., 2005) with primers fp_NAT1-U2 (5'-CGTACGCTGCAGGTCGACagcttgcctcgtccecgccg-3') and rp_NAT1D2 (5'-CTACGAGACCGACACCGctggatggeggegttagtatcg-3') to add the two 20 bp adaptor sequences U2 and D2. The PCR product was ligated into pGEM-T vector (Promega, Mannheim, Germany), generating plasmid pTS50.

Fusion PCR was used to generate the deletion cassettes (Wach, A., 1996; Noble, S. M. and Johnson, A. D., 2005) (Figure 5 A). The 500 bp long flanking homology regions were amplified from C. glabrata strain ATCC2001 genomic DNA with primer pairs 5'5'/5'3' and 3'5'/3'3' adding the adaptor sequence (U1/D1) of $20 \mathrm{bp}$ and purified by ethanol precipitation. A $50 \mu$ reaction $\left(50 \mathrm{mM} \mathrm{KCl}, 10 \mathrm{mM}\right.$ Tris- $\mathrm{HCl}\left(\mathrm{pH} 9.0,25^{\circ} \mathrm{C}\right), 0.1 \%$ TritionX-100, $1.5 \mathrm{mM}$ $\mathrm{MgCl} 2,0.2 \mu \mathrm{M}$ dNTPs, $0.5 \mu \mathrm{M}$ each primer, 1 unit Taq-Polymerase and genomic wild-type DNA from strain ATCC2001) was subjected to cycling $\left(93^{\circ} \mathrm{C}\right.$ for 5 minutes, 35 cycles $93^{\circ} \mathrm{C}$ for 30 seconds, $45^{\circ} \mathrm{C}$ for 30 seconds, $72^{\circ} \mathrm{C}$ for 90 seconds, finally 10 minutes at $72^{\circ} \mathrm{C}$ ) in a thermocycler machine.

The dominant marker NAT1 was amplified from plasmid pTS50 in a separated PCR reaction using primers $5 \mathrm{M}$ and $3 \mathrm{M}$, adding unique barcode tags and complementary $\mathrm{U} 1$ and D1 adaptor sequences (Figure $5 \mathrm{~B}$ ). The marker fragment was gel-purified over a $0.7 \%$ agarose gel.

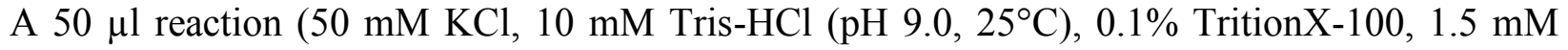
MgCl2, $0.2 \mu \mathrm{M}$ dNTPs, $0.5 \mu \mathrm{M}$ each primer, 1 unit Taq-Polymerase and plasmid TS50) was subjected to cycling $\left(93^{\circ} \mathrm{C}\right.$ for 3 minutes, 32 cycles $93^{\circ} \mathrm{C}$ for 30 seconds, $49^{\circ} \mathrm{C}$ for 30 seconds, $72^{\circ} \mathrm{C}$ for 2.5 minutes, finally 10 minutes at $72^{\circ} \mathrm{C}$ ) in a thermocycler machine.

The fusion PCR was carried out in a $50 \mu \mathrm{l}$ volume with the same condition as above (1x

ExTaq buffer, $0.2 \mu \mathrm{M}$ dNTPs, $0.5 \mu \mathrm{M}$ each primer, 2.5 units ExTaq-Polymerase (TaKaRa, Darmstadt, Germany) and $3 \mu \mathrm{l}$ marker fragment, and $1.25 \mu \mathrm{l}$ each flanking homology fragment) and subjected to cycling $\left(93^{\circ} \mathrm{C}\right.$ for 3 minutes, 35 cycles $93^{\circ} \mathrm{C}$ for 30 seconds, $45^{\circ} \mathrm{C}$ for 30 
seconds, $72^{\circ} \mathrm{C}$ for 3 minutes, finally 10 minutes at $72^{\circ} \mathrm{C}$.) The final deletion construct was purified by ethanol precipitation (Schwarzmüller, T., 2009).

A

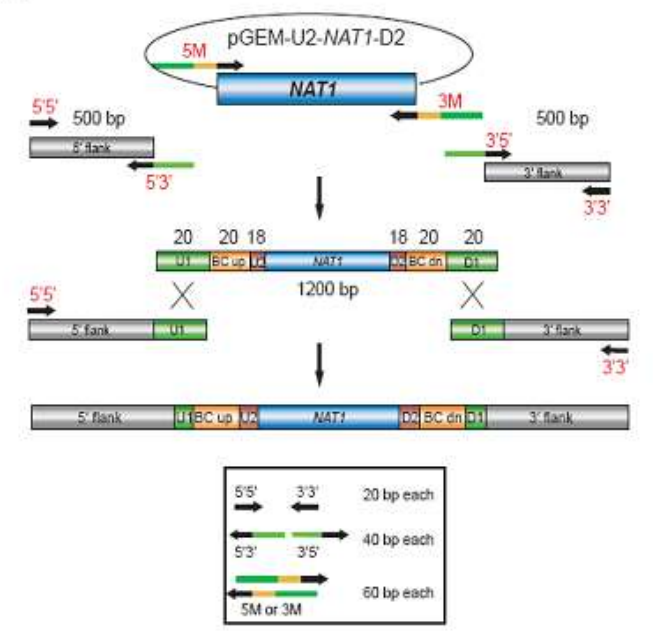

B

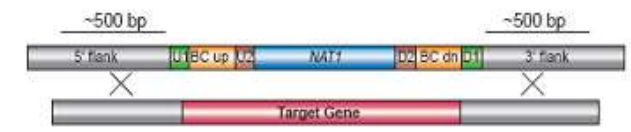

C
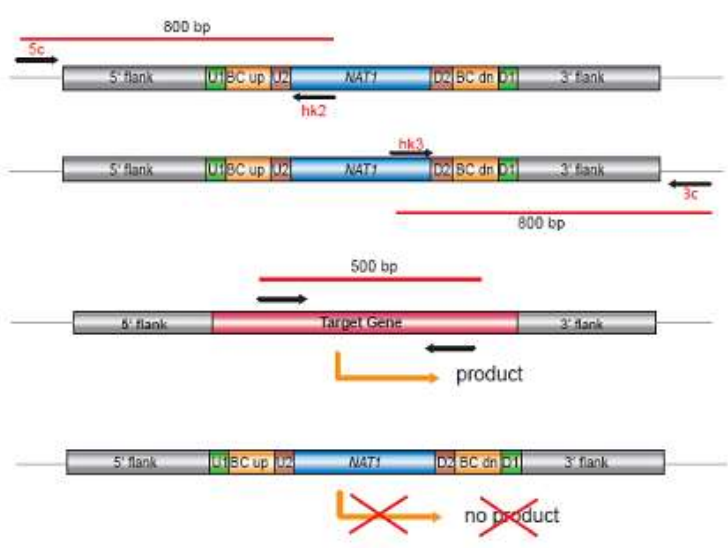

Figure 5. The sequential steps for generation of $C$. glabrata gene deletion cassettes

(A): Fusion PCR using the dominant selectable marker NAT1 was applied to generate gene deletion cassettes. The 5' and 3' flanking sequences of target genes were integrated in oligonucleotides to amplify the marker fragment and to add overlap sequences. (B): Gene disruption by replacement of the target gene based on homologous recombination. C. glabrata strain $\Delta h t l$ was transformed by an electroporation method. (C): Verification of correct integration of the deletion cassette by colony PCR (forward primer outside flanking region and reverse primer inside marker) checking both junctions. Loss-of-gene PCR (internal primer pair) was used to confirm that the coding sequences has not reintegrated in the genome. Reintegration, indicating that the CDS remained in the genome, gave a $500 \mathrm{bp}$ PCR product. Figure taken from (Schwarzmüller, T., 2009).

\subsection{Generation of revertants and complemented strains in $C$. glabrata}

The set of full revertants (Apst1:CgPST1 and $\triangle$ ecm33:CgECM33), incompleted revertants (Apst1/Aecm33:CgPST1 and $\triangle p s t 1 / \triangle e c m 33: C g E C M 33)$ and complemented strains

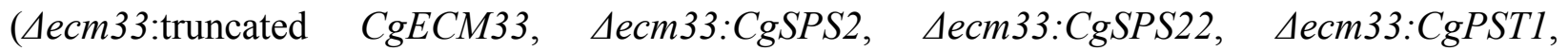
Aecm33:CaECM33 and Aecm33:ScECM33) were generated by using the target gene cloned to pBM51-Leu. The expression plasmid contains an autonomously replicating sequence (ARS), a 
centromere (CEN), a leucine selectable marker of C. glabrata and a PGK1 (3-phosphoglycerate kinase 1) promotor of $S$. cerevisiae ( $S c P G K 1$ ) as shown in Figure 6. This system can restore gene expression in the generated knock out strains (Ma, B., Pan, S. J. et al., 2007; Schwarzmüller, T., 2009).

All of the inserted fragments were amplified with specific primers including adaptors for restriction enzyme sites from CBS138/ATCC2001 genomic DNA. A $50 \mu 1$ reaction (5 $\mu 1$ of 10x KOD Taq buffer, $5 \mu 1$ of $2 \mathrm{mM}$ dNTPs, $3 \mu 1$ of $25 \mathrm{MgCl}_{2}, 1 \mu 1$ of $20 \mu \mathrm{M}$ each primer, $1 \mu 1$ of KOD Taq-Polymerase (1U/ $\mu 1$; Novagen, Darmstadt, Germany), and $2 \mu 1$ of genomic DNA (50 $\mathrm{ng} / \mu \mathrm{l})$ ) was subjected to cycling $\left(95^{\circ} \mathrm{C}\right.$ for 5 minutes, 35 cycles of $95^{\circ} \mathrm{C}$ for 30 seconds, $\mathrm{XX}^{\circ} \mathrm{C}$ (see annealing temperature in Table 9) for 30 seconds, $70^{\circ} \mathrm{C}$ for 30 seconds, finally 5 minutes at $70^{\circ} \mathrm{C}$ in a thermocycler machine. The size of the inserted PCR products are shown in Table 9.

The pBM51-Leu and the inserted fragments were digested with a restriction enzyme. After digestion, the fragments and linearized pBM51-Leu plasmid were purified by using QIAquick Gel Extraction Kit (Qiagen). The ligation between the fragments and linearized pBM51-Leu plasmid were linked by using T4 DNA ligase (Thermo Scientific, MA, USA) as follows: linear vector $50 \mathrm{ng}$, inserted DNA fragment $250 \mathrm{ng}, 2 \mu \mathrm{l}$ of 10X T4 DNA ligase buffer and $1 \mu 1$ of T4 DNA ligase $(1 \mathrm{U} / \mu 1)$. The mixtures were incubated at $25^{\circ} \mathrm{C}$ for 1 hour and kept in $4^{\circ} \mathrm{C}$ for overnight. Five $\mu 1$ of the mixture was used for transformation in the competent DH5 $\mathrm{E}$. coli using a heat shock transformation protocol (see the detail protocol in 2.10 .1 and 2.10.2). The transformant $\mathrm{DH} 5 \alpha$ E. coli was selected by using ampicillin drug resistance.

The positive DH5 $\alpha$ clones were verified with two approaches as restriction enzyme digestion and DNA sequencing.

Plasmids from pBM51-Leu no. P7-P13 were extracted and used to transform C. glabrata by electroporation (see the detail protocol in 2.10 .3 and 2.10.4). C. glabrata transformats were selected by growth on minimal medium agar without leucine. 


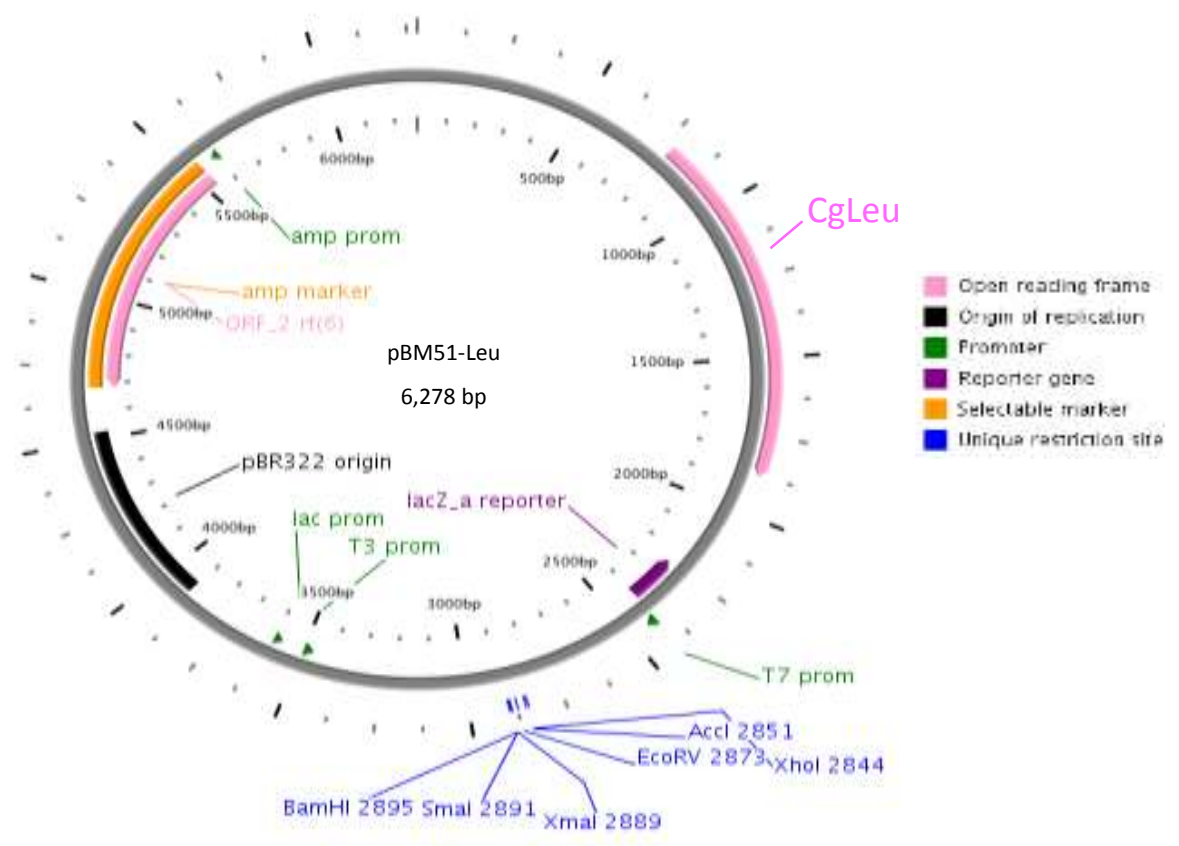

Figure 6. Map of pBM51-Leu

pBM51-Leu map was drawn from online program PlasMapper Version 2.0 (http://wishart.biology.ualberta.ca/PlasMapper/index.html). The pBM51-Leu can be replicated in E. coli using pBR322 origin of replication and expressed in S. cerevisiae and C. glabrata using strong promotor of $S$. cerevisiae. The pBM51-Leu's multiple cloning site contains the following restriction sites: XhoI, AccI, EcoRV, XmaI, SmaI, and BamHI. The marker for selection in E. coli is ampicillin resistant and in yeast cell is leucine production.

Table 9. Generation of revertants and complemented strains in C. glabrata

\begin{tabular}{ccccc}
\hline Gene & $\begin{array}{c}\text { Size } \\
(\mathrm{bp})\end{array}$ & Primer code & $\begin{array}{c}\text { Annealing temp. } \\
\left({ }^{\circ} \mathrm{C}\right)\end{array}$ & $\begin{array}{c}\text { Restriction } \\
\text { enzyme }\end{array}$ \\
\hline$C g E C M 33$ & 1266 & 31 and 32 & 55 & Xhol/BamHI \\
CgPST1 & 1290 & 33 and 34 & 55 & Xhol/BamHI \\
truncated $C g E C M 33$ & 1185 & 31 and 75 & 55 & Xhol/BamHI \\
$C g S P S 2$ & 1251 & 71.2 and 72 & 58 & Xhol/BamHI \\
$C g S P S 22$ & 1377 & 69.2 and 70 & 58 & Sall/BamHI \\
CaECM33 & 1272 & 57 and 58 & 60 & Xhol/BamHI \\
ScECM33 & 1290 & 61 and 62 & 60 & Xhol/BamHI \\
\hline
\end{tabular}




\subsubsection{Preparation of competent $E$. coli}

A single colony of $E$. coli was grown in $5 \mathrm{ml} \mathrm{LB}$ in glass tube at $37^{\circ} \mathrm{C}$ for overnight with shaking. One $\mathrm{ml}$ of overnight culture was inoculated into $100 \mathrm{ml} \mathrm{LB}$ in a $250 \mathrm{ml}$ flask and incubated at $37^{\circ} \mathrm{C}$ for $1.5-3$ hours until an OD600 of 0.5 . These cells were put on ice for 15 minutes and the cells were collected by centrifugation for 3 minutes at 1,942 x g (3,000 rpm). The cell pellet was treated with cold $0.1 \mathrm{M} \mathrm{CaCl}_{2}$, incubated on ice for 20 minutes and centrifuged to keep the cell pellet. The cell pellet was resuspended with $2 \mathrm{ml}$ cold $0.1 \mathrm{M} \mathrm{CaCl}_{2}$ in $20 \%$ glycerol and aliquots of $100 \mu \mathrm{l}$ competent cells were stored at $-80^{\circ} \mathrm{C}$ (Sambrook, J., Russell, D.W., 2001).

\subsubsection{Transformation of $E$. coli}

Fifty ng of circular plasmid was added into $100 \mu \mathrm{l}$ of competent E.coli suspension and incubated on ice for 30 minutes. Then a heat shock was applied to the cells for 2 minutes at $42^{\circ} \mathrm{C}$ in a water bath before the cells were put back on ice. $500 \mathrm{ml}$ of LB was added to the tube and incubated at $37^{\circ} \mathrm{C}$ for an hour and then plated on LB agar with selective antibiotic drug (Sambrook, J., Russell, D.W., 2001).

\subsubsection{Preparation of competent yeast cell}

A combination of procedures modified from three protocols was used to prepare the competent C. glabrata and P. pastoris cells (Wu, S. and Letchworth, G. J., 2004; Lin-Cereghino, J., Wong, W. W. et al., 2005; Gietz, R. D. and Schiestl, R. H., 2007). A single colony of yeast was grown overnight in $5 \mathrm{ml} \mathrm{YPD}$ in a glass tube at $30^{\circ} \mathrm{C}$ with shaking. One $\mathrm{ml}$ of an overnight culture was inoculated into $100 \mathrm{ml}$ YPD in $250 \mathrm{ml}$ flask and incubated additionally at $30^{\circ} \mathrm{C}$ for 3-5 hours until an OD600 of 0.8 - 1.0 was reached. The cells were put on ice for 15 minutes and harvested by centrifugation for 3 minutes at $486 \times \mathrm{g}(1,500 \mathrm{rpm})$. The cell pellet was treated with $10 \mathrm{ml}$ cold transformation buffer $(7.9 \mathrm{ml}$ of TEDS, $2 \mathrm{ml}$ of $500 \mathrm{mM}$, and $100 \mu \mathrm{l}$ of $1 \mathrm{M}$ Dithiothreitol (DTT)), incubated on ice for 15 minutes and centrifuged to result in a cell pellet. 
The cell pellet was washed with $10 \mathrm{ml}$ cold $1 \mathrm{M}$ Sorbitol and resuspended with $2 \mathrm{ml}$ cold $1 \mathrm{M}$ Sorbitol. Aliquots of $100 \mu \mathrm{l}$ yeast competent cells were stored at $-80^{\circ} \mathrm{C}$

\subsubsection{Electroporation of yeast}

$5 \mu \mathrm{l}(50-100 \mathrm{ng})$ of PmeI linearized plasmid in case of P. pastoris or circular plasmid in case of C. glabrata and $100 \mu \mathrm{l}$ of competent yeast cells was mixed together and loaded into a 1 $\mathrm{mm}$ gap electroporation cuvette (PeqLab Biotechnologie $\mathrm{GmbH}$, Erlangen, Germany). The cuvette was incubated on ice for 5 minutes and was electroporated (parameters: charging voltage $1,500 \mathrm{~V}$, resistance $200 \Omega$ and capacitance $25 \mu \mathrm{F}$ ). Immediately after electroporation, one $\mathrm{ml}$ cold $1 \mathrm{M}$ sorbitol was added into cuvette and incubated in $30^{\circ} \mathrm{C}$ for 1 hour, and then plated on minimal media (Lin-Cereghino, J., Wong, W. W. et al., 2005).

\subsection{Reverse transcription real-time PCR}

The total RNA was isolated using the RNeasy Mini Kit (Qiagen) from cells that were isolated from mid log-phase (OD600 of $0.8-1.2$ ). The cells were broken using a mechanical disruption protocol with a Fast Prep 120 machine (RNeasy Mini Handbook page 49 -51) and the RNA concentration was measured using a Nanodrop spectrophotometer (NanoDrop Technologies Inc., Wilmington, Germany) (Qiagen, 2006; Bader, O., Schwarz, A. et al., 2012)

One $\mu \mathrm{g}$ of total RNA was treated with DNase I (Fermentas) to eliminate genomic DNA and the reaction was stopped by EDTA solution. The pure RNA was reverse transcribed to complementary DNA (cDNA) by using First Strand cDNA Synthesis Kit with the oligo (dT) 18 primer (Fermentas, 2009).

LightCycler ${ }^{\mathbb{R}}$ Real-time PCR from Roche system was used for measurement of the level of gene expression. The conditions for a $20 \mu 1$ reaction were as follows: $4 \mu 1$ of Master mix, $1 \mu 1$ of $20 \mu \mathrm{M}$ each primer, and $2 \mu 1$ of cDNA $(20 \mathrm{ng} / \mu 1) ; 95^{\circ} \mathrm{C}$ for 10 minutes, 45 cycles of $95^{\circ} \mathrm{C}$ for 10 seconds, $50^{\circ} \mathrm{C}$ for 10 seconds, $72^{\circ} \mathrm{C}$ for 10 seconds (Roche, 2007). The value of gene expression was normalized against the two housekeeping genes $A C T 1$ and SLT2 in all analyses. 


\subsection{Growth assay}

C. glabrata, C. albicans and S. cerevisiae cells were grown at $37^{\circ} \mathrm{C}$ for overnight in a shaking incubator. Cells from the overnight culture were harvested by centrifugastion, washed with $\mathrm{ddH}_{2} \mathrm{O}$ and adjusted to a density of $0.5 \mathrm{McF}$ arland $\left(5 \mathrm{X} 10^{6}\right.$ cells $\left./ \mathrm{ml}\right)$ in $0.85 \% \mathrm{NaCl}$. The adjusted cell suspension was diluted 10-folds ( $1 \mathrm{ml}$ cell suspension in $9 \mathrm{ml} 0.85 \% \mathrm{NaCl}$ ). $100 \mu \mathrm{l}$ of the diluted cell suspension was added into 96 well plates containing 2-fold concentrated RPMI1640 or YPD. For each strain, growth of three yeast suspensions were monitored at $30^{\circ} \mathrm{C}$ and $37^{\circ} \mathrm{C}$ by measuring the OD630 every 3 hours for 36 hours.

\subsection{Susceptibility testing to cell perturbing agents}

\subsubsection{Agar drop dilution assay}

Susceptibility testing to cell perturbing agents (Caffeine, Calcofluor white, and Congo red) was performed by agar drop dilution. To prepare the inoculum, yeast cells from agar plates were added to sterile $0.85 \% \mathrm{NaCl}$ to a density of $0.5 \mathrm{McF}$ arland $\left(5 \times 10^{6}\right.$ cells $\left./ \mathrm{ml}\right)$, diluted 10 -fold in sterile $0.85 \% \mathrm{NaCl}$. Five $\mu \mathrm{l}$ of the diluted cell suspension was dropped onto agar plate containing cell perturbing agents. The plates were incubated at $30^{\circ} \mathrm{C}$ for 24 hours in case of $C$. glabrata and 48 hours in case of C. albicans and S. cerevisiae

\subsubsection{Broth micro-dilution assay}

Susceptibility testing to cell wall perturbing agents (Calcofluor white, Congo red, Sodium dodecyl sulfate (SDS), Hygromycin B, Brefeldin A, Nikkomycin Z, and Lufenuron) was performed by broth micro-dilution. To prepare the inoculum, cells from agar plates were added to $0.85 \% \mathrm{NaCl}$ to a density of $0.5 \mathrm{McFarland}\left(5 \times 10^{6}\right.$ cells $\left./ \mathrm{ml}\right)$, diluted 10 -fold in $0.85 \% \mathrm{NaCl}$ and $100 \mu \mathrm{l}$ of the cell suspension was added to an equal volume of RPMI1640 containing the cell wall perturbing agent. 96 well plates with two-fold serial dilutions of cell wall perturbing agents were incubated at $37^{\circ} \mathrm{C}$ for 24 hours for C. glabrata and for 48 hours for S. cerevisiae and C. albicans. The cell densities were measured in a spectrophotometer at absorbance 630 (Dynex 
Technologies GmbH, Denkendorf, Germany). The minimum inhibitory concentration (MIC) 50 and MIC90 were calculated from the value of absorbance 630 in analogy to the EUCAST methodology (EUCAST, 2008).

\subsection{Alcian blue binding assay}

Alcian blue is a positively charged, blue colored dye, which binds to negative charges on the cell wall surface. The Alcian blue binding assay was adapted from two previous publications (Odani, T., Shimma, Y. et al., 1996; Hobson, R. P., Munro, C. A. et al., 2004). The overnight culture of yeast cells in YPD were adjusted to an OD600 of 1 in sterile PBS and centrifuged to collect the cells. The cell pellets were suspended in $1 \mathrm{ml}$ of $30 \mu \mathrm{g} / \mathrm{ml}$ Alcian Blue in $0.02 \mathrm{M} \mathrm{HCl}$ ( $\mathrm{pH}$ 3.0), incubated at room temperature for 10 minutes, and pelleted by centrifugation. $100 \mu \mathrm{l}$ of supernatant was transferred into 96 well plates to measure the absorbance $630 \mathrm{~nm}$ by using a spectrophotometer. Alcian blue concentrations were determined by reference to a standard curve. Alcian Blue binding was calculated according to the formula: $x=(u-v) \div n$ where $x=$ Alcian blue binding, $u=$ original Alcian blue concentration $(\mu \mathrm{g} / \mathrm{ml}), v=$ final Alcian blue concentration $(\mu \mathrm{g} / \mathrm{ml})$, and $n=$ amount of yeast cells in OD600.

\subsection{Flow cytometry measurement}

\subsubsection{Glucan staining assay}

Congo red was used to stain the glucan of the yeast cell wall. Cells from $1 \mathrm{ml}$ of an overnight culture were harvested and washed twice with PBS. Cells were resuspended in $1 \mathrm{ml}$ of $1 \mathrm{M} \mathrm{NaOH}$, boiled at $95^{\circ} \mathrm{C}$ for 5 minutes, washed again twice with PBS and incubated for 30 minutes at room temperature in $300 \mu \mathrm{l}$ Congo Red staining solution $(200 \mathrm{mg} / \mathrm{ml})$. After washing, stained cells were resuspended in $500 \mu \mathrm{l}$ of water (Bader, O., Schwarz, A. et al., 2012).

\subsubsection{Chitin staining assay}

Wheat-germ agglutinin (WGA-Alexafluor 647 conjugate (Invitrogen, OR, USA) was used to stain the chitin of the yeast cell wall. Cells from $1 \mathrm{ml}$ of an overnight culture were 
harvested and washed twice with PBS. Cells were resuspended in $1 \mathrm{ml}$ of $1 \mathrm{M} \mathrm{HCl}$, boiled at $95^{\circ} \mathrm{C}$ for 5 minutes and then washed again twice with PBS. Cells were resuspended in $300 \mu 1$ WGA staining solution $(50 \mu \mathrm{g} / \mathrm{ml})$, incubated for 30 minutes at room temperature and washed again twice. Stained cells were resuspended in $500 \mu \mathrm{l}$ water (Bader, O., Schwarz, A. et al., 2012).

After staining, flow cytometric analyses were performed with a FACS Calibur flow cytometer using Cell QuestPro software (BD-Biosciences, NJ, USA). All values were calculated as the mean average fluorescence intensity of 12,000 events in three independent experiments (Bader, O., Schwarz, A. et al., 2012).

\subsection{Biofilm properties determination}

\subsubsection{Hydrophobicity assay}

The overnight culture of yeast cells in YPD were washed with $\mathrm{ddH}_{2} \mathrm{O}$ and adjusted to OD600 of 1 in sterile water. Two $\mathrm{ml}$ of cell suspensions were mixed with $2 \mathrm{ml}$ Octane in glass tube with rubber cover and rigourously vortexed for 30 seconds. After the separation of the two layers, one $\mathrm{ml}$ of the bottom aqueous phase was taken to measure the OD600 by using spectrophotometer (de Groot, P. W., Kraneveld, E. A. et al., 2008). Hydrophobicity was calculated as the percentage of cells removed from the aqueous phase.

\subsubsection{Polystyrene adhesion assay}

Cells were grown in YPD for 24 hours, after that the OD600 was adjusted to 2, and $50 \mu 1$ of these cell suspensions were transferred into $150 \mu \mathrm{l}$ fresh YPD medium in polystyrene 96-wells plates (Greiner bio-one). After 24 hours of incubation at $37^{\circ} \mathrm{C}$, non-adherent cells were washed out with sterile water and the adherent cells were stained with $0.1 \%$ Crystal violet for 5 minutes. Excess Crystal violet was washed away and the cells were lysed with a solution containing 50\% ethanol and 1\% SDS. The amount of adherent cells was determined by measuring crystal violet released from the lysed cells as the absorbance at $630 \mathrm{~nm}$ (Bader, O., Schwarz, A. et al., 2012). 


\subsubsection{Silicone adhesion assay}

$1 \mathrm{x} 1 \mathrm{~cm}$. of silicone pieces (AMT Aromando, Duesseldorf, Germany) were incubated for 24 hours at $37^{\circ} \mathrm{C}$ in 12-wells plates containing $2 \mathrm{ml}$ YPD inoculated at a cell density of 0.08 McFarland with the C.glabrata strains. To remove unbound cells by aspiration, silicone pieces were transferred into fresh 12-wells plates containing $1 \mathrm{ml}$ PBS. Bound cells were scraped off, resuspended in $1.2 \mathrm{ml} \mathrm{PBS}$, and quantified by measuring the OD600 (Bader, O., Schwarz, A. et al., 2012).

\subsection{Protein expression in $P$. pastoris}

Ecm33 and Pst1 protein expression in Pichia pastoris was performed using the EasySelectTM Pichia Expression Kit (Invitrogen, CA, USA). Both proteins were expressed without the C-terminal signal peptides and $\omega$-sites of the $\mathrm{N}$-terminal regions.

The inserted fragments of ECM33 and PST1 were amplified from C. glabrata CBS138/ATCC2001 genomic DNA with specific primers including adaptors for restriction enzyme sites. A $50 \mu 1$ reaction $(5 \mu 1$ of $10 x$ KOD Taq buffer, $5 \mu 1$ of $2 \mathrm{mM}$ dNTPs, $3 \mu 1$ of 25 $\mathrm{MgCl}_{2}, 1 \mu \mathrm{l}$ of $20 \mu \mathrm{M}$ each primer, $1 \mu \mathrm{l}$ of KOD Taq-Polymerase (1U/ $\mu 1$; Novagen), and $2 \mu 1$ of genomic DNA $(50 \mathrm{ng} / \mu \mathrm{l}))$ was subjected to cycling $\left(95^{\circ} \mathrm{C}\right.$ for 5 minutes, 35 cycles $95^{\circ} \mathrm{C}$ for 30 seconds, $58^{\circ} \mathrm{C}$ for 30 seconds, $70^{\circ} \mathrm{C}$ for 30 seconds, finally 5 minutes at $70^{\circ} \mathrm{C}$ ) in a thermocycler machine. The expected PCR product size of each gene is shown in Table 10. The PCR product of target gene will be inserted at multiple cloning site of pPICZ $\alpha$ vector as shown in Figure 7.

The pPICZ $\alpha$ and the inserted fragments were digested with restriction enzyme (EcoRI and KpnI for ECM33 and EcoRI and XbaI for PST1) and the modified fragments and pPICZ $\alpha$ linearlized plasmid purified using the QIAquick Gel Extraction Kit (Qiagen). The ligation of the modified fragments and pBM51 linearlized plasmid were ligated by using T4 DNA ligase (Thermo Scientific) as follows: linear vector $50 \mathrm{ng}$, inserted DNA $250 \mathrm{ng}, 2 \mu \mathrm{l}$ of 10X T4 DNA ligase buffer and $1 \mu \mathrm{l}$ of T4 DNA ligase (1U/ $\mu 1$ of). The mixtures were incubated at $25^{\circ} \mathrm{C}$ for 1 hour and kept in $4{ }^{\circ} \mathrm{C}$ for overnight. A $5 \mu$ of the mixture was used for transformation in the competent DH5 $\alpha$ E. coli with heat schock transformation protocol (see the detail protocol in 
2.10.1 and 2.10.2). The transformant DH5 $\alpha$ E. coli was selected by using Zeocin ${ }^{\mathrm{TM}}$ (Invitrogen, CA, USA) drug resistance.

Positive DH5 $\alpha$ clones were verified by restriction enzyme double digestion and DNA sequencing. The pure recombinant pPICZ $\alpha$ no. P4 and P5 were extracted and used to transform methylotrophic P. pastoris by electroporation (see the detail protocol in 2.10.3 and 2.10.4). The transformant $P$. pastoris was selected by using YPD agar containing Zeocin ${ }^{\mathrm{TM}}$. The recombinant $P$. pastoris can grow in this selective YPD agar because it contains Zeocin resistant gene.

\subsubsection{Methanol induced Ecm33 and Pst1 protein expression}

ECM33 and PST1 genes were transformed to the P. pastoris strain X33 (wid-type) and GS115 (his4). For expression, a representative single colony of each of the four recombinant strains (GS115:CgECM33, GS115:CgPST1, X33:CgECM33 and X33:CgPST1) was grown in 25 $\mathrm{ml} \mathrm{BMGH} \mathrm{(buffered} \mathrm{minimal} \mathrm{glycerol} \mathrm{histidine)} \mathrm{in} \mathrm{a} 250 \mathrm{ml}$ flask and incubated at $30^{\circ} \mathrm{C}$ for overnight that these cells will be in log-phase growth. These cells were harvested by centrifugation, resuspended and adjusted to an OD600 of 1 in $100 \mathrm{ml} \mathrm{BMMH} \mathrm{(buffered} \mathrm{minimal}$ methanol histidine) media in $500 \mathrm{ml}$ flask. Then, the cell suspensions were incubated at $30^{\circ} \mathrm{C}$ in shaking incubator $250 \mathrm{rpm}$ for induction of ECM33 and PST1 expression.

One $\mathrm{ml}$ of cell suspensions were collected at 24, 48, 72, 96, and 120 hours to determine the time points that express the highest amounts of proteins in the four recombinant strains by using SDS-PAGE (Invitrogen, 2009).

\subsubsection{SDS-Polyacrylamide gel electrophoresis}

All components of separating gel were mixed and pipetted into assembled gel plates of a BioRad Mini Protean II system. Then, $100 \mu \mathrm{l}$ of butanol was added onto the top of gel to make a straight top of separating gel and the gel allowed to polymerize. The gel was cleaned by removing butanol and washing with water. All components of collective gel were mixed and pipetted onto the separating gel and that the comb was put into the gel and the gel allowed to polymerize (SDS-PAGE gel preparation is shown in Table 11). SDS-PAGE gels were stored at $4^{\circ} \mathrm{C}$ in plastic bag until use. 
The protein solution was mixed with $5 \mathrm{X}$ loading buffer and boiled at $95^{\circ} \mathrm{C}$ for 10 minutes then loaded onto the SDS-PAGE gel. Coomassie blue was used to stain the proteins in the SDSPAGE gels.

Table 10. Generation of recombinant ECM33 and PST1 plasmids for protein expression

\begin{tabular}{ccccc}
\hline Gene & Size & Primer code & $\begin{array}{c}\text { Annealing temp. } \\
(\mathrm{bp})\end{array}$ & $\begin{array}{c}\text { Restriction } \\
\text { enzyme }\end{array}$ \\
\hline$C g E C M 33^{\circ}$ & 1,150 & 1 and 2 & 58 & EcoR $\mathrm{I} /$ Kpn $\mathrm{I}$ \\
$C g P S T 1^{1}$ & 1,183 & 3 and 7 & 58 & EcoRI $/$ Xba I \\
$C g E C M 33^{2}$ & 1,146 & 17 and 18 & 58 & BamHI $/$ Pst $\mathrm{I}$ \\
\hline
\end{tabular}

${ }^{1}$ : protein expression in $P$. pastoris.

2: protein expression in E. coli.

Table 11. SDS-PAGE gel preparation

\begin{tabular}{l|c|c|c|c}
\hline & Collective gel & \multicolumn{3}{|c}{ Separating gel } \\
\cline { 2 - 5 } & $5 \%$ & $10 \%$ & $12 \%$ & $15 \%$ \\
\hline Water & $1.4 \mathrm{ml}$ & $2.95 \mathrm{ml}$ & $2.45 \mathrm{ml}$ & $1.7 \mathrm{ml}$ \\
Buffer & $630 \mu 1$ & $1.9 \mathrm{ml}$ & $1.9 \mathrm{ml}$ & $1.9 \mathrm{ml}$ \\
Acrylamide solution $^{\mathrm{a}}$ & $415 \mu \mathrm{l}$ & $2.5 \mathrm{ml}$ & $3 \mathrm{ml}$ & $3.75 \mathrm{ml}$ \\
$10 \%$ SDS & $25 \mu \mathrm{l}$ & $75 \mu 1$ & $75 \mu \mathrm{l}$ & $75 \mu \mathrm{l}$ \\
$10 \%$ APS & $25 \mu \mathrm{l}$ & $75 \mu 1$ & $75 \mu 1$ & $75 \mu 1$ \\
TEMED & $2.5 \mu 1$ & $3 \mu 1$ & $3 \mu 1$ & $3 \mu 1$ \\
\hline
\end{tabular}

${ }^{a}: 30 \%$ acrylamide and bisacrylamide stock solution at a ratio of 37.5:1 


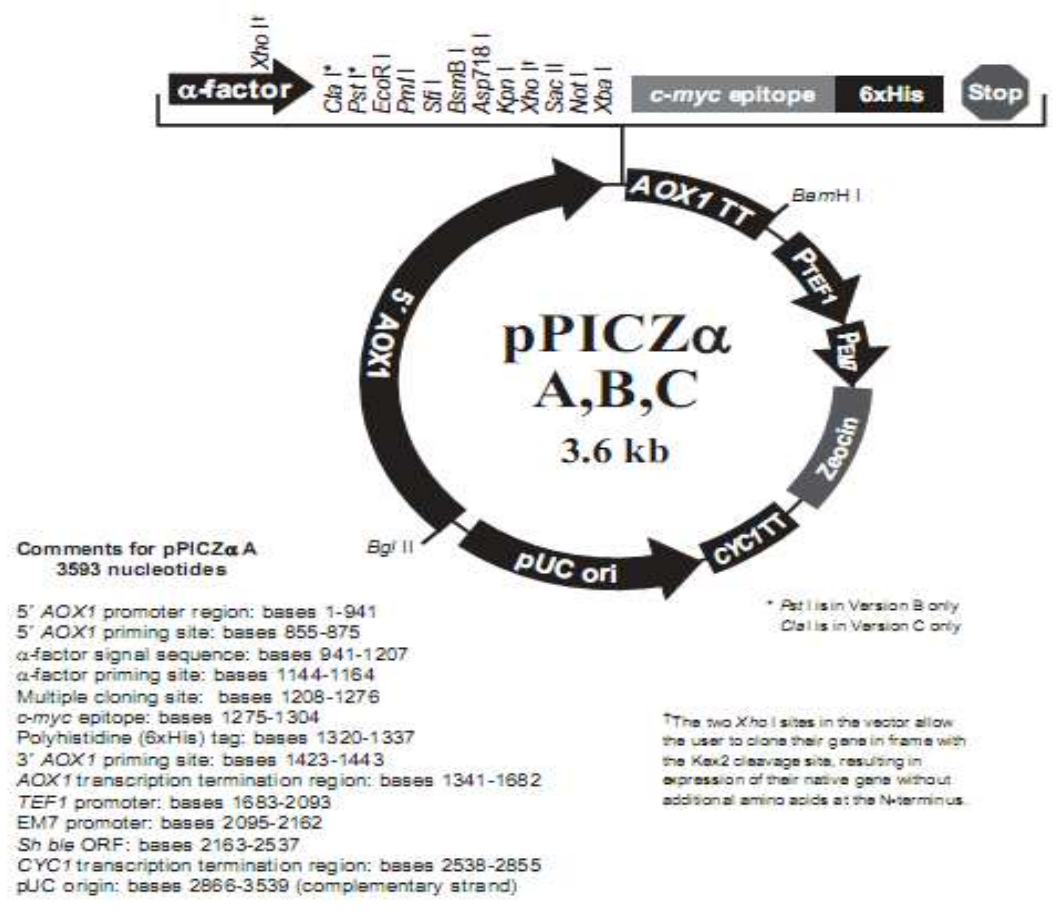

Figure 7. Map of pPICZa

pPIC $\alpha$ plasmid is 3.6 kilobases vector that used to express and secrete recombinant proteins in $P$. pastoris. Recombinant protein is expressed as fusions to an N-terminal peptide encoding the $S$. cerevisiae $\alpha$-factor secretion signal. This plasmid allows high-level, methanol inducible expression of the gene of interest in P. pastoris. pPIC $\alpha$ plasmid contains the following elements: AOX1 promoter for methanol-induced expression of the gene of interest, $\alpha$-factor secretion signal for directing secreted recombinant protein expression, Zeocin resistance gene for selection, C-terminal peptide containing the c-myc epitope and a polyhistidine (6xHis) tag for detection and purification. Figure taken from (Invitrogen, 2009).

\subsection{Protein deglycosylation}

N-glycosidase (Endo- $\alpha-\mathrm{N}$-Acetylgalactosaminidase; New England BioLabs Inc.) and Oglycosidase (PNGase F; New England BioLabs Inc.) were used to be a tool to eliminate Nglycan and O-glycan, respectively. The mixture of substrate and glycosidase enzyme was incubated at $37^{\circ} \mathrm{C}$ for 2 hours according to the manufacturer's instructions. 


\subsection{Protein expression in $E$. coli and Anti-Ecm33 antibody production}

\subsubsection{Cloning of ECM33 gene in $E$. coli}

F-LM-pQE and R-LM-pQE primers (Table 8) were used to amplify the ECM33 gene of C. glabrata without the signal peptide and the GPI-anchoring site. A $50 \mu 1$ reaction $(5 \mu 1$ of 10x KOD Taq buffer, $5 \mu 1$ of $2 \mathrm{mM}$ dNTPs, $3 \mu 1$ of $25 \mathrm{MgCl} 2,1 \mu 1$ of $20 \mu \mathrm{M}$ each primer, $1 \mu 1$ of KOD Taq-Polymerase (1U/ $\mu \mathrm{l}$; Novagen) and $2 \mu \mathrm{l}$ of genomic DNA $(50 \mathrm{ng} / \mu \mathrm{l}))$ was subjected to cycling $\left(95^{\circ} \mathrm{C}\right.$ for 5 minutes, 35 cycles of $95^{\circ} \mathrm{C}$ for 30 second, $58^{\circ} \mathrm{C}$ for 30 second, $70^{\circ} \mathrm{C}$ for 30 second, finally 5 minutes at $70^{\circ} \mathrm{C}$.) in a thermocycler machine.

The PCR product of ECM33 (product size 1,146 bp) was cloned into the pQE30 expression vector (Figure 8) by using BamHI and PstI restriction enzymes as shown in Table 10. The recombinant plasmid pQE30:CgECM33 was transformed to the E. coli strain DH5 $\alpha$ for plasmid multiplication and BL-21 for Ecm33 protein expression. Ampicillin resistance was used to select the positive clones (Qiagen, 2003). The detail of the ligation, E. coli competent preparation, and transformation were described in 2.10.1 and 2.10.2.

The positive DH5 $\alpha$ clones were verified by using restriction enzyme double digestion and DNA sequencing.

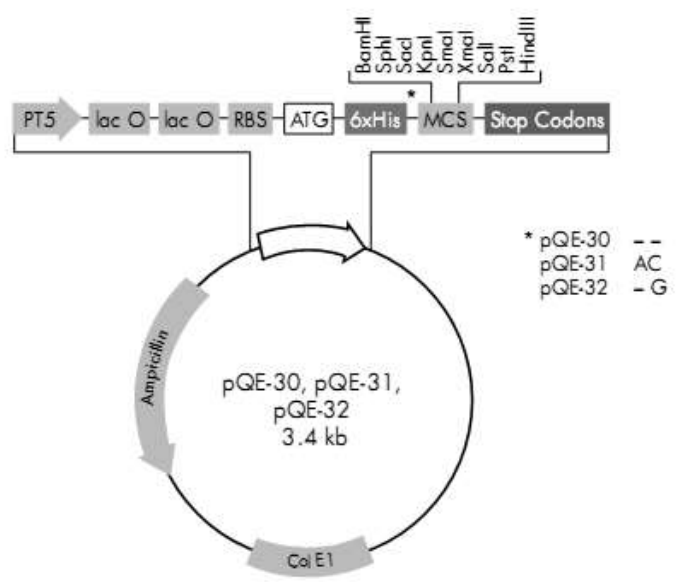

Figure 8. Map of pQE-30

pQE-30 plasmid is 3.4 kilobases vector that used to express recombinant protein in E. coli. This vector contains the following elements: the phage T5 promoter transcription-translation system, two lac operator sequences, 6xHis-tag coding sequence, multiple cloning site, $\beta$-lactamase gene $(B L A)$ conferring resistance to ampicillin, and ColE1 origin of replication (Qiagen, 2003). 


\subsection{2 $C g E \mathrm{Em} 33$ protein expression in $E$. coli}

A single colony of recombinant E. coli was grown in $20 \mathrm{ml}$ of LB broth containing 100 $\mu \mathrm{g} / \mathrm{ml}$ ampicillin and $25 \mu \mathrm{g} / \mathrm{ml}$ of kanamycin at $37^{\circ} \mathrm{C}$ for overnight in shaker incubator at 250 rpm. The overnight culture was diluted 50-folds (4 $\mathrm{ml}$ in $200 \mathrm{ml} \mathrm{LB}$ with antibiotics) and grown at $37^{\circ} \mathrm{C}$ with vigorous shaking until an OD600 of 0.6-0.8 was reached. IPTG (Roth) was added into the culture medium with a final concentration $1 \mathrm{mM}$ and incubated for 4-5 hours. The Ecm33 expressing cells were harvested by centrifugation at 1,942 x g (3,000 rpm) for 10 minutes and frozen in $-80^{\circ} \mathrm{C}$ (Qiagen, 2003).

\subsubsection{Ecm33 protein purification}

The pellect from $50 \mathrm{ml}$ culture was mixed with lysis solution for protein expression $(5 \mathrm{ml}$ of Bugbuster $^{\circledR}$ (Merck), $0.1 \mathrm{mg}$ of lysozyme powder (Fluka), $5 \mu \mathrm{l}$ of Benzouase (Novagen), and $220 \mu \mathrm{l}$ of protease inhibitor cocltail (Roche) and incubated for 40 minutes at room temperature. After incubation, the cloudy solution was centrifuged to collect the pellet and dissolved with $8 \mathrm{M}$ urea solution at $40^{\circ} \mathrm{C}$ for 20 minutes. The protein solution was centrifuged at $1,942 \times \mathrm{g}(3,000$ $\mathrm{rpm})$ to remove the cell debris and the supernatant was kept at $-20^{\circ} \mathrm{C}$.

His-Tag affinity chromatography was used to purify the Ecm33 protein. Two $\mathrm{ml}$ of NiNTA resin solution (Qiagen) was added into a column. Columns were equilibrated with $5 \mathrm{CV}$ wash buffer. The lysate was loaded into column and the flow-through was collected in 3 portions of $1 \mathrm{ml}$. The target protein that bound to Ni-NTA resin was washed with $10 \mathrm{CV}$ wash buffer and the flow-through was collected in 5 portions of $1 \mathrm{ml}$. The target protein was eluted with wash buffer containing $250 \mathrm{mM}$ imidazole, and collected in 5 portions of $1 \mathrm{ml}$. All samples were analyzed by using SDS-PAGE (see the detail in 2.17.2).

\subsubsection{Protein concentration measurement}

BIOQUANT $^{\circledR}$ (Merck) was used to measure the concentration of protein in a solution according to the manufacturer's instructions. $0.05-0.5 \mathrm{mg} / \mathrm{ml}$ of Bovine serum albumin (BSA) was used to make a calibration curve. The protein solution was mixed with $200 \mu 1$ of 
BIOQUANT $^{\circledR}$, incubated for 2 minutes at room temperature and measured absorbance at 570 nm.

\subsubsection{Western blotting analysis}

Protein from SDS-PAGE was transferred onto a PVDF membrane by using wet blotting. After blotting, the PVDF membrane was blocked with $20 \mathrm{ml}$ of 5\% (w/v) skim milk for 1 hour and then washed twice with $20 \mathrm{ml}$ of PBST. The PVDF membrane was incubated with the primary antibody at $4^{\circ} \mathrm{C}$ for overnight and then washed three times with $20 \mathrm{ml}$ of PBST. The secondary antibody was added and incubated at room temperature for 2 hours and the membrane washed three times with $20 \mathrm{ml}$ of PBST. ECL ${ }^{\text {тм }}$ Western blotting Detection Reagent (GE Healthcare, Buckinghamshire, UK) was used in the detection step and the image was taken with the Fujifilm LAS 4000 system.

\subsubsection{Anti-Ecm33 antibody production}

The immunisation procedure was performed by Eurogentec, Liège, Belgium. Before start the immunization program, serum of non-immunized rabbits were tested for Ecm33 antibodies to circumvent cross-reactions.

A polyclonal anti-Ecm33 serum was produced with the "anti-protein 87-days polyclonal package" of Eurogentec. Briefly, $100 \mu \mathrm{g}$ purified $C g E \mathrm{~cm} 33$ protein antigen was immunized in a rabbit on day $0,14,28$, and 56 . Serum was taken from the rabbits at day 0 (pre-immune serum) and day 38, 66, and 87 after immunization as shown in Figure 9. The immunized rabbit serum was tested for $C g E c m 33$ antigen - anti-Ecm33 polyclonal antibody reaction by using immunoblotting.

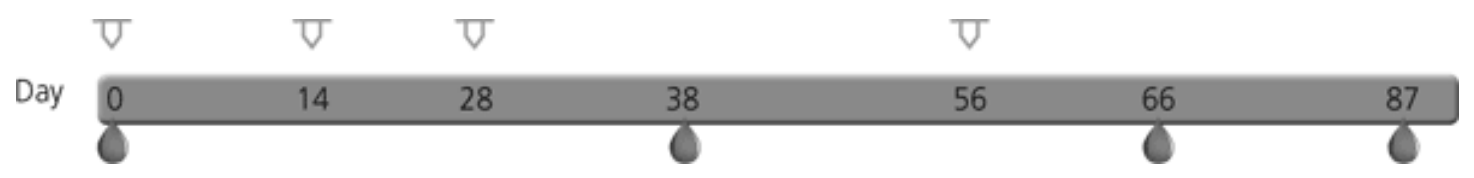

Figure 9. The time line of the anti-Ecm33 antibody production

Figure taken from https://secure.eurogentec.com/product/research-anti-protein-87-daypolyclonal-packages.html 


\subsection{Cell wall protein release assay}

An overnight culture of yeast cells in YPD was washed and adjusted to an OD600 of 0.1 in $100 \mathrm{ml}$ of minimum medium (MM). The cell suspension was incubated at $37^{\circ} \mathrm{C}$ for 24 hours in shaker incubation at $250 \mathrm{rpm}$. The supernatant was collected by two times of centrifugation at $486 \mathrm{x} \mathrm{g}(1,500 \mathrm{rpm})$ for 10 minutes to remove the cells. To concentrate the supernatant, a $15 \mathrm{ml}$ spin column with a pore size of $10 \mathrm{kDa}$ (Sartorius, Göttingen) was used to reduce the volume of supernatant 50 -folds. The protein concentration in the supernatant was determined by using BIOQUANT $^{\circledR}$ (see the detail in 2.19.4). Western blot analyses were performed to check for reactivity of released material with rabbit anti-Cwp1 or rabbit anti-1,6- $\beta$-glucan.

\subsection{Cell wall extraction}

Cell wall extraction was performed as described de Groot et al (de Groot, P. W., de Boer, A. D. et al., 2004; de Groot, P. W., Kraneveld, E. A. et al., 2008). Briefly, yeast cells were fully disintegrated with 0.25 to $0.50 \mathrm{~mm}$. glass beads (Emergo BV) in the presence of a protease inhibitor cocktail (Sigma) using a Bio-Savant FastPrep 120 machine (Qbiogene). To remove non-covalently linked proteins and intracellular contaminants, isolated cell walls were washed extensively with $1 \mathrm{M} \mathrm{NaCl}$ and twice extracted with $2 \% \mathrm{SDS}, 150 \mathrm{mM} \mathrm{NaCl}, 100 \mathrm{mM} \mathrm{Na}-$ EDTA, $100 \mathrm{mM} \beta$-mercaptoethanol, and $50 \mathrm{mM}$ Tris-HCl, $\mathrm{pH} \mathrm{7.8,} \mathrm{for} 5$ minutes at $100^{\circ} \mathrm{C}$. SDStreated walls were washed with water until no foaming was observed anymore. The extracted cell walls (ECWs) were freeze-dried with lyophilization and stored at $20^{\circ} \mathrm{C}$ until further use.

\subsection{Binding assay}

$20 \mu 1(20 \mathrm{mg} / \mathrm{ml})$ of extracted cell walls (ECWs) were incubated with $20 \mu \mathrm{l}$ of ELISA blocking solution for 1 hour at $37^{\circ} \mathrm{C}$ and washed twice with $1 \mathrm{ml}$ of PBS. The blocked ECWs were incubated with $10 \mu \mathrm{l}(250 \mu \mathrm{g} / \mathrm{ml})$ of Ecm33 or Pst1 protein for 1 hour at $37^{\circ} \mathrm{C}$ and washed twice with $1 \mathrm{ml}$ of PBS to remove unbound protein. The pellets were resuspended with $10 \mu \mathrm{l}$ steriled water and $4 \mu 1$ of $5 \mathrm{X}$ loading buffer. Potential binding between ECWs and recombinant protein was analyzed by western blot analysis. 


\subsection{Statistical analyses}

Three independent experiments were done in all yeast strains. Statistical significance for phenotypic tests was calculated using Student's t-test with Microsoft Excel. $p$-value $<0.05$ were considered to be significant. 


\section{CHAPTER 3}

\section{RESULTS}

\subsection{In silico analysis of the Ecm33 family}

In silico analyses showed that the Ecm33, Pst1, Sps2 and Sps22 proteins of C. glabrata consist of a signal peptide, and a short serine/threonine rich region, preceeding an omega site for GPI anchor addition at the C-terminus. Framing the central part of the mature protein four conserved cysteine sites can be found, potentially adding to the protein stability by formation of disulphide bonds as shown in Figure 10 and Figure 11.

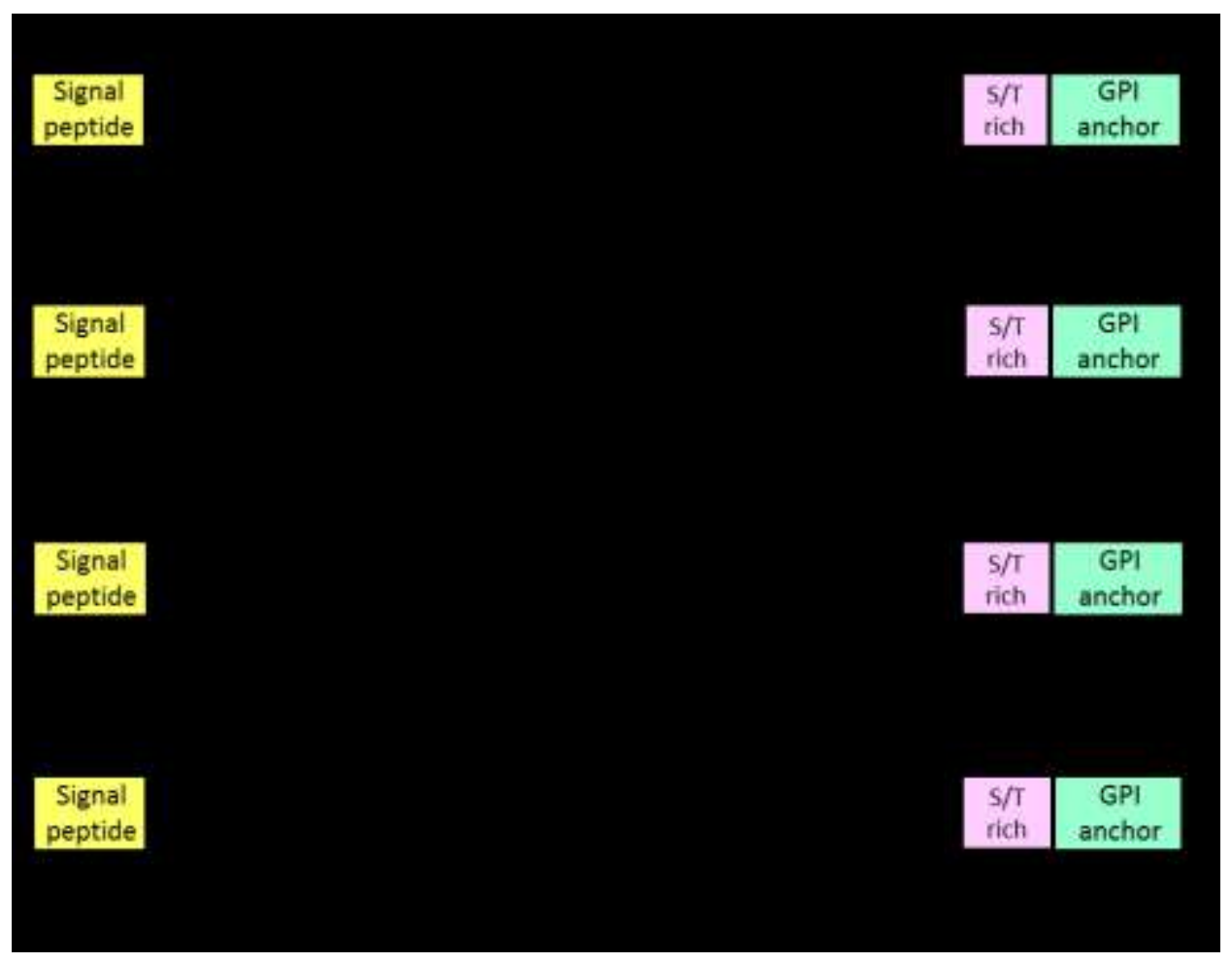

Figure 10. Ecm33 protein family structure of $C$. glabrata

Signal peptides were predicted by using SignalP. Omega sites for GPI anchor attachment were predicted using the big-PI Predictor server. All proteins feature a serine/threonine rich region and four conserved cysteine residues, potentially involved in the formation of disulfide bonds. Residue numbers are given in reference to each individual protein sequence. 


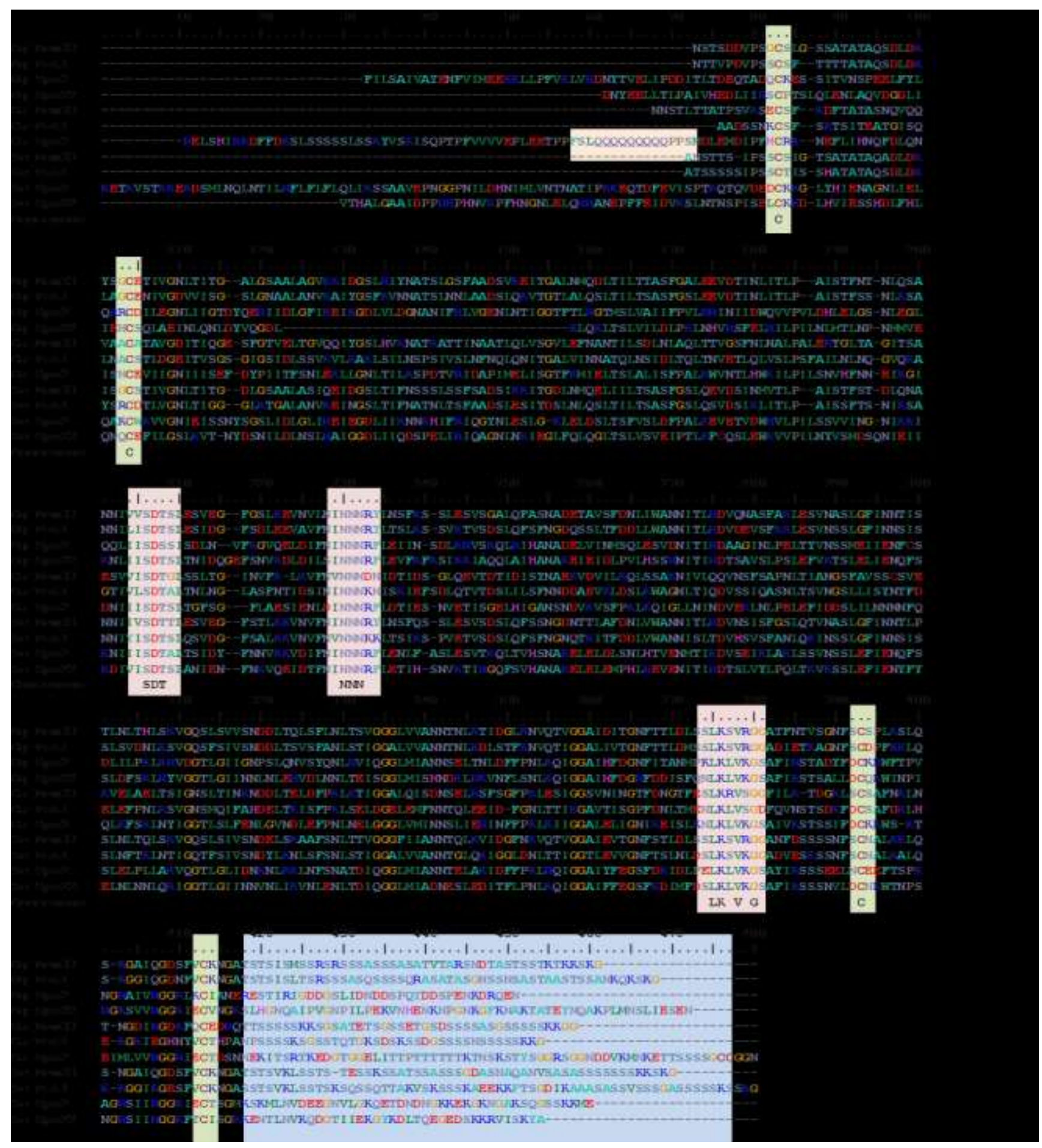

Figure 11. Sequence alignment of mature proteins of the Ecm33 families in $C$. glabrata, $C$. albicans and $S$. cerevisiae.

Shaded regions; green: conserved cysteins, red: fully conserved residues, potentially contributing towards function, blue: S/T-rich region. Signal peptides for ER-entry and GPI-modification were removed from the sequences before alignment. 
The relatedness of the different proteins of the Ecm33 families of C. glabrata, C. albicans, and S. cerevisiae were analysed by creation of a protein sequence alignment and a phylogenetic tree derived thereof.

Next to the four conserved cysteines already described above for the four proteins from C. glabrata, three additional highly conserved regions can be observed in the mature protein sequences: at positions 206-208 an SDT motif, at postions 230-232 an NNN motif and last at positions 375-380 a LK.V.G-motif as shown in Figure 11. Other than that, several conserved proline and glycine residues are found.

Amino acid identity matrices (Table 12 and Table 13) show that C. glabrata Ecm33 shares $67 \%$ homology with the Ecm 33 protein of $S$. cerevisiae and $73 \%$ in the Pst1 protein. In contrast, the homology to the respective proteins in C. albicans is only $30 \%$ and $33 \%$, respectively. Although Sps2 and Sps22 are grouped in the same family, the homology with the Ecm 33 protein is only approximately 30\% in all three organisms. Phylogenic clustering (Figure 12) and calculation of amino acid identity matrices indicates that the members of the Ecm 33 protein family in can be divided into two groups; (i) Ecm33 and Pst1, and (ii) Sps2 and Sps22. An ortholog of Sps22 is not found in C. albicans.

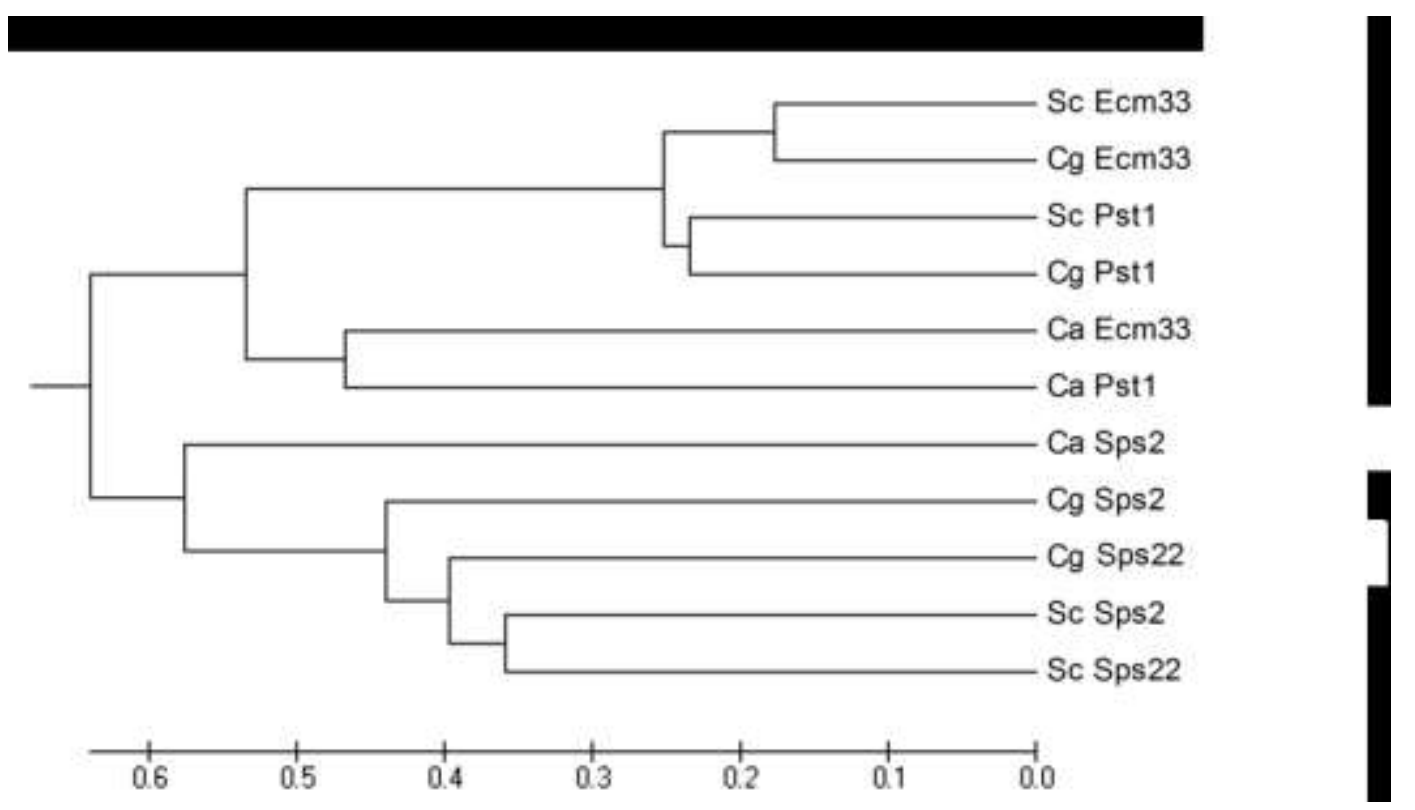

Figure 12. The Ecm33 family phylogeny of $C$. glabrata, $C$. albicans and $S$. cerevisiae.

Phylogenetic clustering of eleven protein sequences of the Ecm33 family in C. glabrata, $C$. albicans and $S$. cerevisiae shows separation into two distinct subgroups. An ortholog of the Sps22 protein is not found in the genome of $C$. albicans. 
Table 12. Identity matrix of the entire protein in the Ecm33 family of $C$. glabrata, $C$.

albicans and S. cerevisiae

\begin{tabular}{|l|c|c|c|c|c|c|c|c|c|c|c|}
\hline & Cg-Ecm33 & Cg-Pst1 & Cg-Sps2 & Cg-Sps22 & Ca-Ecm33 & Ca-Pst1 & Ca-Sps2 & Sc-Ecm33 & Sc-Pst1 & Sc-Sps2 & Sc-Sps22 \\
\hline Cg-Ecm33 & ID & 0.62 & 0.196 & 0.213 & 0.308 & 0.317 & 0.213 & 0.67 & 0.524 & 0.24 & 0.216 \\
\hline Cg-Pst1 & 0.62 & ID & 0.214 & 0.213 & 0.327 & 0.315 & 0.203 & 0.561 & 0.556 & 0.235 & 0.225 \\
\hline Cg-Sps2 & 0.196 & 0.214 & ID & 0.313 & 0.177 & 0.213 & 0.21 & 0.197 & 0.2 & 0.324 & 0.355 \\
\hline Cg-Sps22 & 0.213 & 0.213 & 0.313 & ID & 0.201 & 0.193 & 0.224 & 0.2 & 0.196 & 0.304 & 0.347 \\
\hline Ca-Ecm33 & 0.308 & 0.327 & 0.177 & 0.201 & ID & 0.366 & 0.174 & 0.331 & 0.31 & 0.205 & 0.19 \\
\hline Ca-Pst1 & 0.317 & 0.315 & 0.213 & 0.193 & 0.366 & ID & 0.213 & 0.315 & 0.283 & 0.215 & 0.205 \\
\hline Ca-Sps2 & 0.213 & 0.203 & 0.21 & 0.224 & 0.174 & 0.213 & ID & 0.213 & 0.24 & 0.237 & 0.253 \\
\hline Sc-Ecm33 & 0.67 & 0.561 & 0.197 & 0.2 & 0.331 & 0.315 & 0.213 & ID & 0.572 & 0.252 & 0.238 \\
\hline Sc-Pst1 & 0.524 & 0.556 & 0.2 & 0.196 & 0.31 & 0.283 & 0.24 & 0.572 & ID & 0.233 & 0.229 \\
\hline Sc-Sps2 & 0.24 & 0.235 & 0.324 & 0.304 & 0.205 & 0.215 & 0.237 & 0.252 & 0.233 & ID & 0.4 \\
\hline Sc-Sps22 & 0.216 & 0.225 & 0.355 & 0.347 & 0.19 & 0.205 & 0.253 & 0.238 & 0.229 & 0.4 & ID \\
\hline
\end{tabular}

ID: identity

Table 13. Identity matrix of Ecm33 family effector domains only of $C$. glabrata, $C$. albicans

and $S$. cerevisiae

\begin{tabular}{|l|c|c|c|c|c|c|c|c|c|c|c|}
\hline & Cg-Ecm33 & Cg-Pst1 & Cg-Sps2 & Cg-Sps22 & Ca-Ecm33 & Ca-Pst1 & Ca-Sps2 & Sc-Ecm33 & Sc-Pst1 & Sc-Sps2 & Sc-Sps22 \\
\hline Cg-Ecm33 & ID & 0.674 & 0.278 & 0.271 & 0.334 & 0.351 & 0.306 & 0.73 & 0.613 & 0.339 & 0.293 \\
\hline Cg-Pst1 & 0.674 & ID & 0.297 & 0.271 & 0.362 & 0.358 & 0.279 & 0.616 & 0.647 & 0.328 & 0.303 \\
\hline Cg-Sps2 & 0.278 & 0.297 & ID & 0.371 & 0.225 & 0.273 & 0.303 & 0.272 & 0.287 & 0.445 & 0.453 \\
\hline Cg-Sps22 & 0.271 & 0.271 & 0.371 & ID & 0.24 & 0.243 & 0.334 & 0.256 & 0.265 & 0.425 & 0.449 \\
\hline Ca-Ecm33 & 0.334 & 0.362 & 0.225 & 0.24 & ID & 0.37 & 0.231 & 0.325 & 0.352 & 0.268 & 0.237 \\
\hline Ca-Pst1 & 0.351 & 0.358 & 0.273 & 0.243 & 0.37 & ID & 0.296 & 0.342 & 0.333 & 0.304 & 0.267 \\
\hline Ca-Sps2 & 0.306 & 0.279 & 0.303 & 0.334 & 0.231 & 0.296 & ID & 0.293 & 0.327 & 0.343 & 0.366 \\
\hline Sc-Ecm33 & 0.73 & 0.616 & 0.272 & 0.256 & 0.325 & 0.342 & 0.293 & ID & 0.622 & 0.342 & 0.302 \\
\hline Sc-Pst1 & 0.613 & 0.647 & 0.287 & 0.265 & 0.352 & 0.333 & 0.327 & 0.622 & ID & 0.324 & 0.305 \\
\hline Sc-Sps2 & 0.339 & 0.328 & 0.445 & 0.425 & 0.268 & 0.304 & 0.343 & 0.342 & 0.324 & ID & 0.506 \\
\hline Sc-Sps22 & 0.293 & 0.303 & 0.453 & 0.449 & 0.237 & 0.267 & 0.366 & 0.302 & 0.305 & 0.506 & ID \\
\hline
\end{tabular}

ID: identity

\subsection{Confirmation of knock out strains}

Single deletion mutants of PST1 (Apst1) and ECM33 (Aecm33) and a double mutant (4pst1/4ecm33) in C. glabrata were provided by Tobias Schwarzmüller (Univerity of Vienna, 
Austria). There, the strains were generated by using a fusion PCR to construct a recombinant pTS50 plasmid that was used to transformed the parental strain (wild-type auxotroph $\Delta h t$ ), with selection for $N A T$ (nourseothricin-resistance gene) transformants on YPD medium plates (Schwarzmüller, T., 2009).

To comfirm the gene deletion status of the mutants received, the $4 p s t 1$ strain was analysed by using two PCR reactions: the first analysis using primers 13 F-MuE-Cont and 11 hk2 to verify integration of NAT gene at the 5' site and the second analysis using the primers 14 R-MuE-Cont and $12 \mathrm{hk} 3$ to confirm integration of the knock out cassette at the 3' end. Similarly, the deletion strain $\triangle e c m 33$ was tested with primer pair $15 \mathrm{~F}-\mathrm{MuM}$-Cont and $11 \mathrm{hk} 2$ for 5' site and primer pair $16 \mathrm{R}-\mathrm{MuM}$-Cont and $12 \mathrm{hk} 3$ for the 3' end. In strain $\Delta p s t 1 / \Delta e c m 33$, the primer pair $13 \mathrm{~F}-\mathrm{MuE}-\mathrm{Cont}$ and $11 \mathrm{hk} 2$ were used to confirm PST1 gene deletion and primer $16 \mathrm{R}-$ MuM-Cont and $12 \mathrm{hk} 3$ for ECM33 gene deletion. The PCR product size observed was approximately $800 \mathrm{bp}$ for all reactions as shown in Figure 13 as expected for positive reactions.

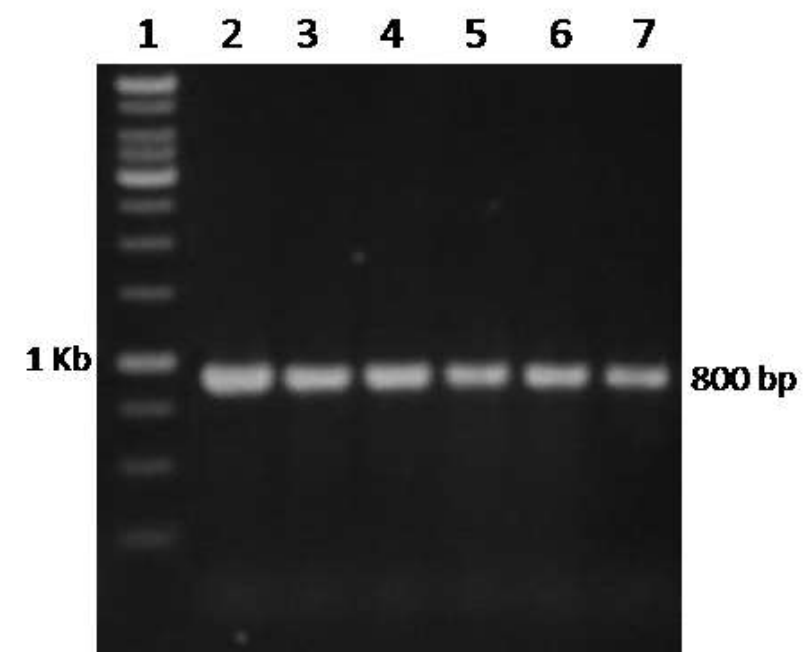

Figure 13. Confirmation of knock out strains by PCR

Lane 1: DNA Marker, lane 2: $\Delta p s t 1$ DNA with primer no. 13 and 11, lane 3: $\Delta p s t 1$ DNA with primer no. 14 and 12, lane 4: $\Delta e c m 33$ DNA with primer no. 15 and 11, lane 5: $\Delta e c m 33 D N A$ with primer no. 16 and 12, lane 6: Apst1:Aecm33 DNA with primer no. 13 and 11, and lane 7: Apst1:Aecm33 DNA with primer no. 16 and 12

As a further control, reverse transcription real-time PCR was performed to analyze gene expression in the generated mutant strains $\Delta p s t 1, \Delta e c m 33$ and $\Delta p s t 1 / \Delta e c m 33$ in comparison to the auxotroph parental strain $\Delta h t l$. In these assays, the housekeeping genes $A C T$ and $S L T$ were 
used for normalization of expression levels. The results confirmed that there was no expression of the deleted genes in the generated knock out strains. Furthermore, no compensatory upregulation of other family member genes was observed in the gene deletion mutants (Figure 14).

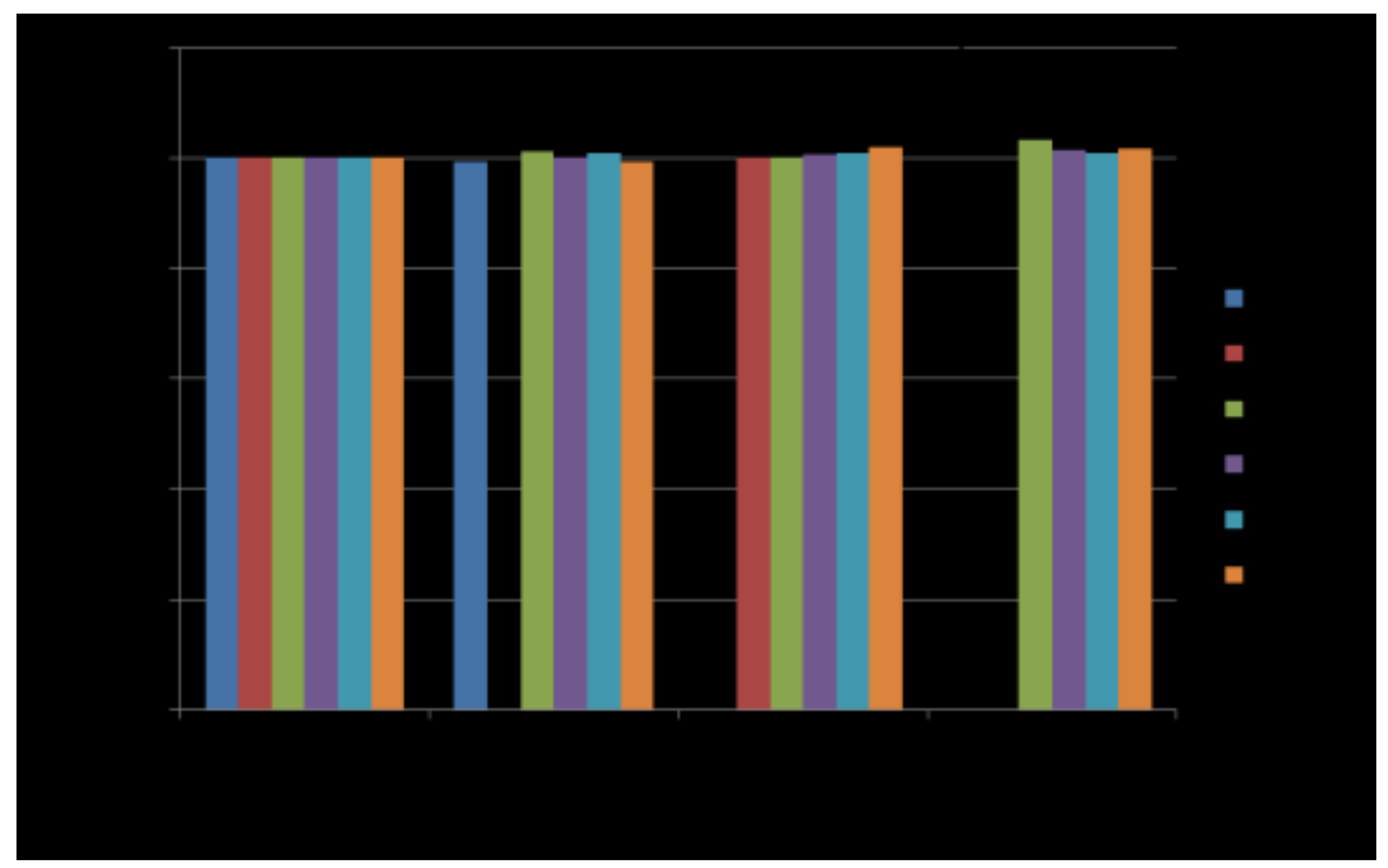

Figure 14. Reverse transcription real-time PCR to analyse the expression levels of the ECM33 gene family in the generated mutants

Reverse transcription real-time PCR analyses of ECM33 gene family (average of three independent experiments). The wild-type auxotroph strain $\Delta h t l$ expressed all genes that was tested and the generated mutant strains did not expressed the gene that was deleted. There was no compensatory up-regulation of SPS2/22 or PST1/ECM33 in the mutants. The housekeeping genes $A C T$ and $S L T$ were used for normalization.

\subsection{Generation of revertants and complemented strains}

All of the completed revertants (Apst1:CgPST1 and $\triangle e c m 33: C g E C M 33)$, incompleted revertants $\Delta p s t 1 / \Delta e c m 33: C g P S T 1$ and $\triangle p s t 1 / \triangle e c m 33: C g E C M 33$ and respective complemented

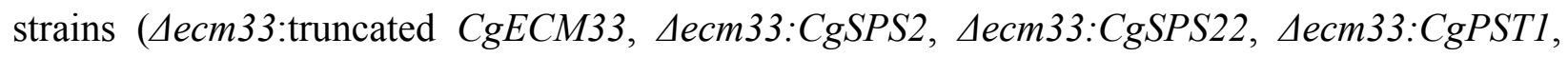
Aecm33:CaECM33 and $\triangle e c m 33: S c E C M 33)$ were generated by cloning the respective genes into expression plasmid pBM51 (Figure 6). Briefly, the inserted fragments were amplified with specific primers (Figure 15) and cloned to plasmid pBM51. All recombinant pBM51 candidates 
were verified with double restriction enzyme digestion as shown in Figure 16. The confirmed recombinant pBM51 plasmids were transformed into C. glabrata by electroporation. To verify the expression of revertant and complemented C. glabrata, a reverse transcription real-time PCR were performed for measurement of the level of target gene expression as shown in Figure 18.

All of the revertant strains expressed the inserted gene. The Apst1:CgPST1 and Apst1/Aecm33:CgPST1 could express PST1 in the same level as compared to the $\Delta h t l$ auxotrophic wild-type strain. $\triangle e c m 33: C g E C M 33$ and $\Delta p s t 1 / \Delta e c m 33: C g E C M 33$ expressed ECM33, but approximately $15 \%$ and $10 \%$ lower as compared to the $\Delta h t l$ auxotrophic strain. As demonstrated before, there was no PST1 and ECM33 expression in the respective mutants when the gene was deleted (Figure 17).

In complemented strains, Aecm33:truncated CgECM33, $\triangle e c m 33: C a E C M 33$, and $\triangle e c m 33: S c E C M 33$ could express ECM33 at levels around $70 \%, 90 \%$, and $90 \%$ of the $4 h t l$ auxotrophic strain, respectively. The strains $\triangle e c m 33: C g P S T 1, \triangle e c m 33: C g S P S 2$, and Aecm33:CgSPS22 contained two copies of PST1 and SPS2 and SPS22, respectively. However, there was only a low level of overexpression $(\sim 105 \%)$ of these genes in these strains, when compared to the $\Delta h t l$ auxotroph and the deletion mutant $\Delta e c m 33$ (Figure 18).

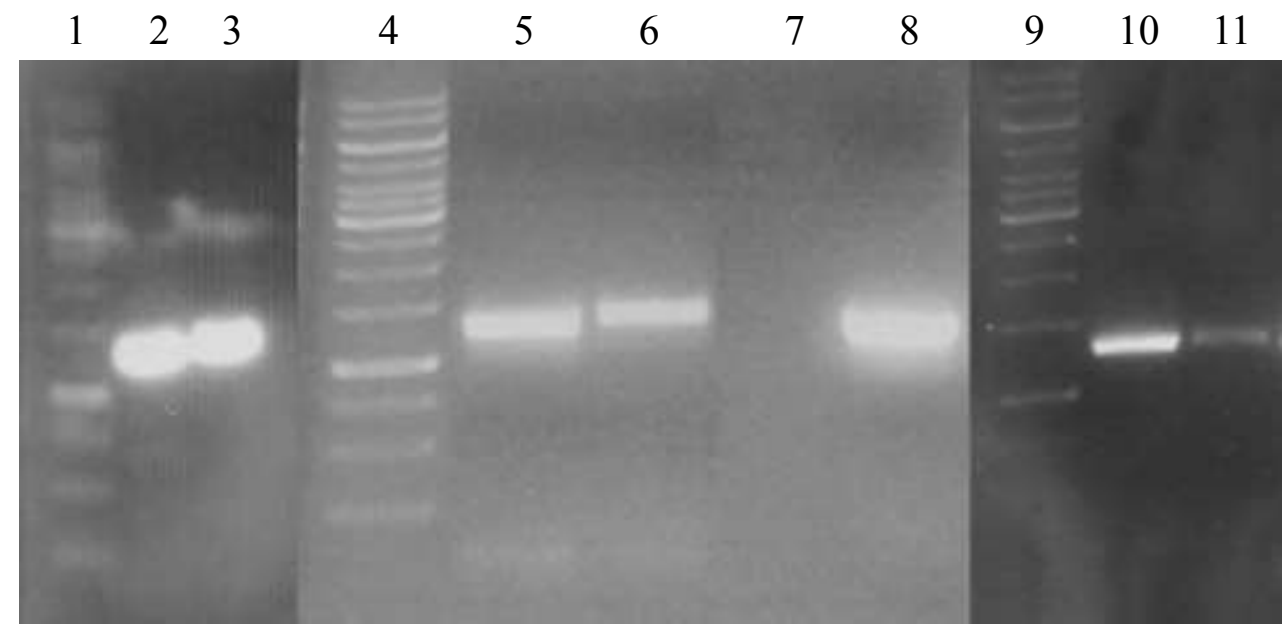

Figure 15. PCR products for recombinant pBM51 construction

PCR products of the interested genes were amplified from genomic DNA in case of C. glabrata and cDNA in case of $C$. albicans and $S$. cerevisiae with specific primers (Table 9). All lanes show a the expected specific amplification product. Non-specific PCR products were not observed. Lanes 1,4,9: DNA marker, lane 2: $C g E C M 33$, lane 3: $C g P S T 1$, lane 5: $C g S P S 22$, lane 6: $C g S P S 2$, lane 7: empty, lane 8: truncated $C g E C M 33$, lane 10: CaECM33 and lane 11: ScECM33 


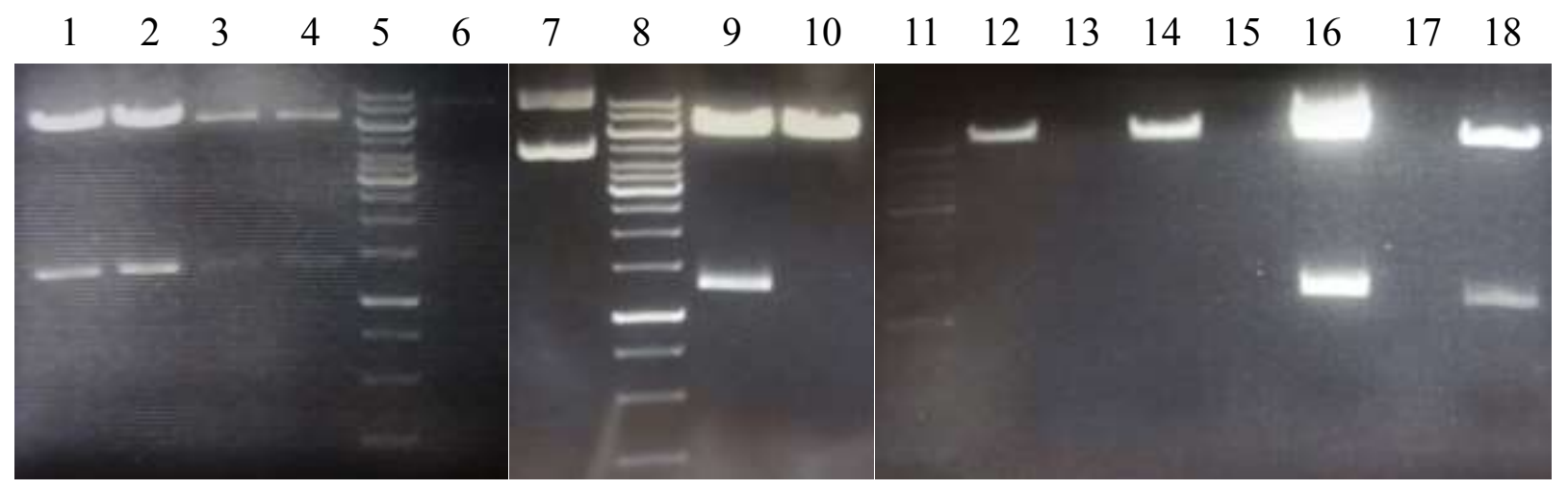

Figure 16. Enzyme digestion for verification of recombinant pBM51 plasmid

The recombinant pBM51 plasmids were extracted from the positive E. coli clones. Verification of these recombinant plasmids were performed by using restriction enzyme digestion. All recombinant plasmids containing the inserted genes show two bands on agarose gel. An upper band is pBM51 fragment and a lower band is the inserted gene. Lane 1: pBM51:CgECM33, lane 2: pBM51:CgPST1, lane 3: pBM51:CgSPS2, lane 4: pBM51:CgSPS22, lane 5,8,11: DNA marker, lane 6: BamHI digested pBM51:CgECM33, lane 7: two forms pBM51:truncated $C g E C M 33$ (upper is relaxed form and lower is supercoiled circular), lane 9: pBM51:truncated CgECM33, lane 10,12,14: pBM51, lane 13,15,17: empty, lane 16: pBM51:CaECM33, and lane 18: pBM51:SCECM33

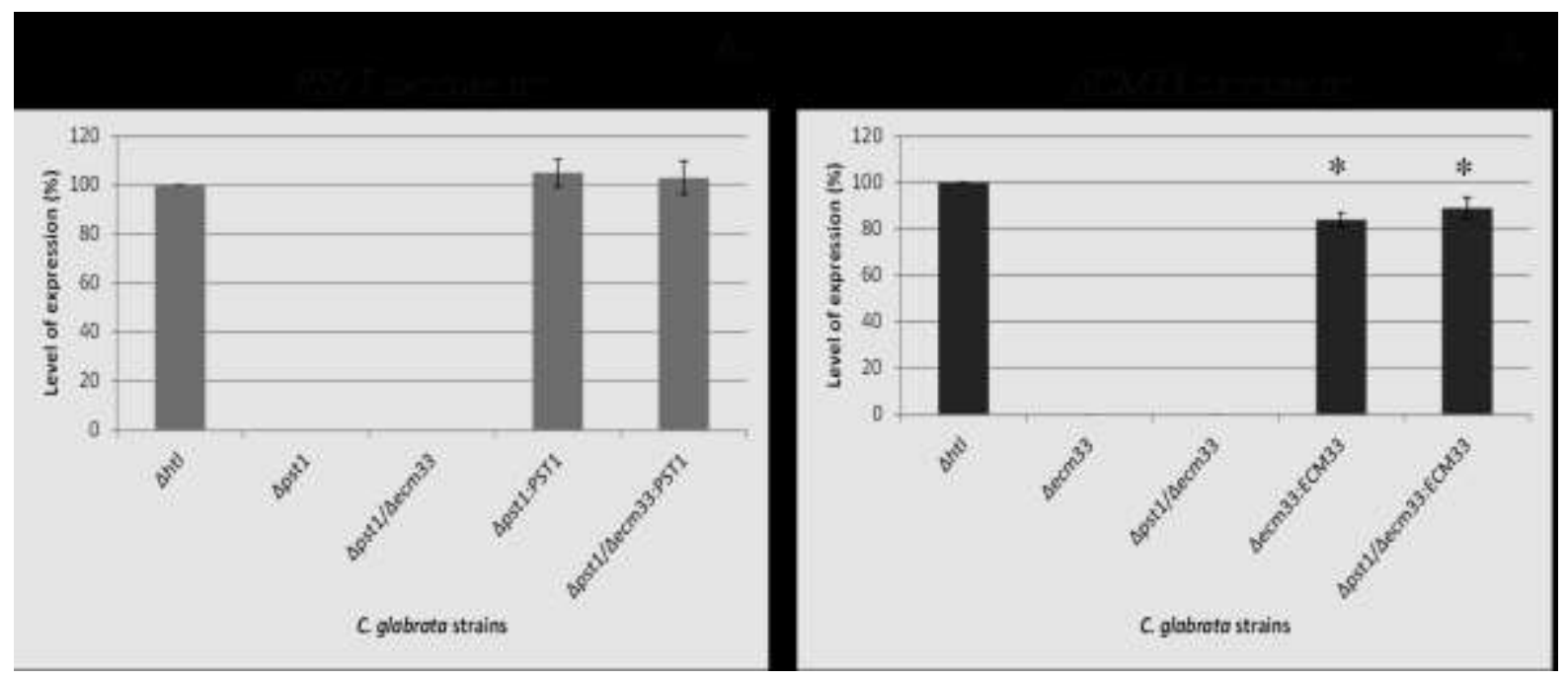

Figure 17. Reverse transcription real-time PCR for the measurement of PST1 and ECM33 gene expression in revertant strains

(A.): transcription levels of PST1; both PST1 revertants show PST1 gene expression as well as the $\Delta h t l$ auxotrophic strains (B.): transcription levels of ECM33; both ECM33 revertants show expression of the ECM33 genes but not in the same level as the $\Delta$ htl auxotrophic strains. *: statistically significant differences $(p<0.05)$. Error bars represent the mean value calculated from triplicate experiments $( \pm \mathrm{SD})$. 


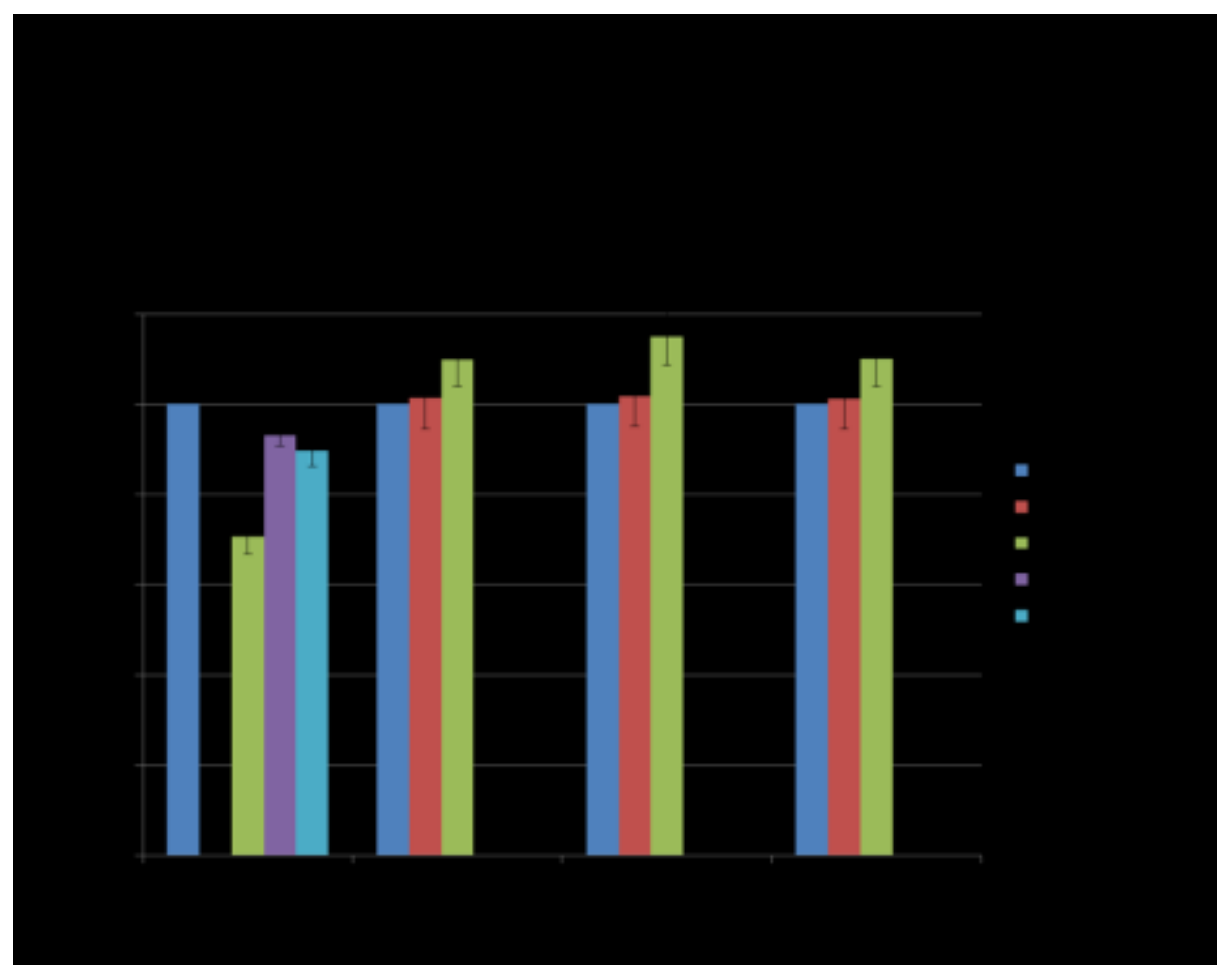

\section{Figure 18. Expression levels of the $E C M 33$ gene family members in complemented strains}

Relative quantification of gene expression of ECM33 gene family. The complemented strains of the ECM33 genes from C. glabrata, C. albicans and $S$. cerevisiae show expression of the introduced genes. Introduction of additional copies of PST1, SPS2, and SPS22 in $\triangle e c m 33$ did not lead to significant up-regulation when compared to $\triangle e c m 33$. The housekeeping genes $A C T$ and $S L T$ were used for normalization. *: statistically significant differences $(p<0.05)$. Error bars represent the mean value for triplicate experiments $( \pm \mathrm{SD})$.

\subsection{Growth rate determination}

To asses whether deletion of any of the genes leads to growth defects and if in such cases the phenotype was recovered in reconstitutet strains, growth rates in YPD and RPMI 1640 media were determined at both $30^{\circ} \mathrm{C}$ and $37^{\circ} \mathrm{C}$. Comparison of growth rates showed that all strains from C. albicans, S. cerevisiae, C. glabrata grew best in YPD at $37^{\circ} \mathrm{C}$ as shown in Figures 2022. 
The C. albicans mutant strain $\Delta e c m 33: \Delta e c m 33$ showed delayed growth as compared to the auxotrophic wild-type strain (Caf2) and its reconstituted control (Aecm33:Aecm33:ECM33:ECM33) under all conditions tested as shown in Figure 19.

In $S$. cerevisiae, the growth rate of the auxotrophic wild-type strain BY4741 was similar to the $4 p s t 1$ mutant. Moreover, the growth rate of the deletion mutant $\Delta e c m 33$ (BY4741 genetic background) was similar to the mutant $\triangle e c m 33$ (FBEH041 genetic background) but both $\triangle e c m 33$ strains grew slower when compared with the respective parental auxotrophic wild-type strains and the $\Delta p s t 1$ mutant. The $\Delta p s t 1 / \Delta e c m 33$ strain grew significantly slower than both, the wildtype and the single gene deletion mutants as shown in Figure 20.

In C. glabrata, the growth rate of wild-type strain CBS138/ATCC2001 was similar to the auxotrophic wild-type strain $\Delta h t l$ and $\Delta p s t 1$ mutant. The $\Delta e c m 33$ mutant grew slower than the wild-type and the $\Delta p s t 1$ mutant. The $\Delta p s t 1 / \Delta e c m 33$ mutant grew significantly slower than the wild-type, $\Delta p s t 1$, and $\Delta e c m 33$ as shown in Figure 21.

The growth rate of the revertants indicated that $\triangle e c m 33: \operatorname{CgECM} 33$, Apst1/Aecm33:CgPST1, and Apst1/Aecm33:CgECM33 grow slower than the auxotrophic wildtype strain $\Delta h t l$ and the $\Delta p s t 1$ mutamt, but faster than the $\Delta e c m 33$ and $\Delta p s t 1 / \Delta e c m 33$ as shown in Figure 21. Gene reversion can therefore restore the growth rate from the mutants close to approximate levels of the parental strain, as shown in Figure 21 and Figure $22 \mathrm{~A}$.

Complementations of CgECM33 with the ortholog from either $S$. cerevisiae or $C$. albicans rescued the growth defect in C. glabrata (Figure 23 B). However, addition of a second allele of either PST1, SPS2, or SPS22 under the ECM33 promotor does not rescue the ecm33 growth defect (Figure $23 \mathrm{~A}$ ). Neither does introduction of a truncated version of ECM33 without the GPI anchor attachment site (Figure $23 \mathrm{C}$ ). 


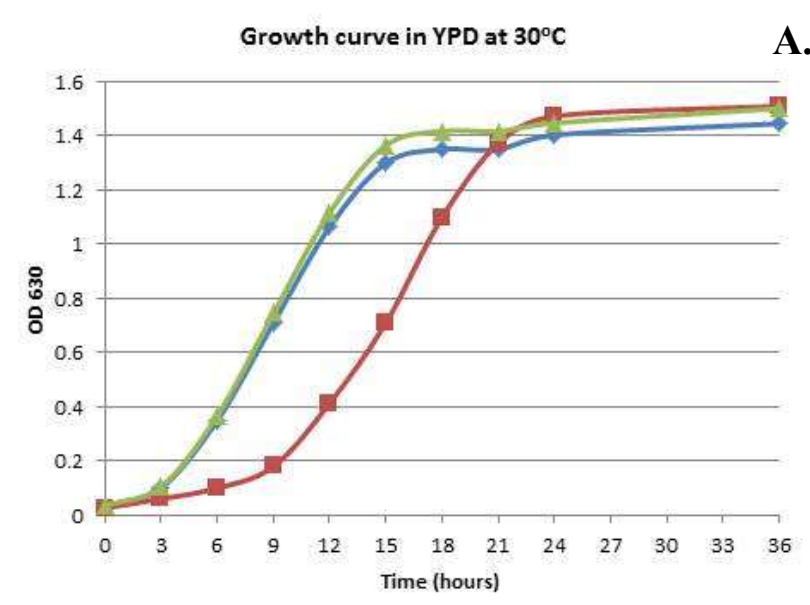

A.

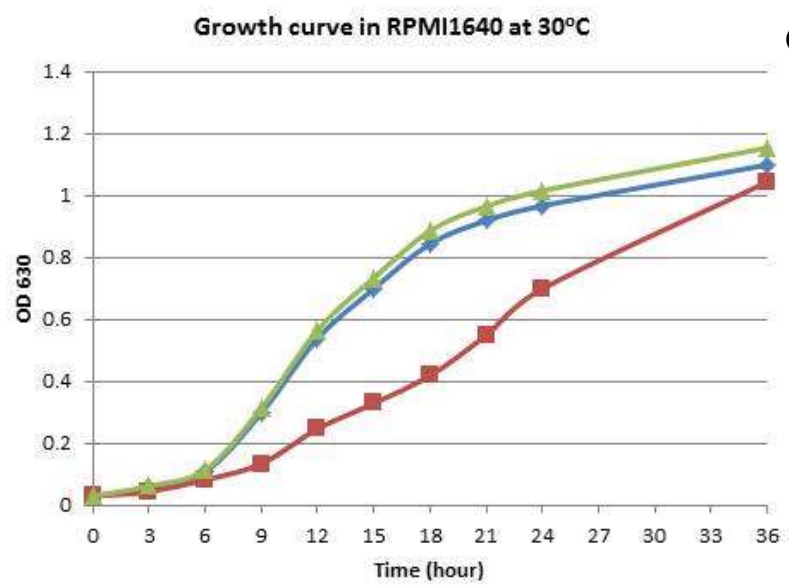

C.

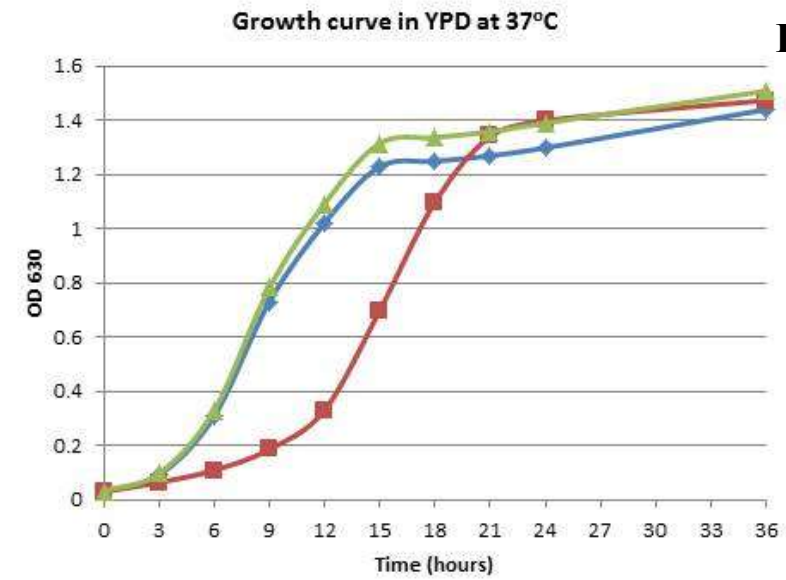

B.

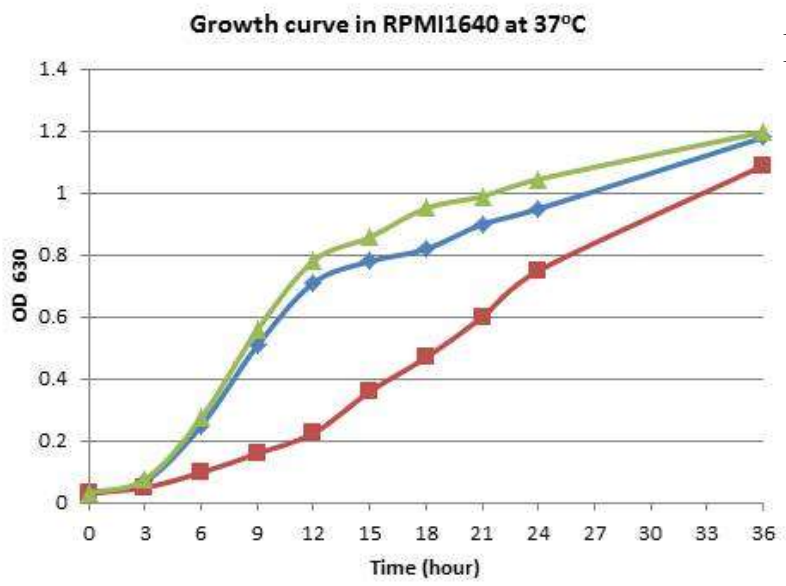

D.

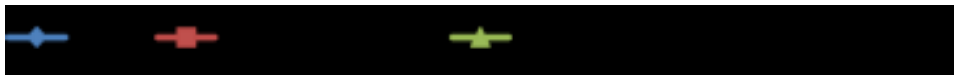

Figure 19. Growth assays of $C$. albicans mutants

(A.): YPD at $30^{\circ} \mathrm{C}$, (B.): $Y P D$ at $37^{\circ} \mathrm{C}$, (C.): RPMI 1640 at $30^{\circ} \mathrm{C}$, and (D.): RPMI 1640 at $37^{\circ} \mathrm{C}$. The cultures of $C$. albicans cells were grown in 96-well plates monitored for 36 hours and measured an optical density at $630 \mathrm{~nm}$. The $\Delta e c m 33: \Delta e c m 33$ mutant has a slower growth as compared with Caf2 auxotrophic wild-type. The reduced growth rate of the mutant was restored by complementing with CaECM33. 

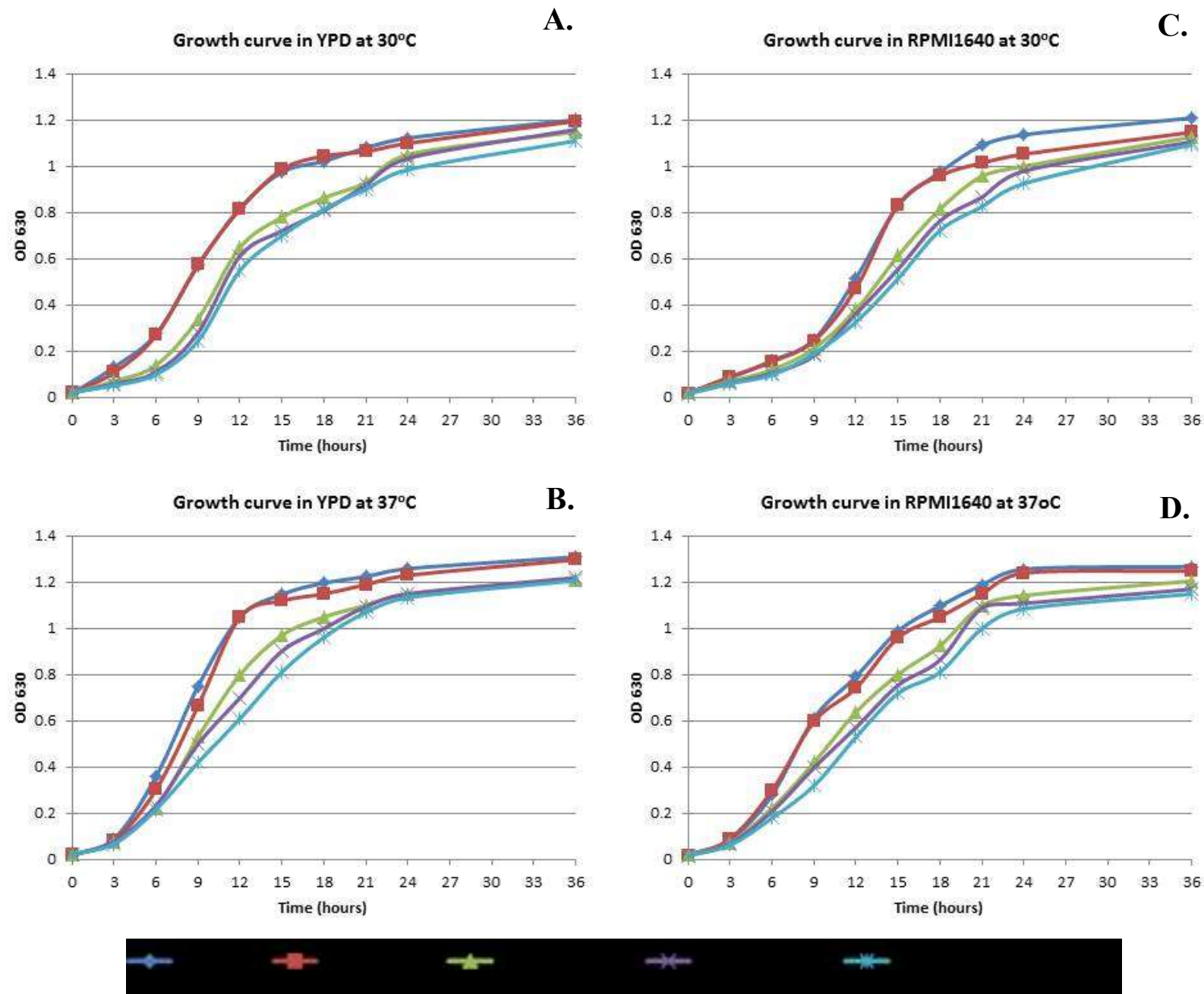

Figure 20. Growth assays of $S$. cerevisiae mutants

(A.): YPD at $30^{\circ} \mathrm{C}$, (B.): $Y P D$ at $37^{\circ} \mathrm{C}$, (C.): RPMI 1640 at $30^{\circ} \mathrm{C}$, and (D.): RPMI 1640 at $37^{\circ} \mathrm{C}$. The cultures of $S$. cerevisiae cells were grown in 96-well plates, monitored for 36 hours and measured an optical density at $630 \mathrm{~nm}$. The $\Delta e c m 33$ mutant has a slower growth as compared to the BY4741 auxotrophic wild-type and $\Delta p s t 1$ mutant. The $\Delta p s t 1 / \Delta e c m 33$ double mutant has the slowest growth as compared to all other strains. Control strains with the re-introduced genes were not available. Two separate genetic backgrounds were used to construct single and double deletion strains (Pardo, M., Monteoliva, L. et al., 2004). 

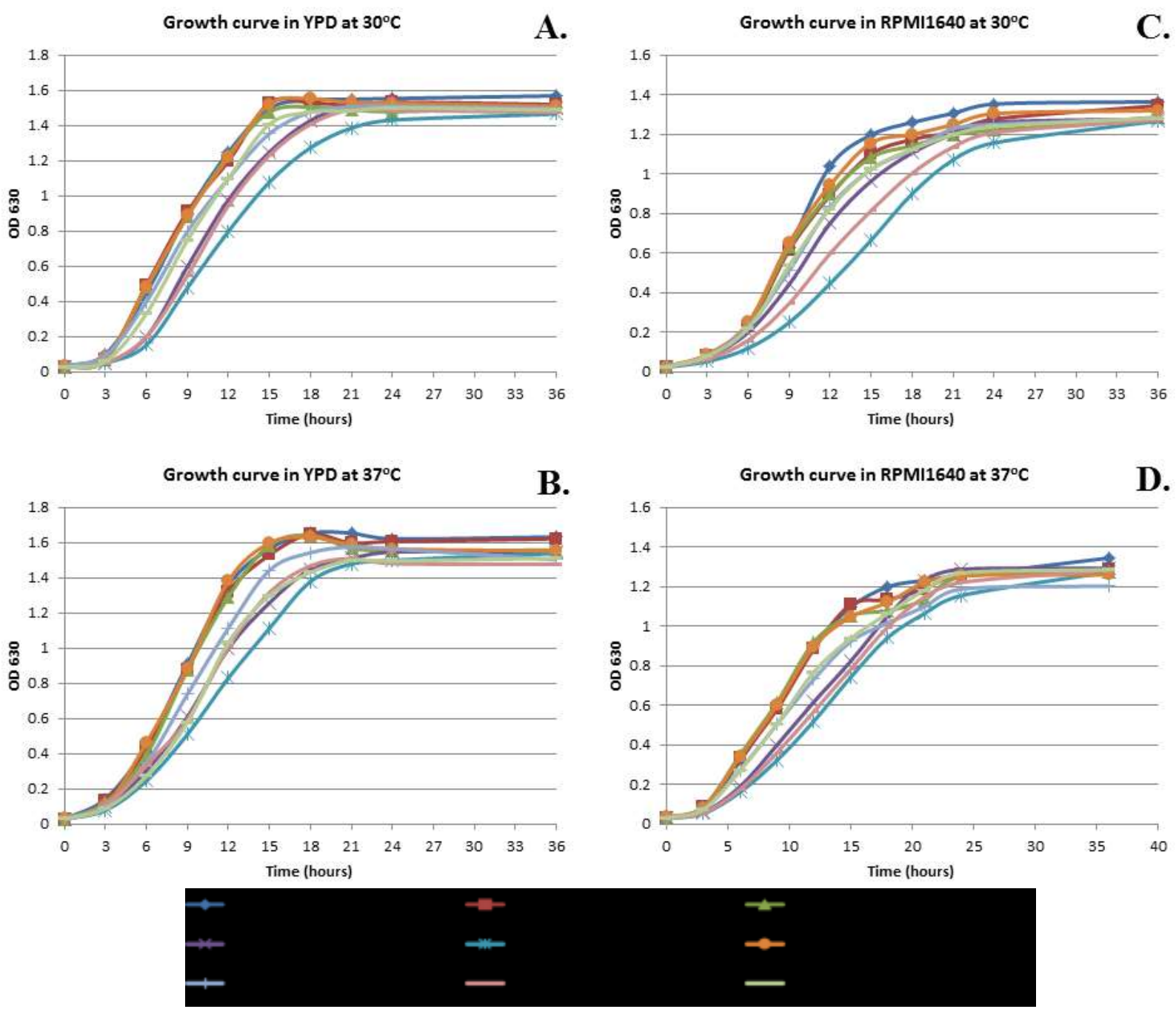

Figure 21. Growth assays of $C$. glabrata mutants

(A.): YPD at $30^{\circ} \mathrm{C}$, (B.): YPD at $37^{\circ} \mathrm{C}$, (C.): RPMI 1640 at $30^{\circ} \mathrm{C}$, and (D.): RPMI 1640 at $37^{\circ} \mathrm{C}$. The cultures of $C$. glabrata cells were grown in 96-well plates, monitored for 36 hours and measured an optical density at $630 \mathrm{~nm}$. The $\Delta e c m 33$ mutant has a slower growth as compared to the wild-type and $\Delta p s t 1$ mutant. The $\Delta p s t 1 / \Delta e c m 33$ mutant has the slowest growth as compared to all other strains. The reduced growth rate of the mutant was restored by complementing with CgECM33. 


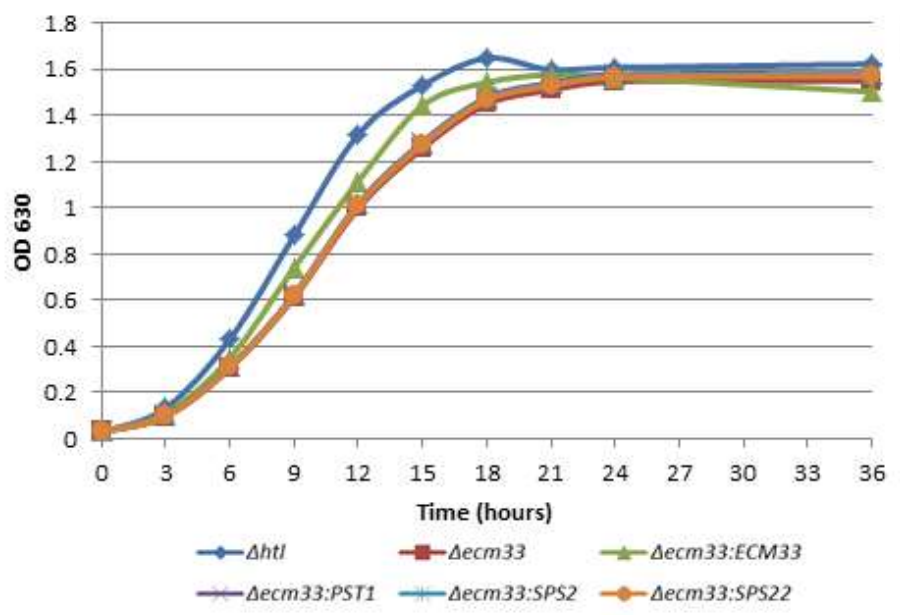

A.

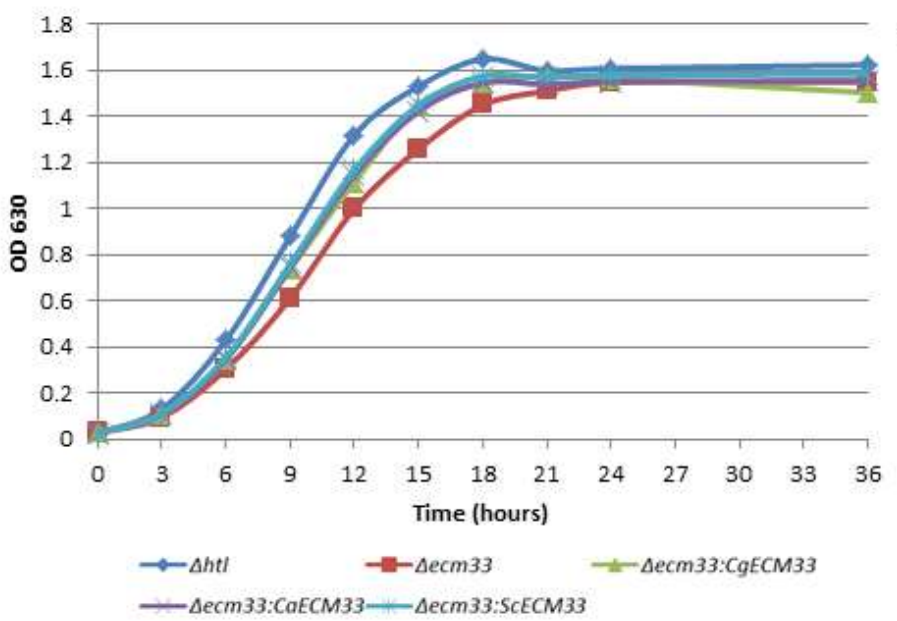

B.

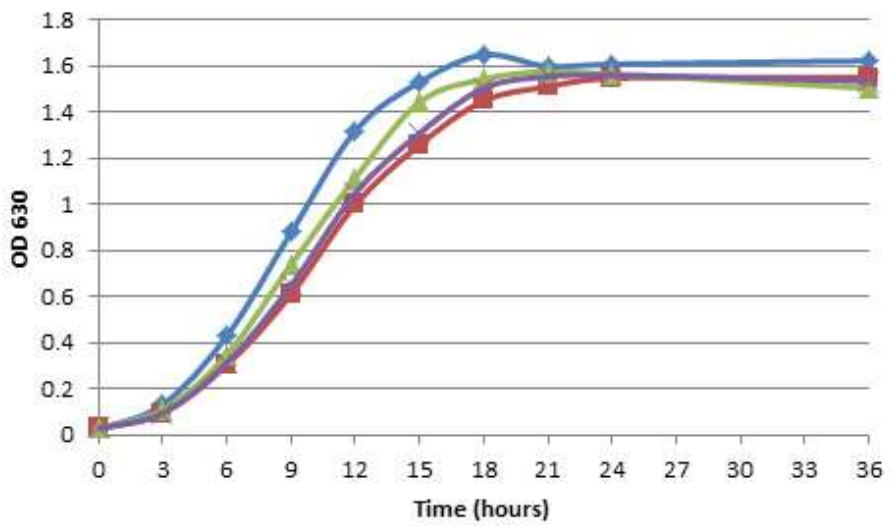

C.

$\rightarrow-\Delta t_{t} \quad \rightarrow-\Delta e c m 33$

$\longrightarrow-\triangle e c m 33: C g E C M 33 \quad \longrightarrow \Delta e c m 33:$ truncated CgECM33

Figure 22. Growth curve of complementation experiments $C$. glabrata in YPD at $37^{\circ} \mathrm{C}$

C. glabrata strains were determined in 96-well plates, monitored for 36 hours and measured an optical density at $630 \mathrm{~nm}$. Growth comparisons of (A.): strains complemented with C. glabrata ECM33, PST1, SPS2, and SPS22. (B.): complemented strains using C. albicans and S. cerevisiae ECM33. (C.): complemented strains using truncated $C g E C M 33$. 


\subsection{Phenotypic analysis}

\subsubsection{Measurement of cell wall strength}

To measure the effect of the different gene deletions on cell wall strength, growth assays on solid media supplemented with different concentrations of known cell-wall perturbing agents were carried out. As a starting point, previously published concentrations for the respective strains from C. albicans (Martinez-Lopez, R., Monteoliva, L. et al., 2004) and S. cerevisiae (Pardo, M., Monteoliva, L. et al., 2004) were chosen.

In $C$. albicans, the phenotypic differences between the $\Delta e c m 33$ mutant and the auxotrophic wild-type strain (Caf2) when exposed to $5 \mathrm{mM}$ Caffeine, $50 \mu \mathrm{g} / \mathrm{ml}$ Calcofluor white, and $200 \mu \mathrm{g} / \mathrm{ml}$ Congo red were seen (Figure $23 \mathrm{~B}$ ).

Similarly, for $S$. cerevisiae the $\Delta p s t 1$ mutant had no phenotypic difference compared to the the auxotrophic wild-type strain BY4741, but two independent $\Delta e c m 33$ mutants, showed a significantly decreased growth at concentrations of $10 \mathrm{mM}$ Caffeine, $10 \mu \mathrm{g} / \mathrm{ml}$ Calcofluor white, and $50 \mu \mathrm{g} / \mathrm{ml}$ Congo red. The $\Delta p s t 1 / \Delta e c m 33$ mutant also displayed a increased susceptibility in these assays (Figure $24 \mathrm{C}$ ).

However, there were no phenotypic differences among C. glabrata wild-type, auxotrophic wild-type, single mutants and double mutant when tested with $10 \mathrm{mM}$ Caffeine, 500 $\mu \mathrm{g} / \mathrm{ml} \mathrm{Calcofluor}$ white, and $500 \mu \mathrm{g} / \mathrm{ml}$ Congo red (Figure $23 \mathrm{~A}$ ). Of note, these concentrations were several fold higher in C. glabrata than the concentrations necessary to assay the phenotypes in C. albicans and $S$. cerevisiae.

Therefore, additional liquid culture (broth microdilution) assays were performed to confirm these data. The minimum inhibitory concentration (MIC)50- and MIC90-values were used as the indicators for a quantitative evaluation in sensitivity testing.

When the wild-type strains of C. glabrata, C. albicans and S. cerevisiae were compared in these assays, the CFW and Congo red MIC50- and MIC90-values were significantly higher in C. glabrata confirming that this organism is more resistant to cell perturbing agents. 

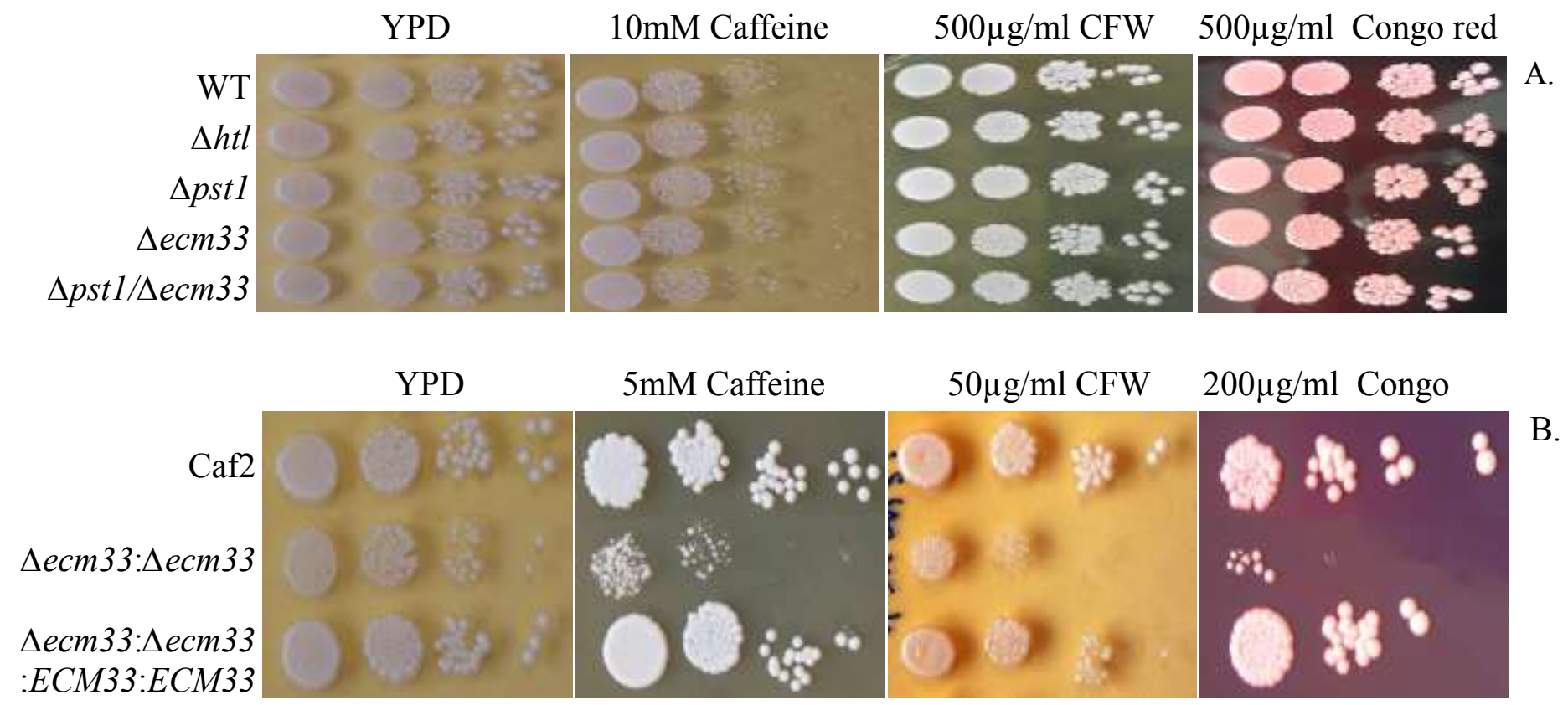

B.

:ECM33:ECM33

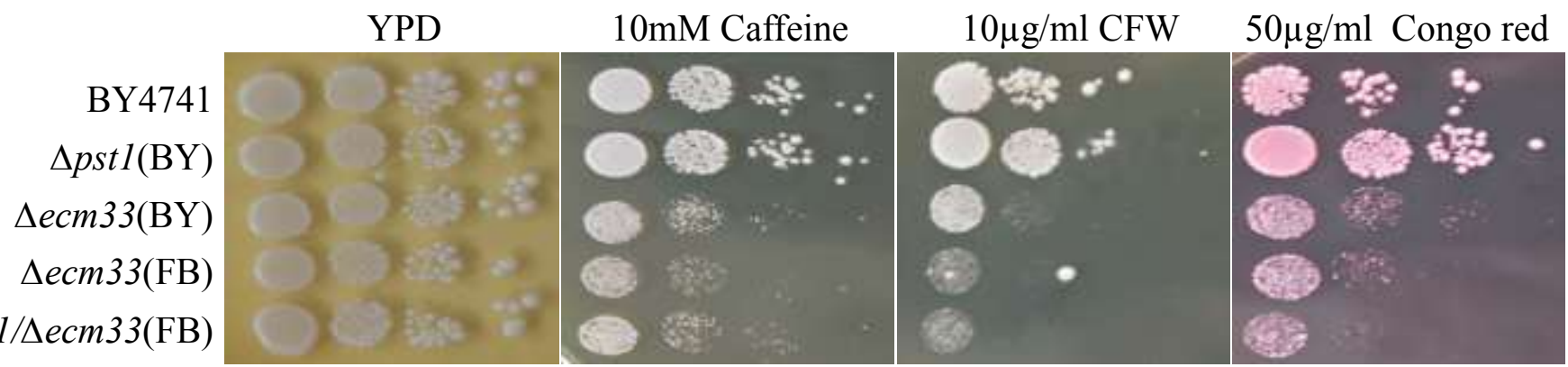

Figure 23. Sensitivity testing of $C$. glabrata, $C$. albicans and $S$. cerevisiae to cell perturbing agents by using agar drop dilution assay

(A): C. glabrata, (B): C. albicans, and (C): S. cerevisiae. The ECM33 gene deletion of $C$. albicans and $S$. cerevisiae resulted in the expected susceptibility to cell wall perturbing agents ((Martinez-Lopez, R., Monteoliva, L. et al., 2004; Pardo, M., Monteoliva, L. et al., 2004)); the $\Delta e c m 33$ mutant of $C$. albicans and the $\Delta e c m 33$ and $\Delta p s t / \Delta e c m 33$ mutants of $S$. cerevisiae are susceptible to Caffeine, CFW, and Congo red as compared to wild-type auxotrophic strain. However this effect was not observed in C. glabrata. 
Using liquid culture assays the C. glabrata $\Delta e c m 33$ mutant was moderately more sensitive to CFW and Congo red as compared to the wild-type strains. In the double mutant $\Delta p s t 1 / \Delta e c m 33$, these phenotypes were strongly aggravated (Table 14). The broth microdilution assays confirmed that the complemented $C$. glabrata mutants regained their cell wall rigidity. For example, the MIC50 for CFW of the 4 ecm 33 mutant is $250 \mu \mathrm{g} / \mathrm{ml}$, but increases to 500 $\mu \mathrm{g} / \mathrm{ml}$ in the revertant $\triangle e c m 33: C g E C M 33$ and reaches the level of the wild-type and auxotrophic strain (Table 14).

In contrast, when CgPST1, CgSPS2 or CgSPS22 was introduced into the $\triangle e c m 33$ mutant (4ecm33:CgPST1, $\triangle e c m 33: C g S P S 2$, and $\triangle e c m 33: C g S P S 22)$, the phenotype was not rescued, indicating that these homologs can not take over the function of ECM33 in C. glabrata (Table 14). Furthermore, a truncated version of $C g E C M 33$ without the GPI anchor site was not able to restore the phenotypic defects of the $\Delta e c m 33$ mutant in C. glabrata. In all phenotypic assays, the $\triangle e c m 33$ :truncated CgECM33 showed the same MIC50- and MIC90-values as compared to $\triangle e c m 33$ (Table 14). These phenotypic differences of the revertants (Aecm33:truncated CgECM33 vs $\triangle e c m 33: C g E C M 33)$ show that the GPI anchor site of the Ecm33 protein is necessary for native Ecm33 function.

Interestingly, the C. albicans and S. cerevisiae orthologs of CgECM33 (CaECM33 and ScECM33) were able to restore the phenotypes of $C g \Delta e c m 33$ and the revertants Aecm33:CaECM33 and Aecm33:ScECM33 display similar MIC50- and MIC90-values as the C. glabrata wild-type strain.

In $C$. albicans, the mutant strain $\Delta e c m 33: \Delta e c m 33$ was more sensitive to all cell perturbing agents when compared to the auxotrophic wild-type strain (except Lufenuron) and the revertant strains showed the same MIC50- and MIC90-values as the wild-type auxotrophic strain for all tested perturbants (Table 14).

In S. cerevisiae neither Brefeldin A, Nikkomycin Z nor Lufenuron did not inhibit the growth of the yeast cells in the tested concentration range. The two strains of $\Delta e c m 33$ mutants were more sensitive to CFW, Congo red, and Hygromycin B. With all cell perturbing agents, the Apst1 mutant showed similar phenotypes as compared to the wild-type auxotroph (BY4741). However, the $4 p s t 1 / \Delta e c m 33$ mutant was found to be four-times more sensitive when compared to the wild-type auxotrophic strain or the $\Delta$ pst 1 mutant (Table 14). 
Table 14. Summary of results obtained by broth microdilution assay

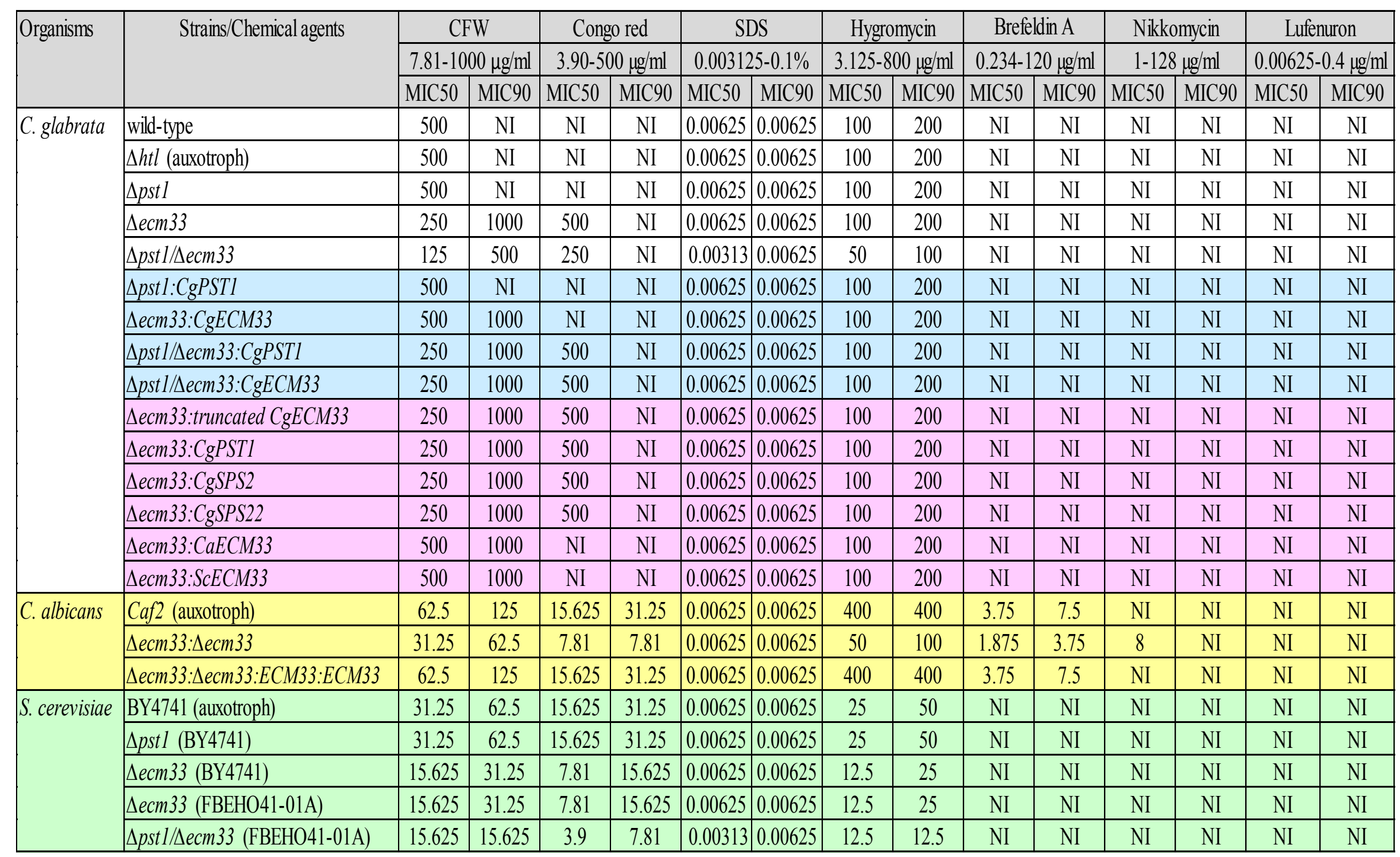

MIC50 and MIC90: the minimum inhibitory concentration 50 and 90\%, NI: no-inhibition. 


\subsubsection{Determination of cell surface charge}

Alcian blue is a positively charged dye that strongly binds to negative charges on fungal cell walls. Binding of Alcian blue (i.e. the reduction of absorbance in the reaction buffer) was used to quantify the charge state of cell walls. These assays demonstrated that specifically the deletion of ECM33 led to increased Alcian blue binding in the cell wall of C. glabrata, C. albicans and S. cerevisiae. This effect was observed in ECM33 deletions (Aecm33 and $\Delta p s t 1 / \Delta e c m 33$ mutants). However, it was not observed in the $\Delta p s t 1$ mutants (Figure 24). In the complemented strains of $C$. glabrata were not significantly different as compared to wild-type.
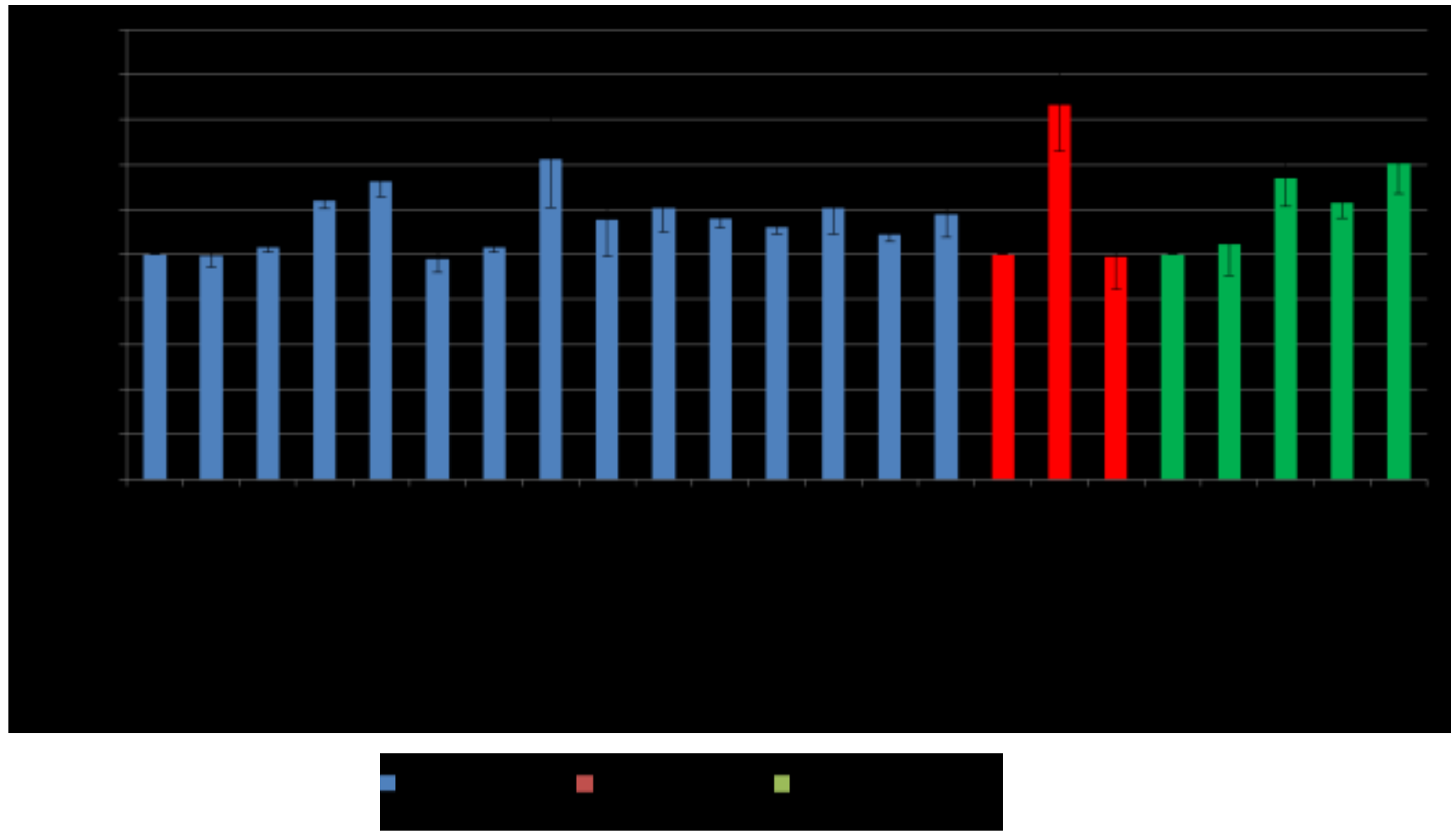

Figure 24. Alcian blue binding of $C$. glabrata, $C$. albicans and $S$. cerevisiae

Binding of Alcian blue normalized to the respective wild-type strains ( $=100 \%)$. *: statistic test by student's t-test (significance threshold $p<0.05$ ). Error bars represent the mean value calculated from triplicate experiments $( \pm \mathrm{SD})$. 


\subsubsection{Determination of cell wall composition}

Fluorescence-activated cell sorting (FACS) assays were used to quantify the binding of different substances to the cell wall components glucan and chitin. Alexa-fluor 647-labelled wheat germ agglutinin (WGA) was used to stain chitin and Congo red to stain glucan in the fungal cell wall. Quantification by FACS analysis demonstrated that ECM33 deleetion (Aecm33 or $\Delta p s t 1 / \Delta e c m 33)$, but not $\Delta p s t 1$ mutant led to an increased WGA staining in C. glabrata, $C$. albicans and S. cerevisiae (Figure $25 \mathrm{~A}$ ).

Overexpression of CgPST1, CgSPS2 or CgSPS33 in the $\triangle e c m 33$ mutant strain (4ecm33:CgPST1, $\triangle e c m 33: C g S P S 2$, and $\triangle e c m 33: C g S P S 33)$ did not compensate the increased WGA binding. However, expression of ECM33 from C. albicans and S. cerevisiae rescued this phenotype in the ECM33 deletion of C. glabrata (Figure $25 \mathrm{~A}$ ).

Consistantly, in all three species examined, no significant differences of the mutants in Congo red binding was observed, implying that the lack of ECM33 does not affect the total glucan levels of the cells (Figure $25 \mathrm{~B}$ ).

\subsubsection{Measurement of adherence properties}

To examine the adherence ability of the yeast cells, adherence assays towards polystyrene and silicone were carried out. In addition, the cell surface hydrophobicity was determined by measuring relocalization of cells from an aqueous to a hydrophobic phase (octane test). These hydrophobicity assays showed that the C. glabrata Aecm33 mutant was significantly less hydrophobic as compared to the wild-type and auxotrophic strain. In addition, the $C$. glabrata $4 p s t 1 / \Delta e c m 33$ mutant was found to be significantly less hydrophobic as compared to the single $\triangle e c m 33$ mutant. In contrast, the loss of PST1 alone did not affect the hydrophobicity. There were no significant differences of cell surface hydrophobicity among the gene deletion strains in $C$. albicans and $S$. cerevisiae when compared to wild-type auxotrophic strains (Figure 26 A). 


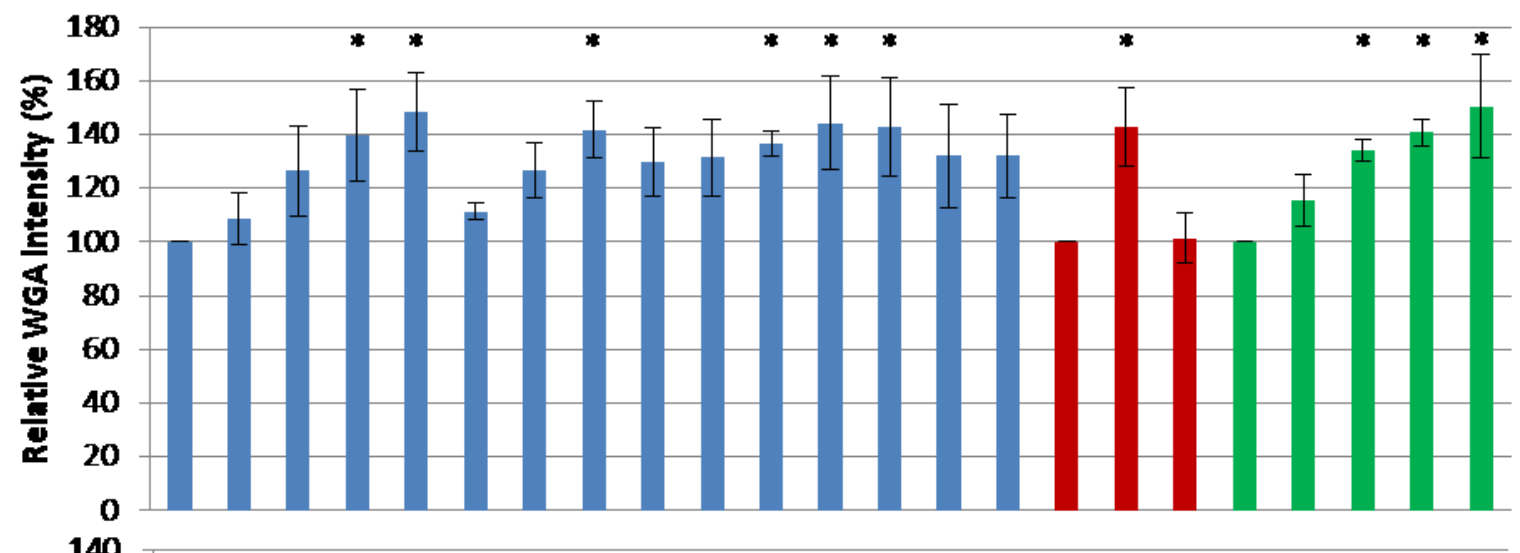

B.

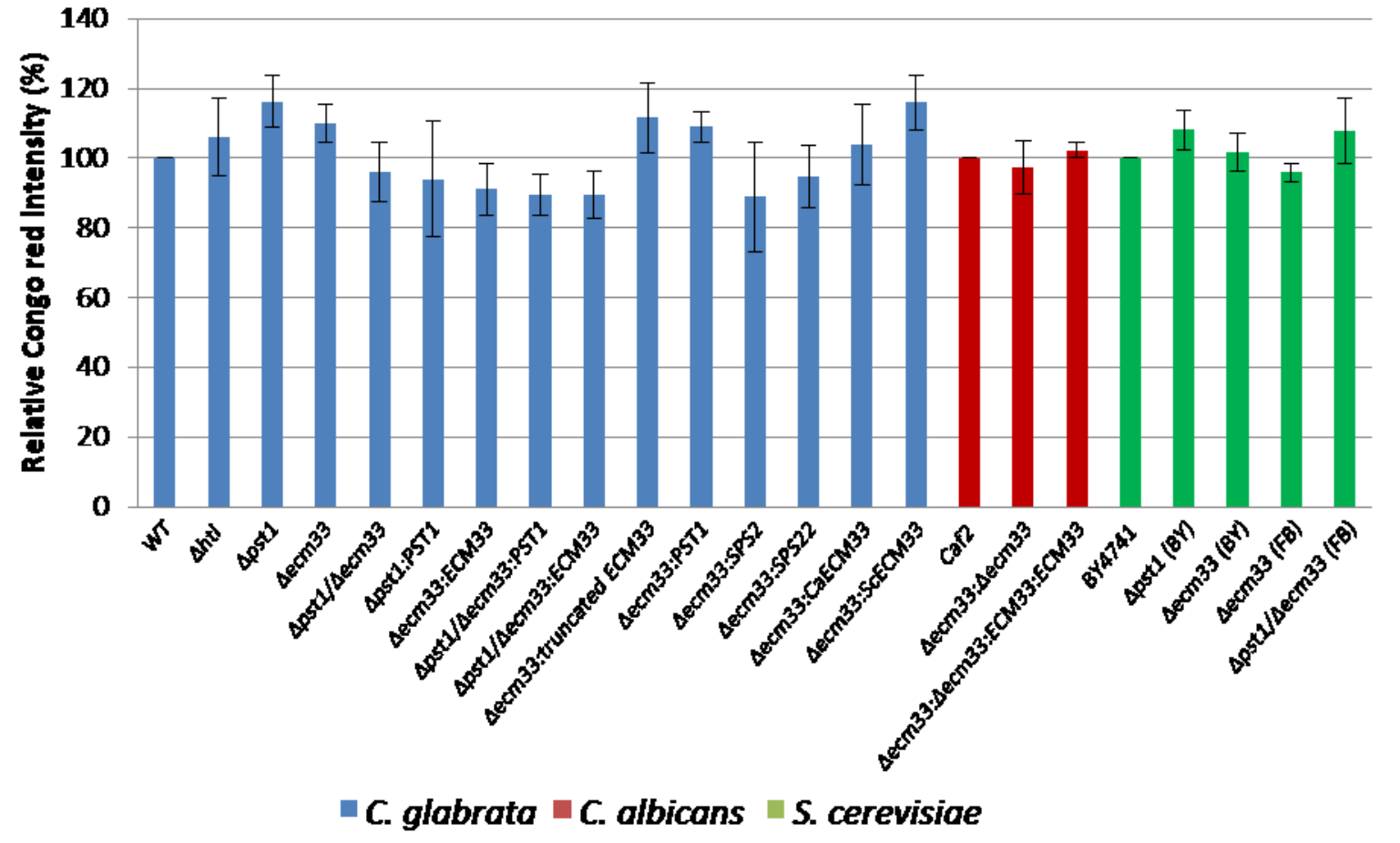

Figure 25. FACS analysis of WGA and Congo red binding in $C$. glabrata, $C$. albicans and $S$. cerevisiae

Realtive staining of chitin (A: Alexa Fluor ${ }^{\circledR} 647$ conjugated wheat germ agglutinin) and glucan (B: Congo Red) normalized to the respective wildtype strains $(=100 \%)$, as measured by FACS. *: statistic test by student's t-test (significance threshold $p<0.05$ ). Error bars represent the mean value calculated from triplicate experiments $( \pm \mathrm{SD})$. 
In polystyrene adherence assays, the C. glabrata $\Delta e c m 33$ and $\Delta p s t 1 / \Delta e c m 33$ mutants were significantly less adherent as compared to the wild-type and auxotrophic strains. There were no significant differences between strains of $C$. albicans and $S$. cerevisiae, respectively (Figure 26 B).

In these assays, the results of the hydrophobicity tests show a high correlation to the results of polystyrene tests. For example, the strains showing high hydrophobicity properties also showed, high polystyrene adherence capacities.

The silicone adherence capacity of $C$. glabrata was significantly reduced in the $\Delta e c m 33$ mutant when compared to the wild-type and auxotrophic strains. Additionally, the double mutant $\Delta p s t 1 / \Delta e c m 33$ showed a further significant reduction of about $60 \%$ in adherence towards silicone when to the $\triangle e c m 33$ mutant, which was rescued in the respective reconstituted controls.

Strains of C. glabrata heterologously complemented with CgPST1, CgSPS2, CgSPS22, ScECM33 and CaECM33 showed no statistically significant differences when compared to the parental $\triangle$ ecm 33 strain.

In C. albicans, the $\Delta e c m 33$ mutant showed a similar reduced silicone adherence capacity. In S. cerevisiae this effect was not observed, since the S. cerevisiae wild types did not adhere to silicone at all, as shown in Figure $26 \mathrm{C}$. 


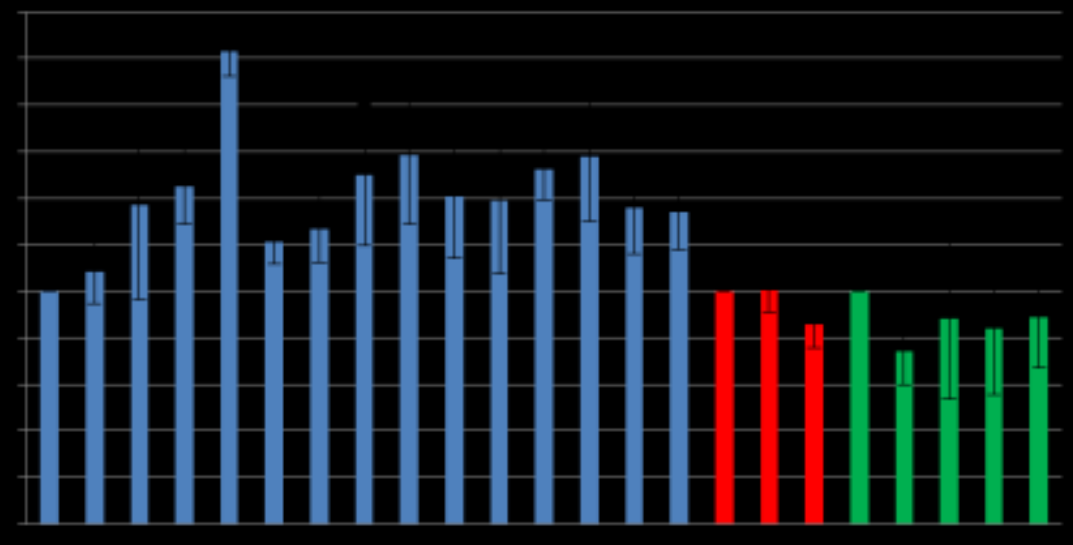

A.
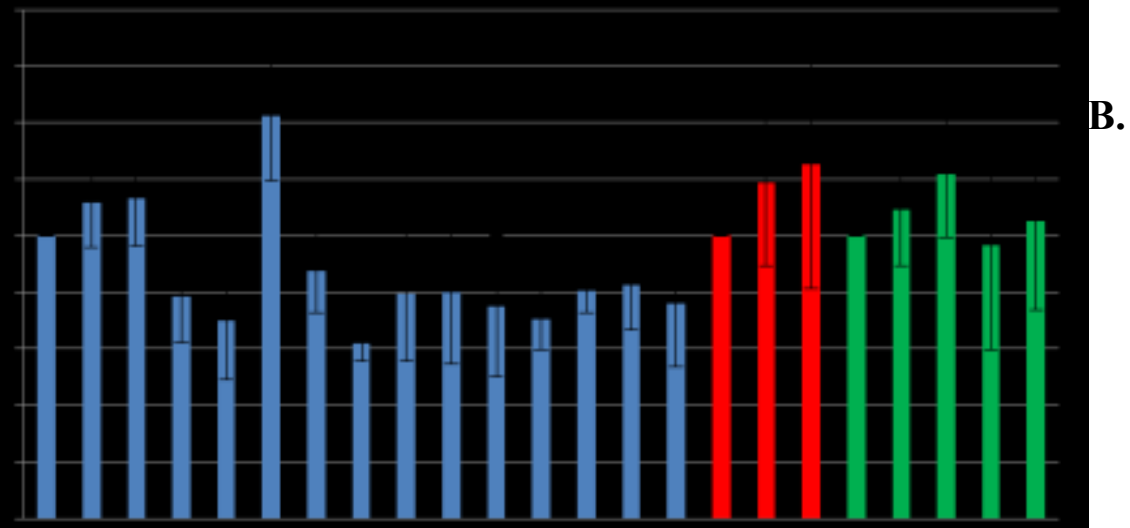

B.
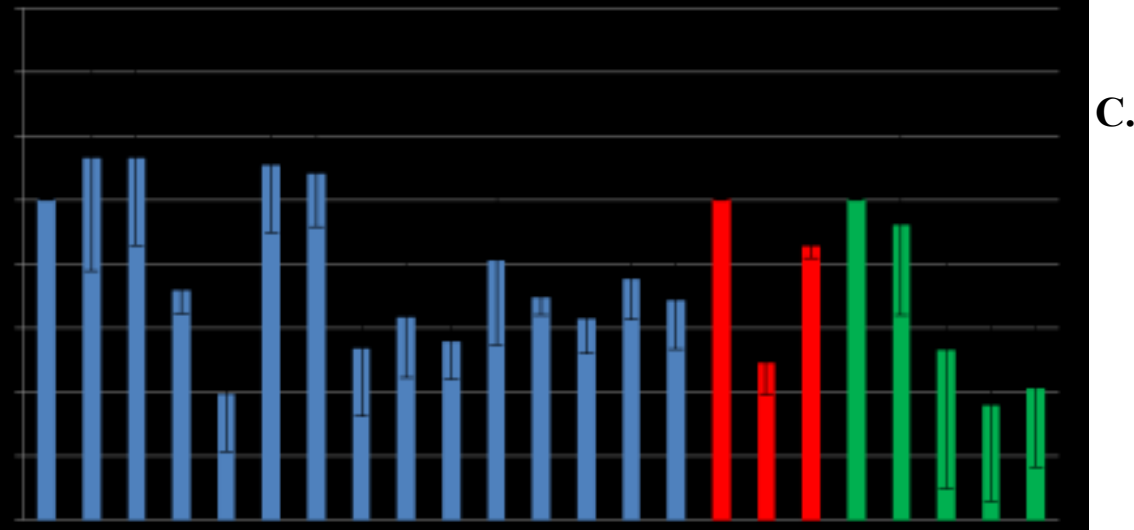

C.

Figure 26. Adherence properties of $C$. glabrata, $C$. albicans and $S$. cerevisiae.

Realtive hydrophilicity and adherence proterties normalized to the respective auxotrophic wildtype strains (=100\%), (A): hydrophilicity, (B): polystyrene adherence capacity, and (C): silicone adherence capacity. *: statistic test by student's t-test as (significance threshold $p<0.05$ when compared to auxotrophic wild-type strain). $* *$ : significance threshold $p<0.05$ when compared with $\Delta e c m 33$. Error bars represent the mean value calculated from triplicate experiments $( \pm \mathrm{SD})$. 


\subsection{C. glabrata Ecm33 and Pst1 protein expression in P. pastoris}

\subsubsection{Recombinant pPICZa vector construction}

The pPICZ $\alpha$ vecor was used for expression of recombinant Ecm33 and Pst1 proteins (EasySelect $^{\mathrm{TM}}$ Pichia Expression Kit, Invitrogen). The partial CgECM33 and CgPST1 genes without respective signal peptides and $\omega$-sites were amplified and introduced into the pPICZ $\alpha$ plasmid. The PCR product size of $C g E C M 33$ and $C g P S T 1$ fragments were 1,150 and 1,183 bp, respectively (Figure $27 \mathrm{~A}$ ).

The recombinant $\mathrm{pPICZ} \alpha$ candidates were verified with double restriction enzyme digestion and plasmid DNA sequencing. The recombinant pPICZ $\alpha$ :CgECM33 candidate was digested with EcoRI and KpnI and the recombinant pPICZ $\alpha: C g P S T 1$ candidate was confirmed by EcoRI and XbaI. The CgECM33 and CgPST1 digested fragments have a size of 1,144 and $1,177 \mathrm{bp}$, respectively (Figure $27 \mathrm{~B}$ ). Confirmed vector plasmids were transformed into the $P$. pastoris strains X33 and GS115.

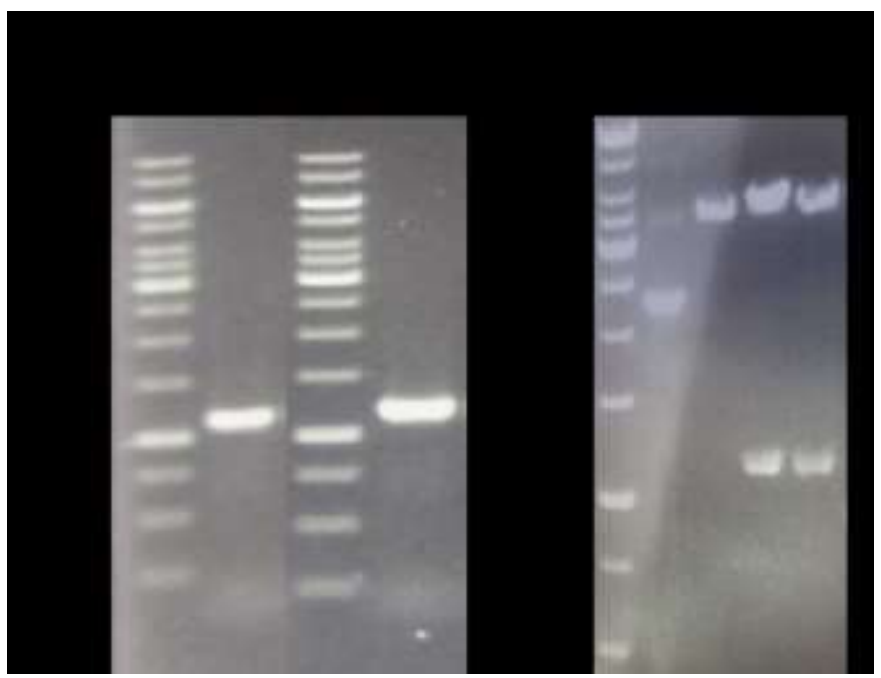

Figure 27. Amplification of $C g E C M 33$ and $C g P S T 1$ genes and verification of both recombinant pPICZ $\alpha$ plasmids

A: PCR products of $C g E C M 33$ and $C g P S T 1$ genes. Lane 1,4: DNA marker, lane 2: 1,150 bp of $C g E C M 33$, and lane 4: 1,183 bp of CgPST1.

$\mathrm{B}$ : verification of the recombinant pPICZ $\alpha: C g E C M 33$ and pPICZ $\alpha: C g P S T 1$ plasmids by using double restriction enzyme digestion. Lane 1: DNA marker, lane 2: natural pPICZ $\alpha$ (upper band: relaxed form and lower band: supercoiled), lane 3: pPICZ $\alpha$ digested with $E c o R \mathrm{I} / K p n \mathrm{I}$, lane 4: pPICZ $\alpha: C g E C M 33$ digested with EcoRI/KpnI, and lane 5: pPICZ $\alpha: C g P S T 1$ digested with EcoRI/XbaI 


\subsection{2 $C g$ Ecm 33 and $C g$ Pst1 proteins expression in $P$. pastoris}

Resulting from the procedure outlined above, four strains were generated to express recombinant gylcosylated $C g E c m 33$ and $C g$ Pst1 proteins in P. pastoris (GS115:CgECM33, GS115:CgPST1, X33:CgECM33, and X33:CgPST1). All strains were tested for recombinant protein expression and subsequently the conditions and time points of protein expression were optimized. The results of SDS-PAGE analyses showed that the P. pastoris supernatants from day 3 had the highest concentration of total proteins and that P. pastoris GS115 expresses both $C g$ Ecm 33 and $C g$ Pst1 proteins to a higher extend as compared to the X33 strains (Figure 28). Based on these results, the GS115:CgECM33 and GS115:CgPST1 strains were used to express the proteins for further studies.

In SDS-PAGE analyses, both proteins bands were visible as high molecular weight smears between 130 to $55 \mathrm{kDa}$ (compared to the calculated molecular weight deduced from the amino acid sequence of the recombinant $C g E c m 33$ and $C g P s t 1$ proteins as 41 and $42 \mathrm{kDa}$, respectively). To analyse whether the observed higher molecular weight of the recombinant proteins was caused by extensive glycosylation, deglycosylation experiments were performed.

Treatment of the recombinant proteins with N-glycosidase (Endo- $\alpha-\mathrm{N}-$ Acetylgalactosaminidase) resulted in a reduced molecular weight from around 100 to $72 \mathrm{kDa}$ to approximately, 55 and $60 \mathrm{kDa}$, respectively.and a more coherent band in SDS-PAGE gels as shown in Figure 29. However, no reduction in the molecular weight of both proteins was observed when O-glycosidase (PNGase F) was used (Figure 29).

Similarly, double digestion was little different from treatment with N-glycosidase (Figure 29). The results indicated that the recombinant Ecm 33 and Pst1 proteins which were produced in $P$. pastoris are heavily $\mathrm{N}$-glycosylated as digestion with $\mathrm{N}$-glycosidase resulted in a significant reduction of the molecular weight.

Recombinant Ecm33 and Pst1 proteins were used subsequently for determination of antibody specificity and cell-wall binding experiments. 


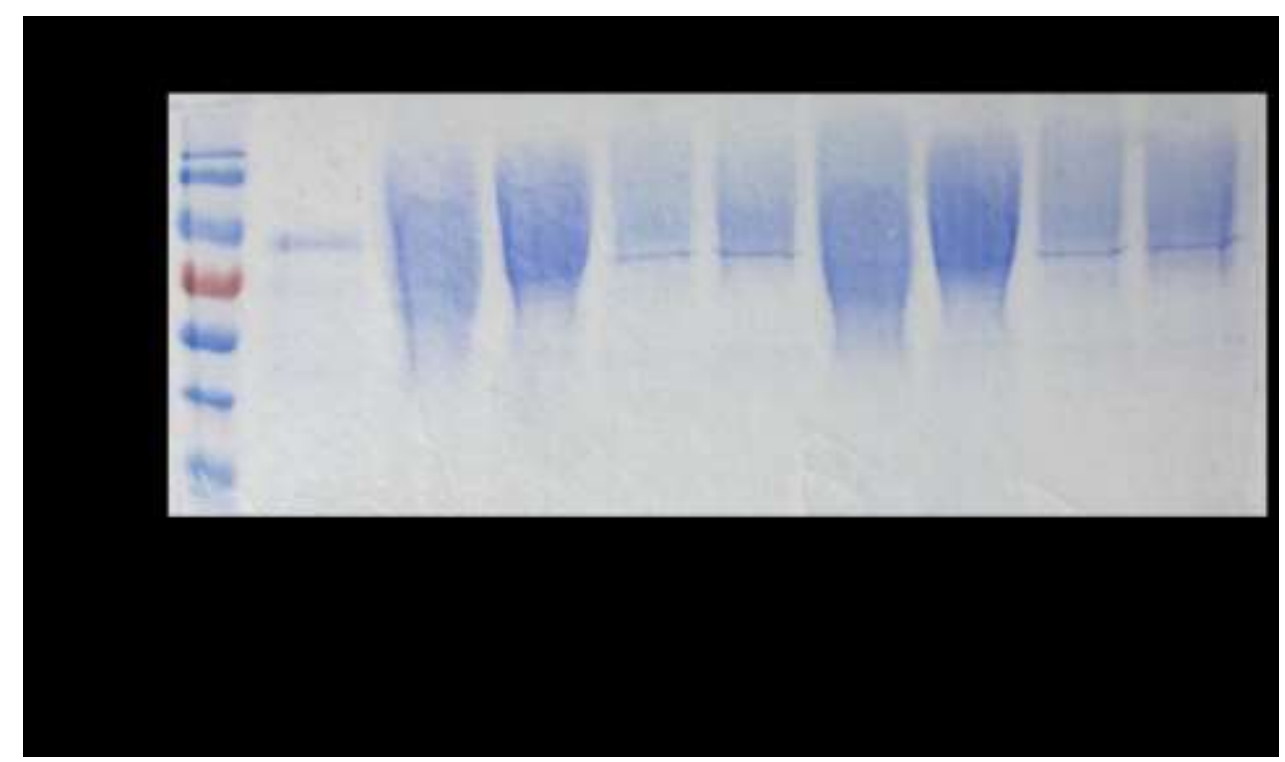

Figure 28. $C g$ Ecm33 and $C g P s t 1$ protein expression in P. pastoris

All recombinant $P$. pastoris strains were grown in $\mathrm{BMMH}$ media and incubated at $30^{\circ} \mathrm{C}$ with aeration for 2-3 days. Kex2 expression was used as a positive control of the protein expression (Lesage, G., Tremblay, M. et al., 2001). The GS115:CgECM33 and GS115:CgPST1 strains were used to express the proteins for further studies because of the highest protein production. Three days incubation is the best condition time for expression of $C g$ Ecm 33 and $C g$ Pst1 proteins.

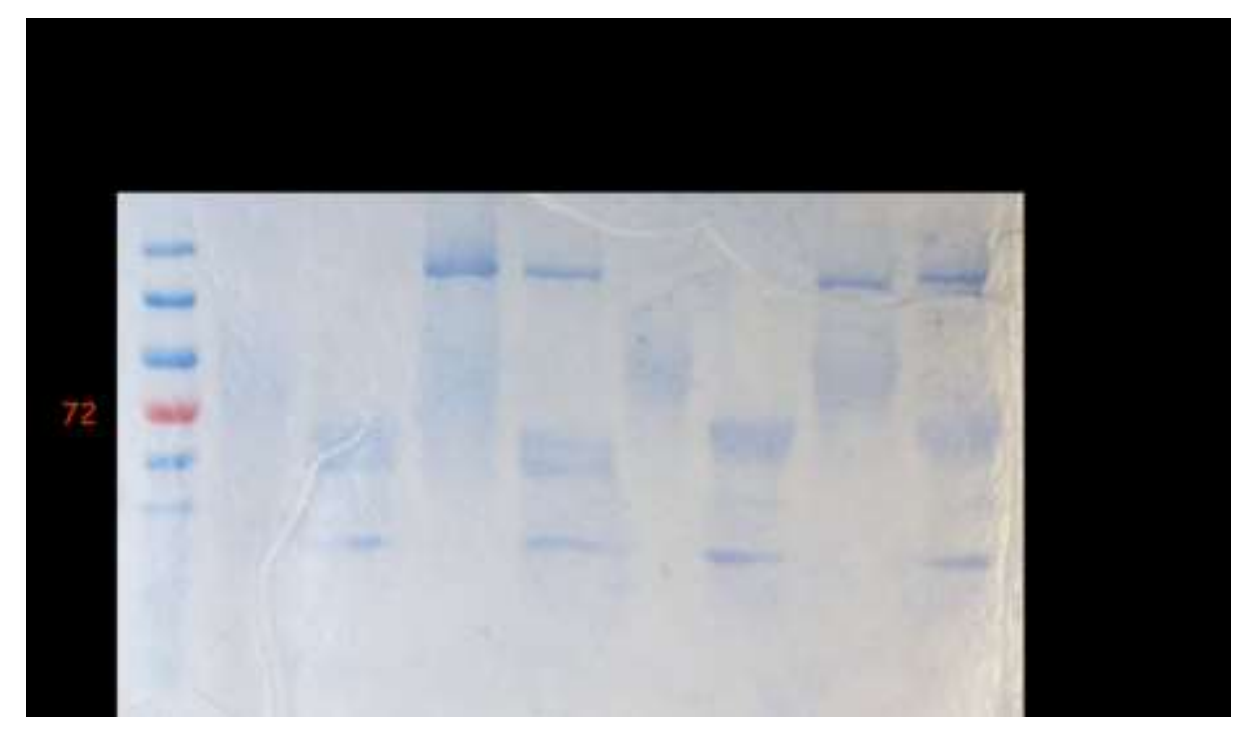

Figure 29. $C g E \mathrm{E} \mathrm{cm} 33$ and $C g$ Pst1 protein deglycosylation

$C g E \mathrm{Em} 33$ and $C g$ Pst1 recombinant proteins from P. pastoris were deglycosylated by using Nglycosidase, O-glycosidase, or by double digestion with $\mathrm{N}$-glycosidae and O-glycosidase. U: untreated, N: treatment with $\mathrm{N}$-glycosidase (molecular weight of $36 \mathrm{kDa}$ ), O: treatment with Oglycosidase (molecular weight of $147 \mathrm{kDa}$ ), and N/O: double treatment with $\mathrm{N}$-glycosidae and O-glycosidase. $C g E c m 33$ and $C g P s t 1$ from P. pastoris are N-glycosylated but do not appear to be O-glycosylated. 


\subsection{Anti-Ecm33 antibody production}

\subsubsection{Generation of plasmids for the expression of recombinant $C g E c m 33$ protein in $E$. coli}

Ecm33 protein expression in E. coli was performed using the QIAexpressionist ${ }^{\mathrm{TM}}$ system as described by the manufacturerer. The primers F-LM-pQE and R-LM-pQE were used to amplify a 1,150 bp of ECM33 fragment (Figure $30 \mathrm{~A}$ ) and cloned to the BamHI/PstI linearlized pQE30 plasmid. The generated pQE30:CgECM33 plasmid was confirmed using double restriction enzyme digestion and plasmid DNA sequencing. The recombinant pQE30:CgECM33 candidate was digested with BamHI and Pst1. The CgECM33 digested fragment has a size of 1,144 bp as shown in Figure $30 \mathrm{~B}$. After verification, the pQE30:CgECM33 plasmid was transformed to the competent E. coli strain BL-21.

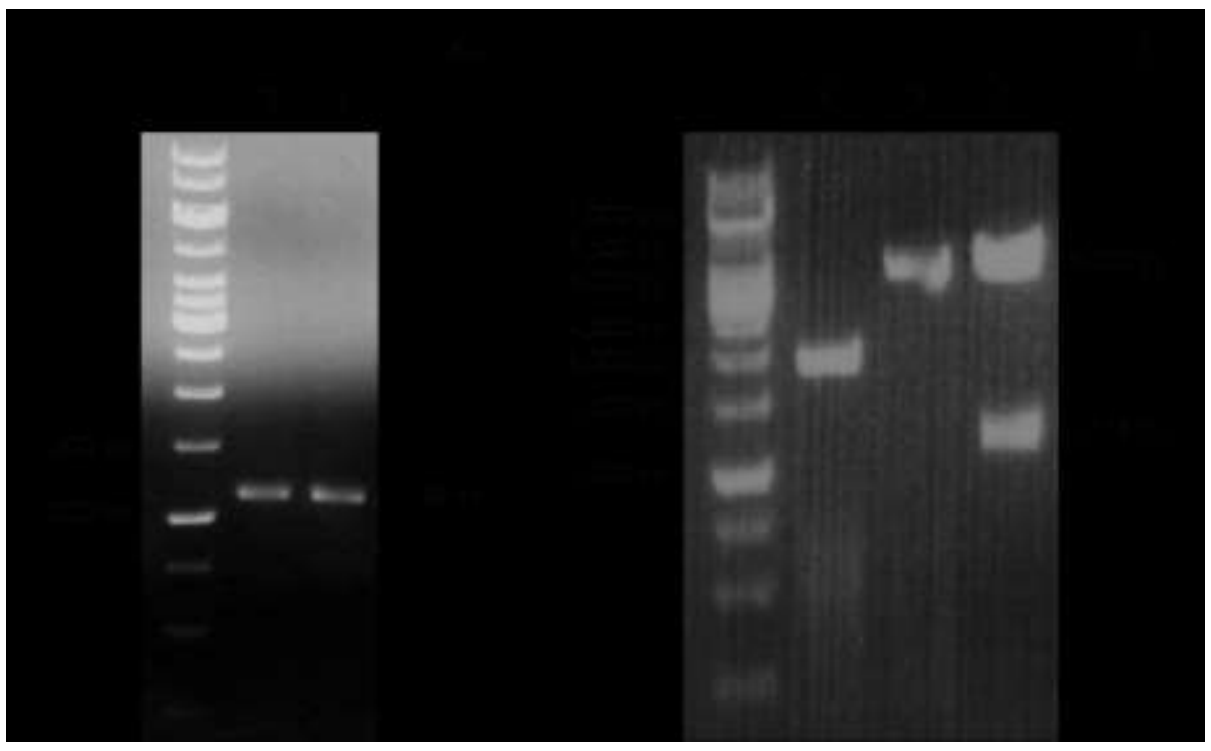

Figure 30. Amplification of the $C g E C M 33$ gene and verification of the generated pQE30:CgECM33plasmids

A: PCR products of $C g E C M 33$ gene. Lane 1: DNA marker, lane 2 and 3: 1,150 bp of $C g E C M 33$. $\mathrm{B}$ : verification of the generated $\mathrm{pQE} 30: \mathrm{CgECM} 33$ plasmids by using double restriction enzyme digestion. Lane 1: DNA marker, lane 2: circular pQE30:CgECM33 plasmid, lane 3: pQE30 digested with BamHI/PstI, lane 4: pQE30:CgECM33 plasmid digested with BamHI/PstI. 


\subsection{2 $C g E \mathrm{Em} 33$ protein expression in $E$. coli}

Recombinant $C g E \mathrm{Ecm} 33$ protein was expressed in $E$. coli for production of anti-Ecm33 polyclonal sera in rabbits. The recombinant $C g E \mathrm{Em} 33$ protein produced in $E$. coli was used for immunization rather than the variant produced in P. pastoris because of its lack of glycosylation circumventing production of non-specific antibodies to carbohydrate components. The transformed E. coli was induced with $1 \mathrm{mM}$ IPTG for 4-5 hours and the His-Tag affinity chromatography was used to purify the $C g E \mathrm{Em} 33$ protein from cell lysates.

During purification, a band corresponding to recombinant $C g E c m 33$ protein was not found in the flow through or the washing steps (Figure $31 \mathrm{~A}$ ). Instead, the recombinant $\mathrm{CgEcm} 33$ was released from the Ni-NTA column in subsequent elution steps 3 and 4 (Figure 31 B).
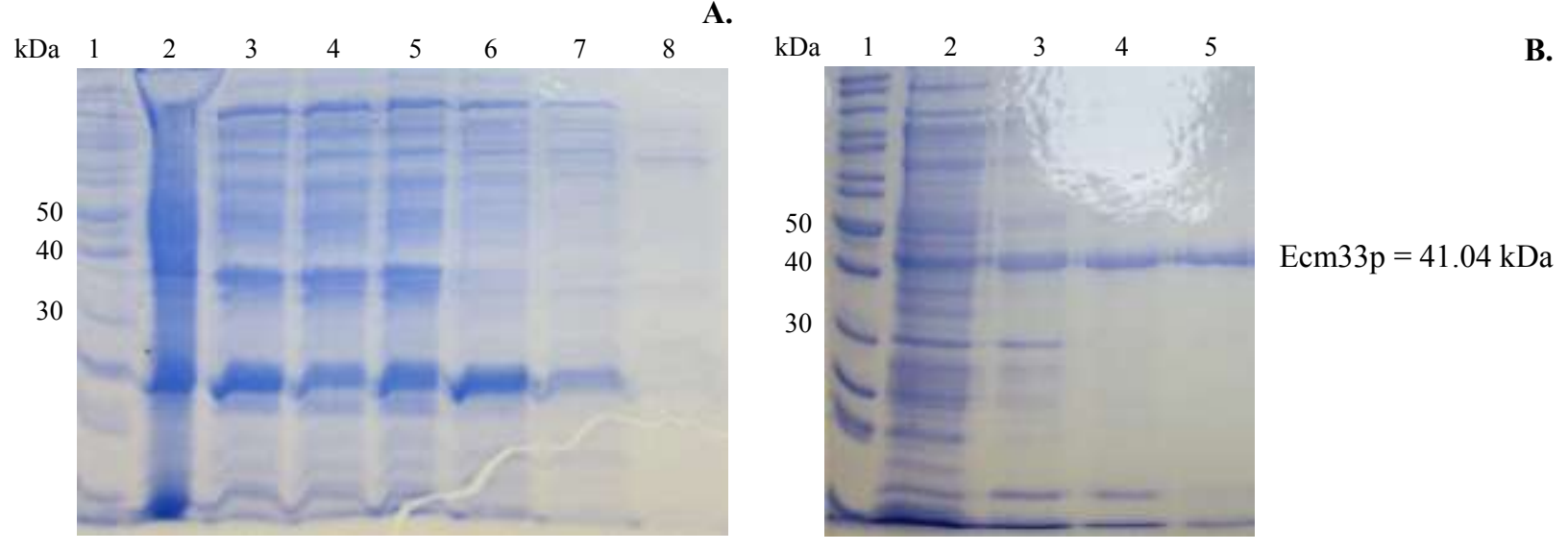

Figure 31. Purification of recombinant $C g E \mathrm{E} \mathrm{B3}$ by His-tag affinity chromatography

A: purification steps; lane 1: protein marker, lane 2: supernatant, lane $3-4: 1^{\text {st }}$ and $2^{\text {nd }}$ flow through, and lane 5-8: $1^{\text {st }}$ to $4^{\text {th }}$ washing. There is no an expected protein band in all steps.

B: elution steps; lane 1: protein marker, lane 2-5:1-4 elution, the expected $C g E c m 33$ protein band was encountered in all elution steps but the $C g E \mathrm{Ecm} 33$ in $4^{\text {th }}$ elution was purest. 


\subsubsection{Anti-Ecm33 antibody production}

Production of anti-Ecm33 polyclonal antisera was performed by Eurogentec, Liège, Belgium. The purified recombinant $C g E c m 33$ protein produced in $E$. coli was immunized in two rabbits (code number 22 and 23) by using $100 \mu \mathrm{g}$ in each immunization. Serum samples of both animals were analysed before immunization to exclude the preexistence of antibodies cross reacting with $C g E c m 33$ (data not shown). Serum samples were tested for antibody production at days 0 (pre-immune serum), 38, 66, and 87 after the first immunization by Western blott analysis with the recombinant $C g E \mathrm{Em} 33$ proteins produced in $E$. coli as well as P. pastoris. In addition, the recombinant $C g$ Pst1 proteins were used to analyse a cross-reaction. The results demonstrated that the generated polyclonal anti-Ecm33 antibodies can recognize both recombinant Ecm33 proteins from E. coli and P. pastoris as shown in Figure 32. Moreover, cross reaction of the antiEcm33 polyclonal antibody from rabbit no. 23 to the Pst1 protein was observed (Figure 32 B).

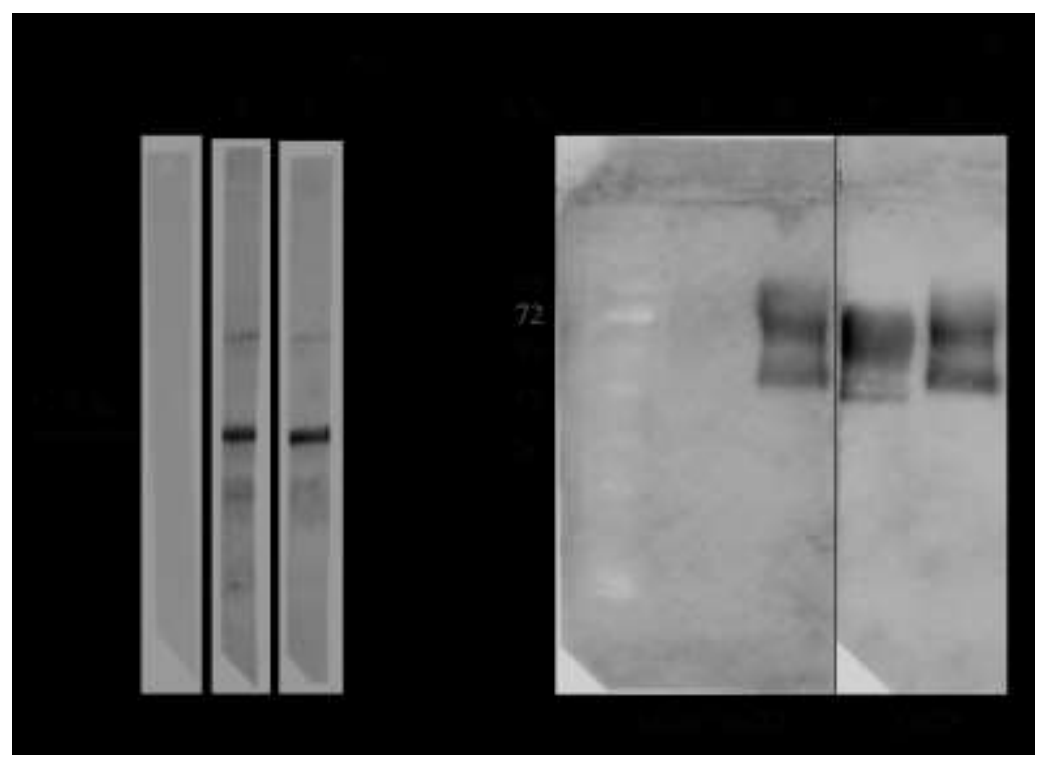

Figure 32. Western blot analyses of the generated anti-CgEcm33 antisera

A: testing of $C g E c m 33$ antigen from $E$. coli and anti-CgEcm33 antisera (titer 1:4,000). Lane 1: pre-immune serum, lane 2: serum in $2^{\text {nd }}$ month of rabbit No.22, lane 3 : rabbit serum in $2^{\text {nd }}$ month of rabbit No.23. The results demonstrate that the immunized serum in $2^{\text {nd }}$ month of two rabbits can recognize $C g E c m 33$ antigen purified from $E$. coli.

B: testing of $C g E c m 33$ and $C g P s t 1$ antigens from $P$. pastoris and anti- $C g E c m 33$ antisera (titer 1:4,000). Lane 1: protein marker, lane 2: $C g$ Pst1 and rabbit serum No.22, lane 3: $C g E c m 33$ and rabbit serum No.22, lane 4: $C g$ Pst1 and rabbit serum No. 23, lane 5: $C g E c m 33$ and rabbit serum No.23. The results demonstrate that serum from rabbit no. 22 can recognize $C g E \mathrm{~cm} 33$ only but serum from rabbit no. 23 can recognize both $C g E c m 33$ and $C g P s t 1$. 


\subsection{Cell wall protein release assay}

To investigate a potential loss of cell wall components in the absence of the Ecm33 protein overnight culture supernatants were collected from C. glabrata wild-type and mutant strains and concentrated 50 -fold (15 ml spin column pore size 10,000 Da). In the concentrated culture supernatants total protein concentrations were measured and SDS-PAGE and western blot analyses performed to determine a potential release of $\beta-1,6$-glucan and/or the abundant glucan-bound structural cell wall protein Cwp1.

Compared to the wild-type, the $C$. glabrata $\Delta e c m 33$ mutant released both, 1,6- $\beta$-glucan

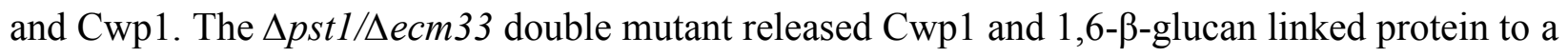
larger degree than $\Delta e c m 33$ only (Figure 33).

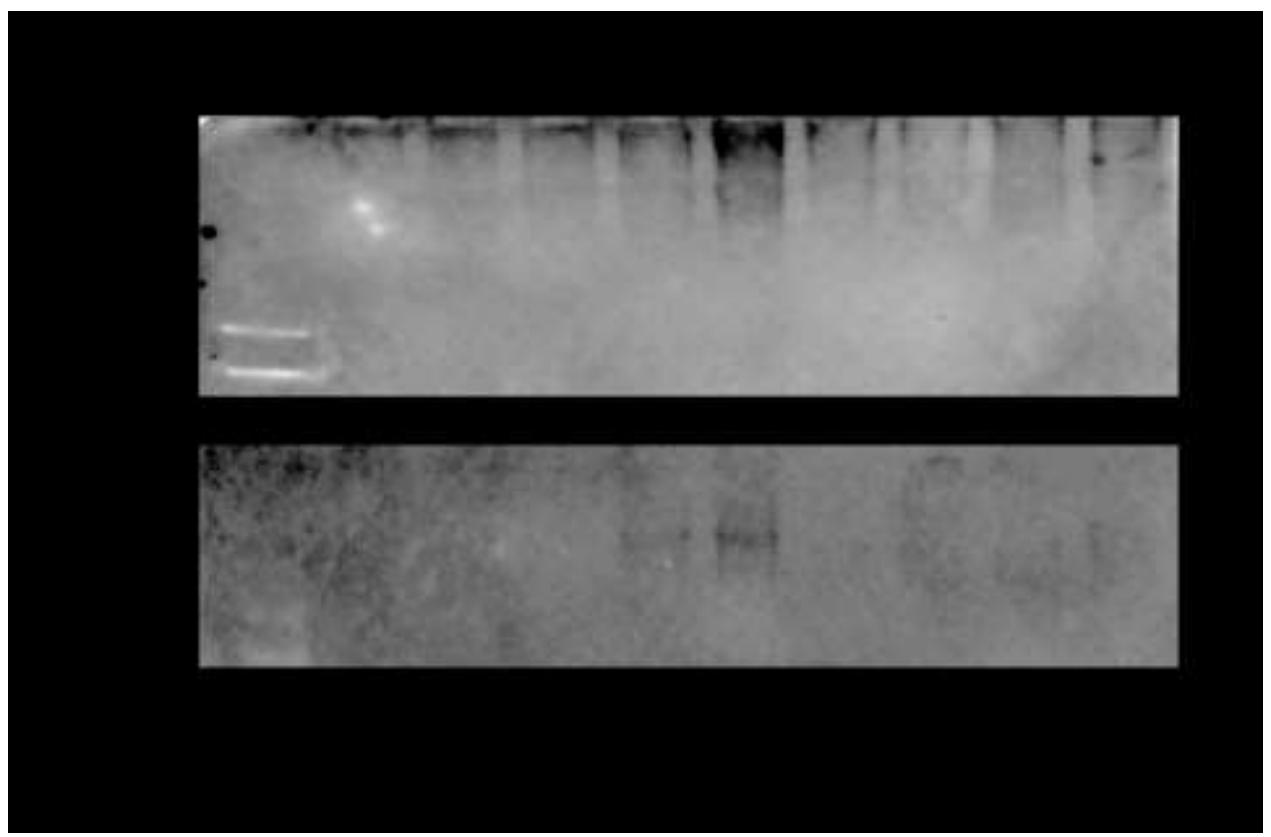

Figure 33. Western blot analyses of culture supernatants

A: Detection of 1,6- $\beta$-glucan linked protein in culture supernatants by western blot with monoclonal rabbit anti- $\beta$-1,6-glucan antibodies. Note that glucans do not migrate inside the separating gel due to their size, but are rather stacked at the top. B: Detection of Cwp1 protein in culture supernatants by western blot with polyclonal rabbit anti-Cwp1 antibodies.

Lane 1: protein marker, lane 2: concentrated culture supernatant of wild-type, lane 3: $\Delta h t l$ (auxotroph), lane 4: $\Delta p s t 1$, lane 5: $\Delta e c m 33$, lane 6: $\Delta p s t 1 / \Delta e c m 33$, lane 7: $\Delta p s t 1: C g P S T 1$, lane 8: Aecm33:CgECM33, lane 9: $\Delta p s t 1 / \Delta e c m 33: C g P S T 1$, lane 10: $\triangle p s t 1 / \Delta e c m 33: C g E C M 33$

${ }^{\mathrm{a}}$ : ratio calculated by the formular of (percent of total protein release $\div$ percent of growth rate) $\mathrm{x}$ 100 ; growth rate and total protein release was relative value normalized to the auxotrophic wildtype strains. 


\subsection{Binding assay}

As the molecular function of the Ecm33 protein family members is unknown, a binding assay was performed to investigate whether Ecm33 might actively bind to the cell wall.

The results demonstrated that both recombinant Ecm33 and Pst1 proteins bind better to the extracted cell walls (ECWs) of the $\Delta e c m 33, \Delta e c m 33: t r u n c a t e d ~ C g E C M 33$ and $\Delta p s t 1 / \Delta e c m 33$ mutants as compared to wild-type and $\Delta p s t 1$ ECWs (Figure 34 ).

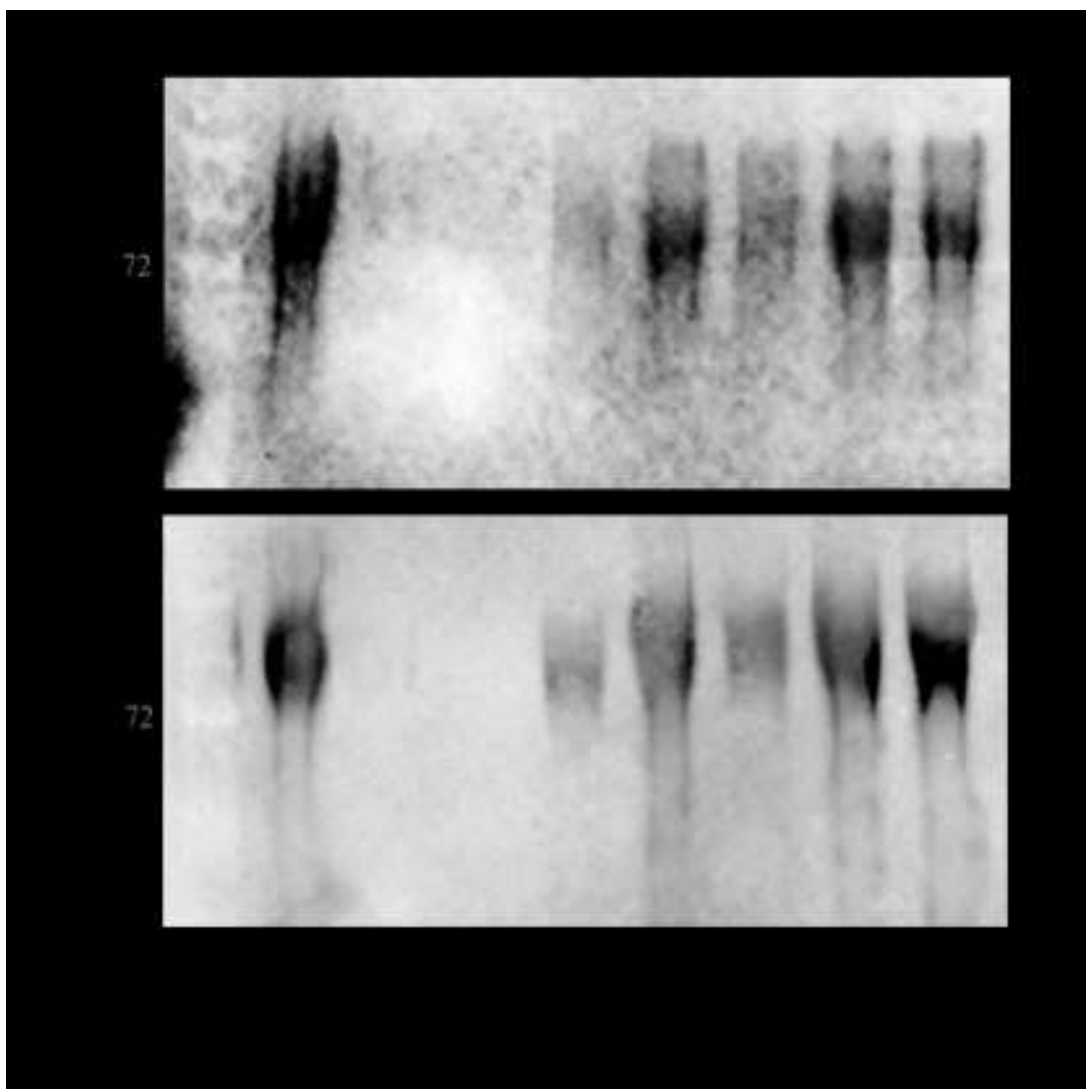

Figure 34. Binding assay

A: Incubation of the extracted cell walls (ECWs) and Pst1 protein, detection with rabbit serum no. 23.

B: Incubation of the extracted cell walls (ECWs) and Ecm33 protein, detection with rabbit serum no. 22.

Lane 1: protein marker, lane 2: Pst1 protein from P. pastoris in A. or Ecm33 protein from $P$. pastoris in B., lane 3: wild-type ECWs, lane 4: ELISA blocking solution, lane 5: wild-type ECWs, lane 6: $\Delta e c m 33 \mathrm{ECWs}$, lane 7: $\Delta p s t 1 \mathrm{ECWs}$, lane 8: $\operatorname{eccm33}$ :truncated $C g E C M 33 \mathrm{ECWs}$, lane 9: $\Delta p s t 1 / \Delta e c m 33 \mathrm{ECWs}$ 


\section{CHAPTER 4}

\section{DISCUSSION}

The increased incidence of fungal infections seen over the past decades significantly impacts on human public health (Giri, S. and Kindo, A. J., 2012; Mayer, F. L., Wilson, D. et al., 2013). Among fungal infections, candidiasis is the most frequent and C. glabrata is the second most prevalent causative agent of systemic and local candidiasis. C. glabrata infection may become difficult to treat because this organism can be highly resistant to anti-mycotic drugs, especially the commonly used substance fluconazole (Borg-von Zepelin, M., Kunz, L. et al., 2007; Pfaller, M. A., Diekema, D. J. et al., 2010). The mortality rate of candidiasis caused from C. glabrata infection can be as high as $50 \%$ in patients with extended candidemia longer 30 days (Das, I., Nightingale, P. et al., 2011).

For fungal pathogenesis, the fungal cell wall carries key functions. Among proteins localized to the C. glabrata cell wall, the a glycosyl-phosphatidylinositol (GPI)-anchored protein Ecm33 is one of the most abundant (de Groot, P. W., Kraneveld, E. A. et al., 2008). Sequence homologs of Ecm33 can be found in many fungi, where full genome sequences are available. Examples are not only budding yeasts, but also fission yeast, dimorphic fungi, dermatophytes, other molds, and plant pathogenic fungi.

Previous studies showed that Ecm33 proteins was associated with cell wall integrity an and biofilm formation, and pathogenicity (Martinez-Lopez, R., Monteoliva, L. et al., 2004; Pardo, M., Monteoliva, L. et al., 2004; Chabane, S., Sarfati, J. et al., 2006; Martinez-Lopez, R., Park, H. et al., 2006; Romano, J., Nimrod, G. et al., 2006; Martinez-Lopez, R., Nombela, C. et al., 2008). Experimental evidence also suggested differences in the biological role of Ecm33 between, for example, yeast and molds. Furthermore, the exact biochemical function of Ecm33 in any fungus remains speculative. Because of the clinical impact of the cell wall and the fact that Ecm33 homologs were identified experimentally in the cell wall proteome as very abundant CWPs in the human pathogen C. glabrata (de Groot, P. W., Kraneveld, E. A. et al., 2008), this work was performed to characterize the role of the Ecm33 protein family in this organism and 
the results were compared to comprehensive analyses that were carried out with the homologs in C. albicans and $S$. cerevisiae.

\section{1 $C \mathrm{gECM} 33$ is required for cell fitness, cell wall integrity, and adhesion}

The results from agar drop dilution assays using different cell wall perturbing agents demonstrated that the $\Delta e c m 33: \Delta e c m 33$ strain of $C$. albicans and the $\Delta e c m 33$ and $\Delta p s t 1 / \Delta e c m 33$ strains of $S$. cerevisiae were sensitive to Caffeine, Calcofluor white, and Congo red when compared to the auxotrophic wild-type in each organism. However, there were no phenotypic differences in the $\Delta p s t 1$ mutant of $S$. cerevisiae compared to the wild type strain. Our results confirm previous studies which showed that the $\Delta e c m 33$ mutant in C. albicans was susceptible to Calcofluor white, Congo red and Hygromycin B (Martinez-Lopez, R., Monteoliva, L. et al., 2004) and $\Delta e c m 33$ and $\Delta p s t 1 / \Delta e c m 33$ mutants in S. cerevisiae had a cell wall weakness, being sensitive to Calcofluor white, Caffeine and Hygromycin B (Pardo, M., Monteoliva, L. et al., 2004). These results indicate that ECM33 of C. albicans and S. cerevisiae are required for cell wall integrity.

Surprisingly, all generated mutants in C. glabrata did not show the phenotypic differences which were discovered in $C$. albicans and $S$. cerevisiae using agar drop dilution assays. However, we were able to demonstrate that this observation is based on technical limitations of these assays in this organism, as C. glabrata shows a high intrinsic resistance towards these substances. Instead, the $\Delta e c m 33$ and $\Delta p s t 1 / \Delta e c m 33$ mutants in C. glabrata show phenotypes when higher concentrations of cell wall perturbing agents are used. Therefore, additional broth micro-dilution assays were developed to explore the generated mutant strains in C. glabrata. These assays showed that the $\Delta e c m 33$ mutant in C. glabrata was more susceptible to Calcofluor white and Congo red. The $\Delta p s t 1 / \Delta e c m 33$ double mutant was even more susceptible (approx. 4-times more sensitive when compared to the wild-type) towards Calcofluor white, Congo red, SDS and Hygromycin B. Taken together, these results indicate that ECM33 in $C$. glabrata is also required for cell wall integrity.

The $\Delta e c m 33$ mutants in $C$. albicans, $S$. cerevisiae and $C$. glabrata had a defect in growth under YPD and RPMI 1640 media at $30^{\circ} \mathrm{C}$ and $37^{\circ} \mathrm{C}$ condition. The completed revertant of $C$. albicans and $C$. glabrata showed normal growth as the Caf2 and 4 htl auxotroph strains, 
respectively. These data indicate that ECM33 is an essential protein for growth in the three organisms. Moreover, we found that the $\Delta p s t 1 / \Delta e c m 33$ double mutant in $C$. glabrata and $S$. cerevisiae had slower growth rates than the $\Delta e c m 33$ mutant in both media and at both temperatures. These data indicate synergistic effects upon deletion of ECM33 and PST1. However, no significant differences of growth were observed in the $\Delta p s t 1$ mutant of S. cerevisiae and C. glabrata when compared to its auxotrophic wild-type. Taken together, Ecm33 is a key protein in the cell wall of $C$. glabrata that determines not only the cell wall strength, but also influences the growth rate of these organisms.

Furthermore, the analyses on the generated C. glabrata strains showed that the 4 ecm 33 mutant displays significantly increased hydrophilic surface properties when compared to the wild-type or auxotrophic wild-type strain. Also, the $\Delta p s t 1 / \Delta e c m 33$ double mutant was found to be more hydrophilic as compared to the $\Delta e c m 33$ single mutant. The results from polystyrene adherence capacity assays correlated with hydrophobicity surface properties of the strains: more hydrophilic (less hydrophobic) isolates showed less polystyrene adherence capacity and consequently, the C. glabrata mutants $\Delta e c m 33$ and $\Delta$ pst1/Aecm33 lost polystyrene adherence capacity. In contrast, no phenotypic alterations were observed in terms of hydrophilicity and/or polystyrene adherence capacity in the $C$. albicans and $S$. cerevisiae mutants. However, it should be noted that the parental strains that were used to generate the mutants in $C$. albicans and $S$. cerevisiae do not have any ability to adhere to polystyrene. Therefore, it is difficult to compare and correlate ECM33 function and the influence on polystyrene adherence in these organisms.

The results of silicone adherence assays demonstrated that the $\Delta e c m 33$ mutant of $C$. glabrata has a lower adherence capacity to this material as compared to the wild-type strain. The $\Delta p s t 1 / \Delta e c m 33$ double mutant is reduced approximately $60 \%$ when compared to wild-type and also shows a significant reduction when compared to the $\Delta e c m 33$ mutant. The $\Delta e c m 33$ mutant of C. albicans is also affected in silicone adherence capacity, but this phenotype was not observed in the $S$. cerevisiae mutants.

The results obtained with $C$. glabrata confirm that ECM33 affects important adherence capacities of human pathogenic Candida spp. and this assumption is further supported by our results with the $C$. albicans mutants and a previous study using a $C$. albicans $\Delta$ ecm 33 mutant. This study showed that in $C$. albicans the $\Delta e c m 33$ mutant displays a lower adherence and reduced invasion to endothelial and epithelial cells as compared to auxotrophic wild-type 
(Martinez-Lopez, R., Park, H. et al., 2006; Rouabhia, M., Semlali, A. et al., 2012). Therefore, we conclude that beyond cell wall strength and growth, ECM33 contributes significantly to the adherence capacities of human pathogenic yeasts. As a consequence ECM33 might be considered a gene associated with virulence in Candida spp.

\subsection{ECM33 of C. glabrata, C. albicans, and $S$. cerevisiae have a similar function but not identical functions.}

To explore the function of $\mathrm{CgEcm} 33$-family a set of complementation mutants were generated and analyzed. In the CgAecm 33 strain (i) the homologs of C. albicans (CaECM33) and S. cerevisiae (ScECM33), (ii) the ECM33 homologs of C. glabrata (PST1, SPS2 and SPS22) and (ii) a truncated version $C g E C M 33$ without the GPI-anchor motif were introduced in order to explore if the phenotypes of the $\mathrm{Cg} \Delta$ ecm 33 mutant can be rescued. All generated strains were tested for growth, cell wall strength, hydrophobicity and adhesion to polystyrene and silicone. Gene expression analysis confirmed that in both constructed strains, Aecm33:CaECM33 and Aecm33:ScECM33 could be expressed at a levels that compare to approx. $90 \%$ of the wild-type ECM33 gene in C. glabrata. Interestingly, the phenotypes of the two complemented strains were very similar to the $C$. glabrata wild-type, indicating that both heterologous homologs can compensate the role of $C g E C M 33$ in terms of cell wall fitness, growth and cell wall composition. However, in the adherence assays and the Alcian blue staining assay, the phenotypes of the CgAecm33 mutant could not fully be rescued on all experiments. This might indicate that subtle differences do exist in the multilayered function of the ECM33 genes that manifest e.g. in the assembly and/or incorporation of cell wall proteins (mediating negative surface charge) including the cell wall adhesins. Another option would be that the Ecm33 protein acts as a adhesion protein itself, but this seems to be not very likely as the S. cerevisiae Aecm 33 mutant expressing CaECM33 does not adhere to or penetrate epithelial cell whereas the auxotrophic wild type strain of C. albicans does (Martinez-Lopez, R., Park, H. et al., 2006).

The next question was whether the homologous genes of the CgECM33 family can replace the function of $C g E C M 33$. To answer this question, a second set of overexpression

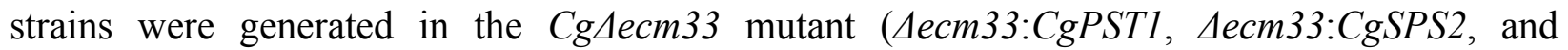
Aecm33:CgSPS22) and tested as described above. The results of gene expression analyses 
showed that there were significantly increased levels of up-regulation in the three overexpression strains, but the expression levels of the integrated genes were found only $20 \%, 15 \%$ and $10 \%$, respectively above the wild-type level. We also excluded that a negative feedback regulation is observed in the ECM33 gene family of C. glabrata and that the gene product from an introduced over-expression plasmid induces the down-regulation of the natural expression of the respective gen homologs. Moreover, the phenotypic results from the three complemented strains displayed very similar phenotypes as the $\Delta e c m 33$ mutant indicating, that the other three members of this family can not compensate the function of $C g E C M 33$. These data support the idea that the proteins do not show functional redundancy. However, at this point it cannot be ruled out, that the experimental level of overexpression was too low to induce noticeable compensatory effects or that a few overlapping, but so far unrecognized activities do exist within this family. In agreement with these data in a previous study in $S$. cerevisiae high level expression of ScPST1 in a ScAecm33 mutant (Aecm33/Yep352-PST1 strain) could not compensate or rescue the phenotype of the $\Delta e c m 33$ strain, when tested with the cell wall perturbing agents Calcofluor white, Congo red and Caffeine. However, a very slight compensation was observed for the hygromycin B phenotype (Pardo, M., Monteoliva, L. et al., 2004).

When the truncated version of $\mathrm{CgECM} 33$ without the GPI-anchor motif was reintroduced in the $C g \Delta E c m 33$ mutant all the phenotypes observed in the deletion strain could not be rescued. This confirms that the anchor and most likely covalent cell wall incorporation of ECM33 is necessary for its proper function (see below).

\subsection{Ecm33 is associated with alterations in the chemical composition of the cell wall}

As outlined above, the results of the Alcian blue staining experiments showed that ECM33 deletion (Aecm33 and $\triangle p s t 1 / \Delta e c m 33$ ) resulted in an increase of negative charges on the cell surface of $C$. glabrata pointing to an altered composition of the cell wall (possibly mediated by macromolecules such as proteins, mannans, glucans, and chitins). In contrast, this effect was not observed when only PST1 was deleted in C. glabrata.

To further investigate these compositional alterations staining of glucan and chitin in the yeast cells walls using wheat-germ agglutinin (WGA-Alexafluor 647 conjugate) and Congo red, respectively were performed and analyzed by using a Flow cytometer. The results of the chitin 
staining experiments in the three organisms demonstrated that the $\Delta e c m 33$ and $\Delta p s t 1 / \Delta e c m 33$ mutants, but not the $\Delta p s t 1$ mutant, had more chitin in cell wall when compared to wild-type auxotrophic strain. Again, when the homologs of the CgECM33-family were over expressed in the C. glabrata $\triangle e c m 33$ mutant ( $\triangle e c m 33: C g P S T 1, \triangle e c m 33: C g S P S 2$, and $\triangle e c m 33: C g S P S 22)$ a reduction of the level of chitin was not observed showing that this alteration in cell wall composition can not be rescued by these ECM33 family members. In contrast, ECM33 from $C$. albicans and $S$. cerevisiae could complement this phenotype in C. glabrata. The observed increase in the chitin level of the mutant might have different reasons: (i) ECM33 might be involved in the incorporation of chitin in the cell wall. Consistent with this, a previous report on C. albicans showed that $\Delta c h s$ (chitin synthase) 2, $\Delta c h s 3, \Delta c h s 2 / \Delta c h s 3$, and $\Delta e c m 33$ mutants could not release melanin pigment to the environment after induction with L-3,4dihydroxyphenylalanine (L-DOPA) and $N$-acetylglucosamine, while the auxotroph NGY152 could do that. This indicates that a functional ECM33 gene is required for the production of cell wall chitin components involved in melanin externalization (Walker, C. A., Gomez, B. L. et al., 2010). These analyses were also performed with the generated C. glabrata strains, however no melanin externalization, neither in wild-type strains, nor in the mutants was observed in this organism (data not shown). (ii) The increased chitin might also reflect an indirect and compensational effect induced by the cell wall weakening in the $\mathrm{Cg} \Delta \mathrm{ecm} 33$ mutant. Generally, when fungi face a disruption of cell wall strength, an up-regulation of the cell wall integritypathway and subsequently a compensatory increase in chitin production is observed to prevent cell wall collapse (Kapteyn, J. C., Van Egmond, P. et al., 1999). In agreement with our findings, in two previous studies, S. cerevisiae Aecm33 mutants display an enhanced Calcofluor white fluorescence intensity due to high chitin content on cell wall (de Groot, P. W., Ruiz, C. et al., 2001; Pardo, M., Monteoliva, L. et al., 2004).

The relative quantification of the glucan level in the cell wall exhibited no significant differences between any of the C. glabrata strains. Also alterations of the glucan level in cell wall were not observed in the mutants of $C$. albicans and S. cerevisiae. These data imply that the lack of ECM33 does not affect the total glucan level on the cell wall.

However, using Western blot analyses we observed that in $\mathrm{Cg} \Delta$ ecm 33 mutant released more $\beta$-1,6-glucan linked protein to the culture supernatant as compared to wild-type strain and this effect was even more pronounced in the double $\Delta$ pst1/4ecm 33 mutant. This significant 
observation was also made in the ScAecm33 mutant (de Groot, P. W., Ruiz, C. et al., 2001). In addition, the abundant cell wall protein Cwp1 of C. glabrata was detected in the culture supernatant of $\Delta e c m 33$ and $\Delta p s t 1 / \Delta e c m 33$ mutants using Western blot analyis. This result is similar to the result of a study by Pardo et al. in $\Delta e c m 33$ mutant of $S$. cereviaise. This group showed that the $\mathrm{ScCwp} 1$ protein is released to the culture medium in the bakers yeast mutant. In contrast, in a further study by De Groot's group the $S c \mathrm{Cwp} 1$ protein was not identified in the culture supernatant of $\Delta e c m 33$ mutant of $S$. cereviaise. However, both groups noticed the release of Pir2 cell wall proteins, $\beta$-1,6-glucan (Pardo, M., Monteoliva, L. et al., 2004) and also $\beta$-1,3glucan (de Groot, P. W., Ruiz, C. et al., 2001) to the culture supernatant. In summary, the altered cell wall composition and the loss of cell wall components and proteins of the mutants indicate that ECM33 plays crucial role in the assembly of the cell wall in C. glabrata influencing its architecture and function.

\subsection{A model for the role of Ecm33 for the fungal cell wall}

As shown above, deletion of ECM33, but not PST1, has a profound effect on the stability and architecture of the cell wall, not only in $C$. glabrata, but also in other fungi such as $C$. albicans and $S$. cerevisiae. The cells are significantly more susceptible to cell (wall) perturbing agents, reflecting a loss of cell wall rigidity. Furthermore, the composition of the cell wall is altered in a way that more chitin is present (potentially representing a compensatory mechanism), and $\beta-1,6$-glucan containing material is lost to the environment. Most likely as a consequence of $\beta$-1,6-glucan loss, also adhesion is reduced, since adhesins are in turn linked to $\beta$-1,6-glucan through GPI-anchors. In total, this leads to reduced fitness, and probably also to reduced virulence, as shown at least for C. albicans (Martinez-Lopez, R., Monteoliva, L. et al., 2004; Martinez-Lopez, R., Park, H. et al., 2006; Martinez-Lopez, R., Nombela, C. et al., 2008; Rouabhia, M., Semlali, A. et al., 2012).

These phenotypes can be explained, if we assume that Ecm33 provides a crucial link between $\beta$-1,6-glucan and another, as of yet unknown, component of the cell wall (Figure 35). 


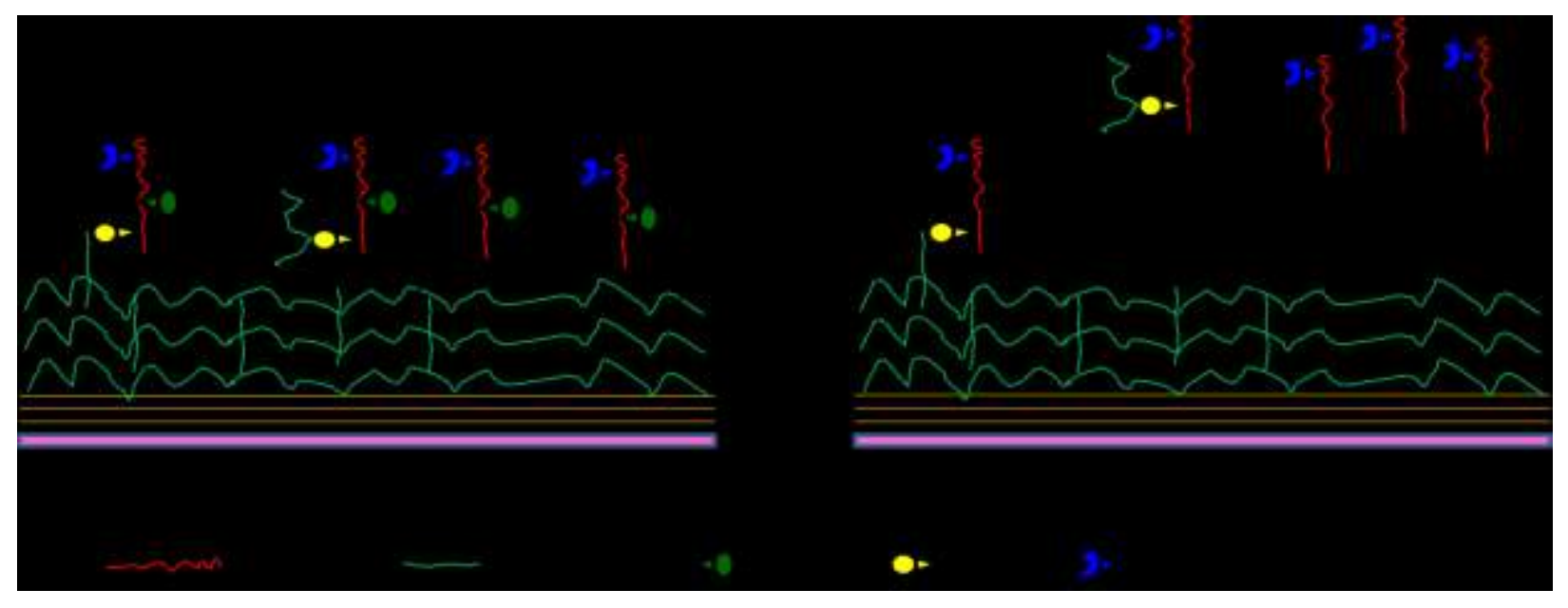

Figure 35. Hypothetical model of Ecm33 contribution to cell wall structure

\subsection{Exploring the molecular function of Ecm33}

Three possibilities of Ecm33 protein function can be proposed based on our experimental results: Model I: Ecm33 could be a structural protein, where Ecm33 is linked to the cell wall through the GPI anchor and the activity of another protein; Model II: Ecm33 could be a lectin, binding carbohydrate moieties of the cell wall; Model III: Ecm33 itself could be an enzyme, linking 1,6-glucan containing material to the cell wall (Figure 36).

To explore these options, different molecular tools were set up to help deducing data that support any of the model proposed above: active recombinant forms of Ecm33 and Pst1 proteins were made in $P$. pastoris, antisera were raised against recombinant Ecm33 expressed and purified from E. coli, and to explore the role of the GPI-anchor, C. glabrata Aecm33 strain expressing a C-terminally truncated form of Ecm33 (devoid of the GPI anchor) were created. 


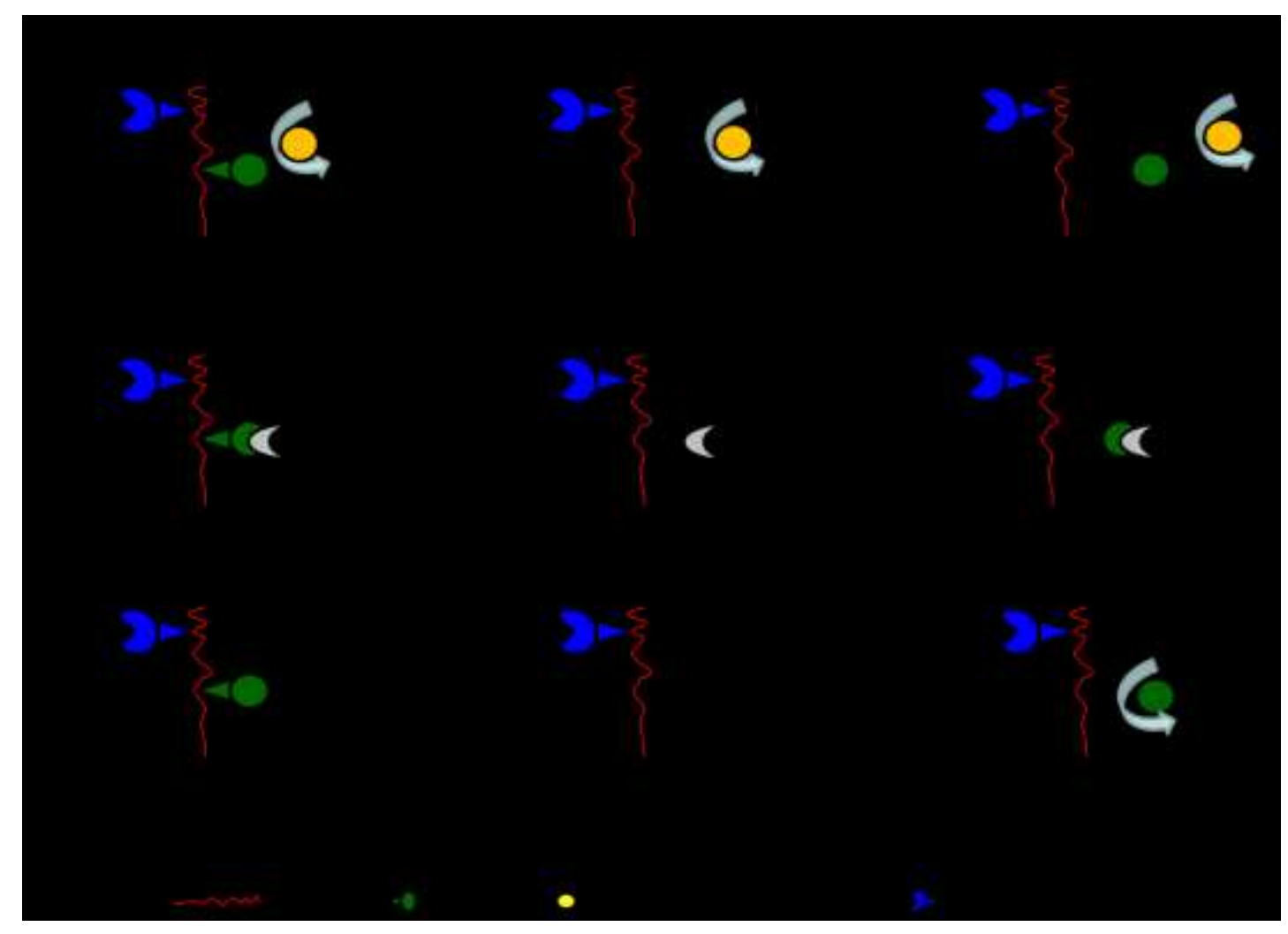

Figure 36. Models of potential Ecm33 functions

If Ecm33 was an enzyme, the GPI anchor should not be needed for full function. We therefore re-investigated all of the phenotypes observed in the $\Delta e c m 33$ mutant in a strain expressing a truncated, GPI-anchor-less form ( $\triangle e c m 33$ :truncated CgECM33). As described above, the phenotypic results of $\triangle e c m 33$ :truncated $C g E C M 33$ were similar to $\triangle e c m 33$ mutant while the strain $\triangle e c m 33: C g E C M 33$ was conformable to wild-type, indicating that a truncated form cannot, even partially, complement the loss of ECM33. This strongly argues against an enzymatic function of Ecm33. It also implies that the GPI-anchor of Ecm33 protein is a necessary part for its function.

In case that, ECM33 was a structural component or a lectin, the protein should be found in the cell wall of $\triangle e c m 33$ :truncated $C g E C M 33$ despite the lack of its GPI anchor. In the case of a structural component, Ecm33 should be covalently linked, and not extractable, in the case of a lectin it should bind the cell wall reversibly. To address this, antisera against recombinant Ecm33 produced in $E$. coli were raised. However, although antisera from two animals vividly recognized 
the recombinant form of the protein, they failed to recognize any epitope in proteins or cell walls extracted from C. glabrata.

Nevertheless, the antisera were able to recognize recombinant Ecm33 produced in $P$. pastoris. One of the antisera also cross-reacted with recombinant Pst1, enabling the study of both proteins. Binding assays of the two recombinant proteins were carried out on extracted cell walls (ECWs) from the different mutant strains. These experiments demonstrated that $C g E c m 33$ and $C g$ Pst1 protein both bind ECWs from $\Delta e c m 33, \Delta e c m 33$ :truncated $C g E C M 33$ and $\Delta p s t 1 / \Delta e c m 33$ better than wild-type and $\Delta p s t 1$ ECWs. From this, we can propose that the lack of Ecm33, but not Pst1 protein in the cell wall leaves epitopes which can be actively and reversibly bound by both Ecm33 and Pst1.

In order to identify potential binding partners, initial competition binding experiments with glucose and N-acetylglucosamine (Supplements, chapter 6.4) were carried out. Preliminary results from these experiments suggest a stronger inhibition of Ecm33 binding to the cell wall by glucose than $\mathrm{N}$-acetylglucosamine.

According to our results, it can be summarized that Ecm33 in C. glabrata is associated with cell growth, cell wall assembly and integrity, chemical composition of the cell wall and the adherence capacity of the organism. The complementation experiments show that the Ecm 33 proteins form $C$. albicans, $S$. cerevisiae and C. glabrata have overlapping, but not identical functions, as many phenotypes that were observed in the $\mathrm{Cg} \Delta$ ecm 33 mutant could be restored by heterologous expression. Interestingly, the adherence phenotype and the altered surface charge could not be complemented by heterologous expression indicating that $C g E c m 33$ shows subtle functional differences possibly in mediating the proper incorporation of cell wall proteins by acting as a lectin. The results also show that the other members of the Ecm33 protein family in C. glabrata (Pst1, Sps2, and Sps22) are functionally not redundant and are not upregulated in response to ECM33 deletion. 


\section{CHAPTER 5}

\section{SUMMARY}

Candida glabrata is the second most frequent cause of local and systemic human candidiasis and infections with this species are difficult to treat because of frequent azole resistance. C. glabrata infections have high mortality rates in immunocompromised patients. During pathogenesis, the cell wall is of particular importance, because it holds key functions such as adhesion and countering of immune defenses.

Next to the structural proteins of the Cwp1-family, Ecm33 and Pst1 have been identified as the most abundant GPI-anchored proteins in the cell wall, but their functions are largely unclear. In $C$. albicans and $S$. cerevisiae, the ECM33 deletion mutants show partially overlapping phenotypes suggesting defects in cell wall assembly. Therefore, gene disruption and complementation experiments were conducted on these genes in C. glabrata.

The results of these experiments show that, similar to $C$. albicans and $S$. cerevisiae, the C. glabrata $\Delta e c m 33$ and $\Delta p s t 1 / \Delta e c m 33$ mutants are susceptible to most cell wall perturbing agents. The mutants have a more negatively charged cell wall surface and release Cwp1 protein and $\beta-1,6$-glucan containing material to the environment. Most importantly, as a consequence the mutants lose some adherence capacities, which is of particular interest in terms of C. glabrata pathogenicity. With the notable exceptions of the adherence phenotype and the surface charge, the phenotypes are rescued by introduction of ScECM33 and CaEMC33. In contrast, the phenotypes could not be compensated in the $\Delta e c m 33$ mutant of $C$. glabrata by overexpression of the remaining members of the C. glabrata ECM33-family (CgPST1, CgSPS2 and CgSPS22). However, we can not rule out that this is due to technical limitations of the experimental approaches. From the overall phenotypic data, we can propose that Ecm 33 probably is involved in the linkage of $\beta-1,6$-glucan to the cell wall. If $\beta-1,6$-glucan is lost, so is its carrier function for effector proteins, resulting in the observed phenotypes. The generated phenotypic data also enabled to develope different hypothetical models for the biological function of Ecm33 in $C$. glabrata and the functional analyses performed so far indicate that $C g E c m 33$ might be a lectin. 
These analyses now make it possible to further characterize the biological role of this very abundant cell wall protein, for example using comprehensive binding assays, glycochip experiments and proteomic work on the cell wall and secreted proteomes of the generated mutants. The improved understanding of the cell wall in C. glabrata will enable to characterize pathogenicity in this organism and potentially develop new antifungal therapy. 


\section{CHAPTER 6}

\section{SUPPLEMENTS}

\subsection{DNA sequence of $C g E C M 33$ for cloning in $E$. coli}

DNA sequecnce of $C$. glabrata was taken from online genome database (http://www.genolevures.org) and the restriction enzyme was chosen by checking via NEBcutter V2.0 (http://tools.neb.com/NEBcutter2/).

Online program http://web.expasy.org/translate/ is used to translate DNA sequence to protein sequence. The expected molecular weight of $\mathrm{CgEcm} 33$ protein was calculated from amount of amino acids via http://www.sciencegateway.org/tools/proteinmw.htm. The recombinant $\mathrm{CgEcm} 33$ protein expressed in E. coli consists of 397 amino acids that this protein has a molecular weight as $41.04 \mathrm{kDa}$. The $C g E \mathrm{Em} 33$ protein for cloning in $E$. coli is a partial protein that does not have a signal peptide and GPI anchor.

\section{atgAGAGGATCGCATCACCATCACCATCAC $\underline{\text { GGATCC }}$}

cagaactctacatctgacgatgttccatctgggtgttctcttggtagcagtgctactgctactgcacagtctgatctggacaagtacagcgggt gtgagaccattgttggtaacttgaccatcactggtgctcttggctctgctgcgctagccggtgtcaagaagatcgatggttctttgagaatctac aatgccacctccttgggctcttttgctgctgactccgtcaaggagatcaccggtgctttgaacatgcaggacttgaccatcttgaccactgcgt ccttcggtgcgctggaagaagtcgacaccatcaacttgatcactctgccagccatctccactttcaacactaacttgcaatccgccaacaaca tcgtcgtctccgacacctctttggagtccgtcgaaggtttcggctctttgaaagaggtcaacgtcctaaacatcaacaacaacagatacttgaa ctctttcaagtcctctttggaatccgtgtcaggtgctttgcaattcgcctccaacgccgatgaaaccgccgtgtcctttgacaacttgatctgggc caacaacatcaccttgagagacgtccaaaacgcctctttcgecaagctggagtccgttaacgectccetgggtttcatcaacaacaccatctc cactttgaacttgacccacttgtccaaggtcggtcaatccctatccgttgtctccaacgatgacttgacccagttgtccttcttgaacttgacctcc gtcggcggtggtctggtcgtcgccaacaacactaacttgaagaccatcgacggtttgaagaacgtccaaactgtcggcggtgccatcgaca tcaccggtaacttcaccactttggacttgtcctcettgaagtccgtcagaggtggtgccactttcaacaccgtctccggtaacttctcctgctctc ctttgaagtccctacaaagcaagggtgccatccaaggtgactccttcgtctgcaagaacggtgctacttccacttccatcagcatgtcttccag 
atccegctcttcttccgectcttcctccgettccgctactgttaccgccagatccaacgacactgettccacttcctccaccaagaccaagaagt $\underline{\text { ctCTGCAGCCAAGCTTAATTAGCtga }}$

Annotation; atg and tga: start and stop codon, gray box: 6XHis-tag, gray box: restriction site, black box: primer site, and underline: ECM33 coding sequence

\section{- Protein sequence of $C g E \mathrm{~cm} 33$ expressed in $E . c o l i$}

M R G S H H H H G S Q N T S D D V P S G C S L S S A T A T A Q D L D K Y S G C E T IV G N L T I T G A G S A A L A G V K K I D G S L R I Y N A T S L G S F A D S VKE I T G A L N M Q D L T I L T T A S F G A L E E V D T I N L I T L P A I S T F N T N

L Q S A N N V V S D T S L E S V E G F G S L K E V N V L N I N N R Y L N S F K S L E S V S G A L Q F A S N A D T A V S F D L I W A N N T L R D V Q N A S F A K L E S V N A S L G F I N N T IS T L N L T H L S K V G Q S L S V V S N D D L T Q L F L N L T S V G G G L V V A N N T N L K T I D G L K N V Q T V G G A I D I T G N F T T L D L S S L K S V R G G A T F N T V G NF S C S P L K S L Q S K G A I Q G S F V C K N G A T S T S I S M S S R S R S S S A S S S A S A T V T A R S N D T A S T S S T K T K K S L Q P S L I S 


\subsection{Process of Ecm33 and Pst1 expressed $P$. pastoris generation}

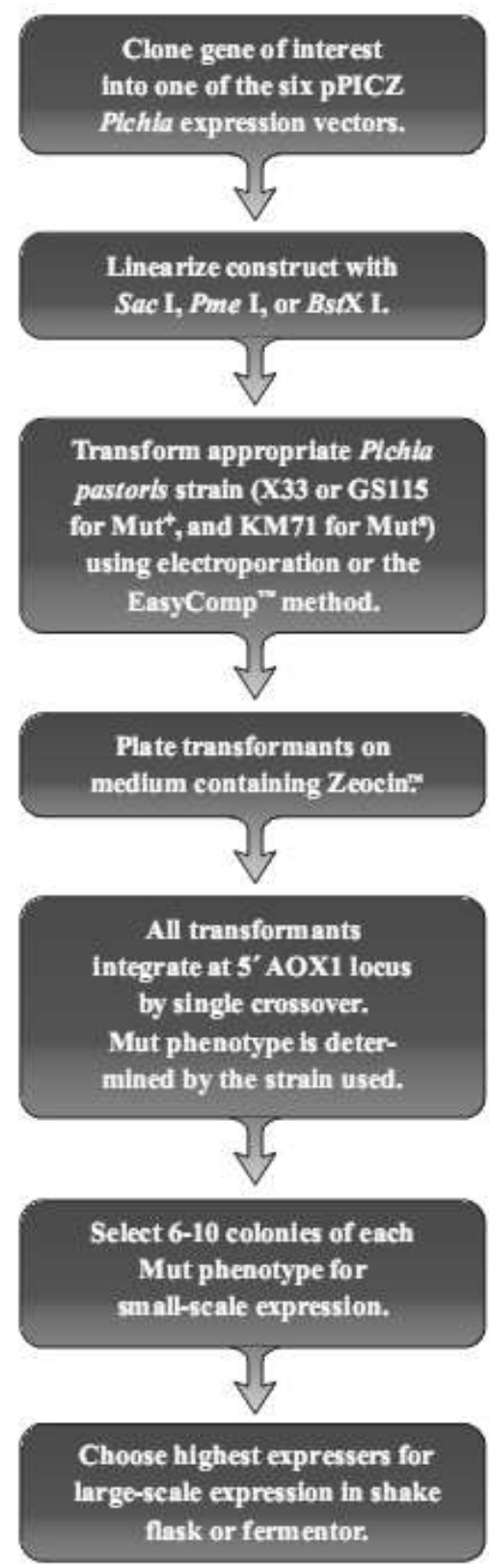

Figure 37. An overview process of Ecm 33 and Pst1 expressed $P$. pastoris generation

(Invitrogen, 2009) 


\subsection{Deglycosylation of $C g E \mathrm{~cm} 33$ and $C g P s t 1$ protein and western blot analysis}

In experiment 3.6.3 protein deglycosylation, we want to know to exact molecular weight of Ecm33 after deglycosylation. Therefore, we performed the same experiment and used the western blot. The result showed that anti-Ecm33 monoclonal antibody from rabbit can recognize the glycosylation (Figure 38).

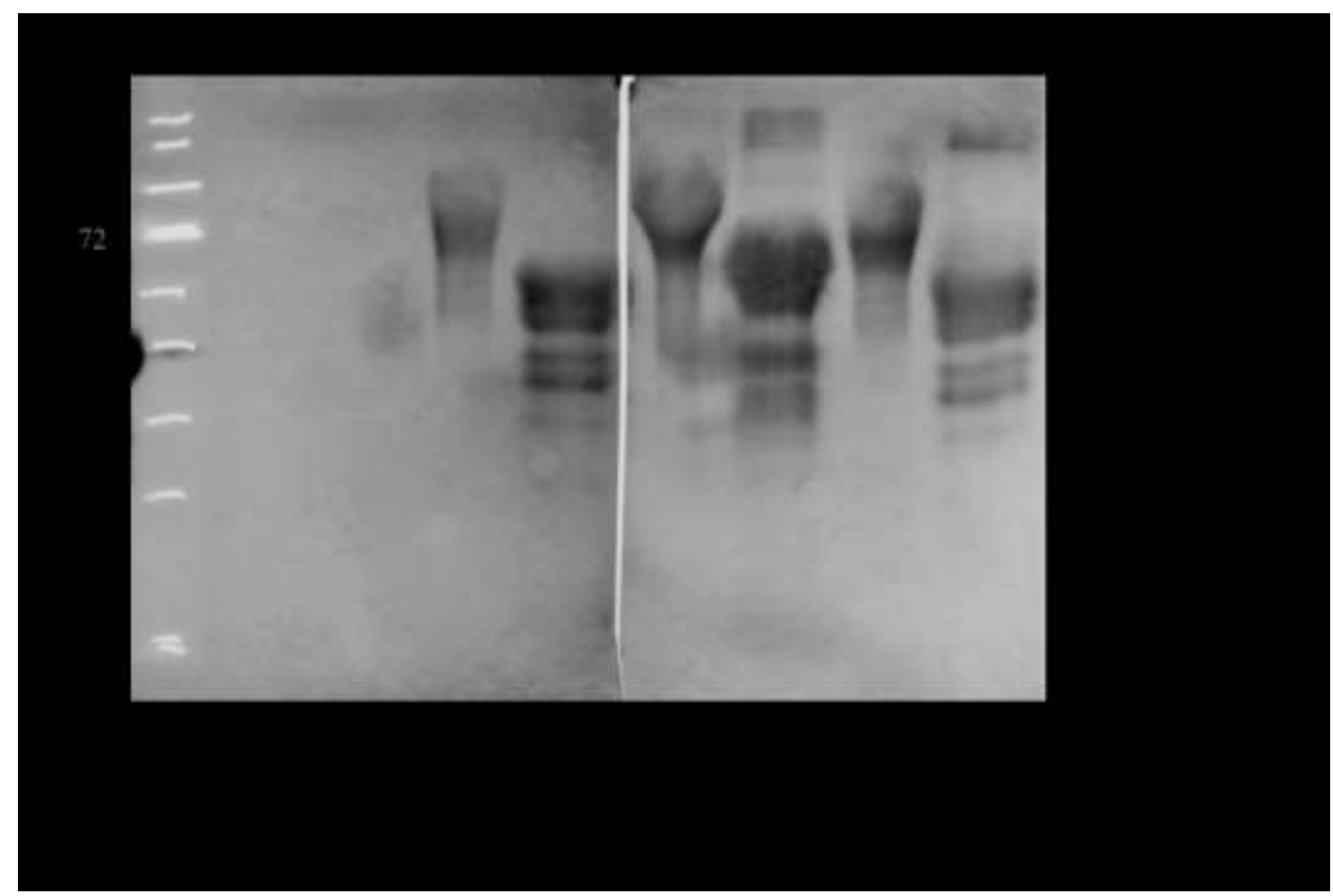

Figure 38. Deglycosylation of $C g E \mathrm{~cm} 33$ and $C g P s t 1$ proteins and Western blot analysis

Western blot analysis after for $C g E c m 33$ and $C g$ Pst1 proteins deglycosylation by double digestion of $\mathrm{N}$-glycosidae and O-glycosidase (N/O: $\mathrm{CgEcm} 33$ or $C g P s t 1$ was incubated with both glycosidase). Lane 1: protein marker, lane 2: CgPst1 without treatment, lane 3: $C g$ Pst1 with $\mathrm{N}$ - and O-glycosidase, lane 4: $C g E c m 33$ without treatment, lane 5: $C g E c m 33$ with N- and Oglycosidase, lane 6: $C g$ Pstl without treatment, lane 7: $C g$ Pst1 with N-and O-glycosidase, lane 8: $C g E c m 33$ without treatment, and lane 9: $C g E c m 33$ with $\mathrm{N}$ - and O-glycosidase. The serum rabbit no. 22 was tested in lane 2-5 and serum rabbit no. 23 was tested in lane 6-9 with titer 1:4,000. 


\subsection{The preliminary results of competitive inhibition assay}

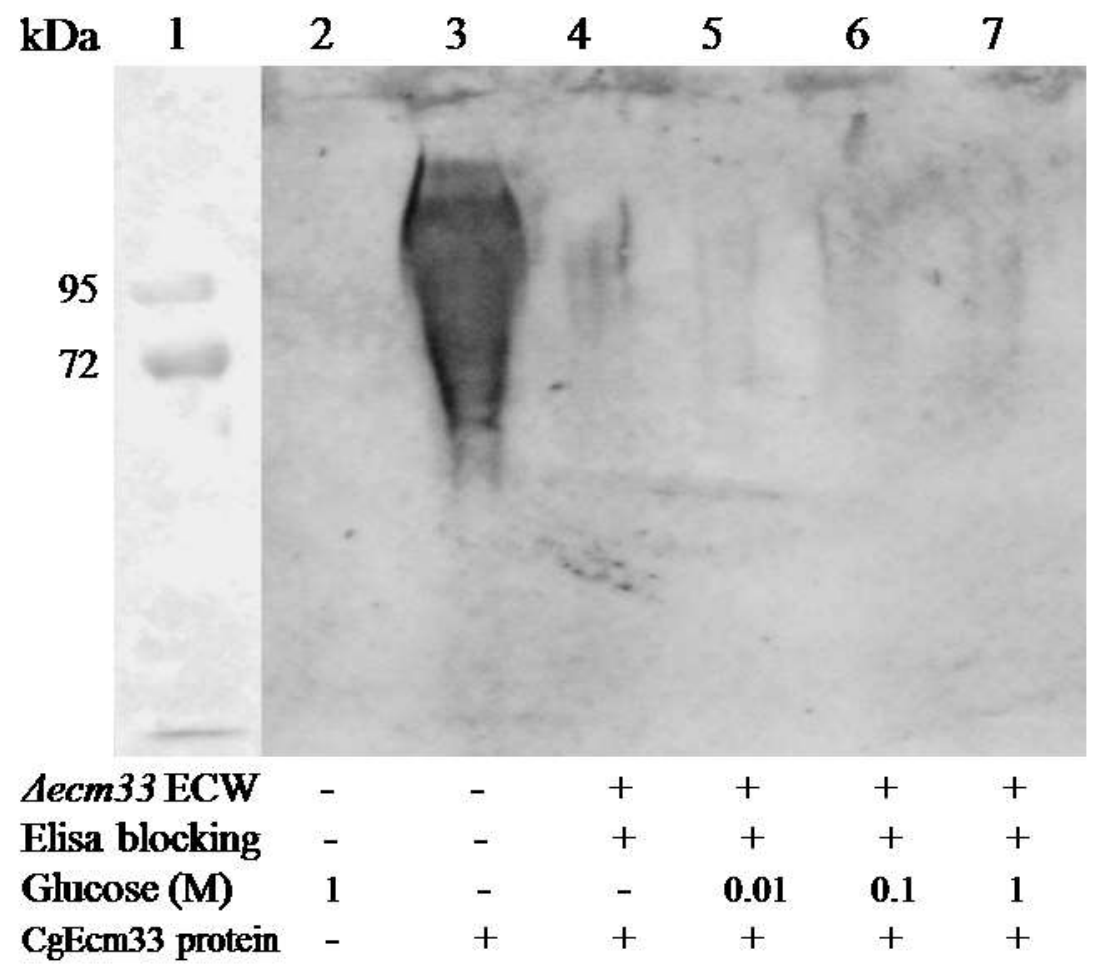

Figure 39. Glucose competitive inhibition assay

$20 \mu \mathrm{l}$ of the extracted cell walls (ECWs; $20 \mathrm{mg} / \mathrm{ml}$ ) were incubated with ELISA blocking solution, incubated with or without glucose $(10 \mu 1$ of $0.01,0.1$, or $1 \mathrm{M})$ and then added Ecm33 protein $(250 \mu \mathrm{g} / \mathrm{ml})$ and washed to remove unbound protein. SDS-PAGE electrophoresis and western blot analysis were performed to determine binding complex.

Lane 1: protein marker, lane 2: $1 \mathrm{M}$ of glucose, lane 3: Ecm33 protein from P. pastoris, lane 4: co-incubation without glucose competitor, lane 5: co-incubation with $0.01 \mathrm{M}$ glucose competitor, lane 6: co-incubation with $0.1 \mathrm{M}$ glucose competitor, and lane 7: co-incubation with $1 \mathrm{M}$ glucose competitor (lane 4-7 are co-incuabion between $C g$ Ecm33 expressed in P. pastoris with $\Delta e c m 33$ ECWs, and glucose was used to prevent binding between $C g$ Ecm 33 expressed in $P$. pastoris with $\Delta e c m 33$ ECWs) 


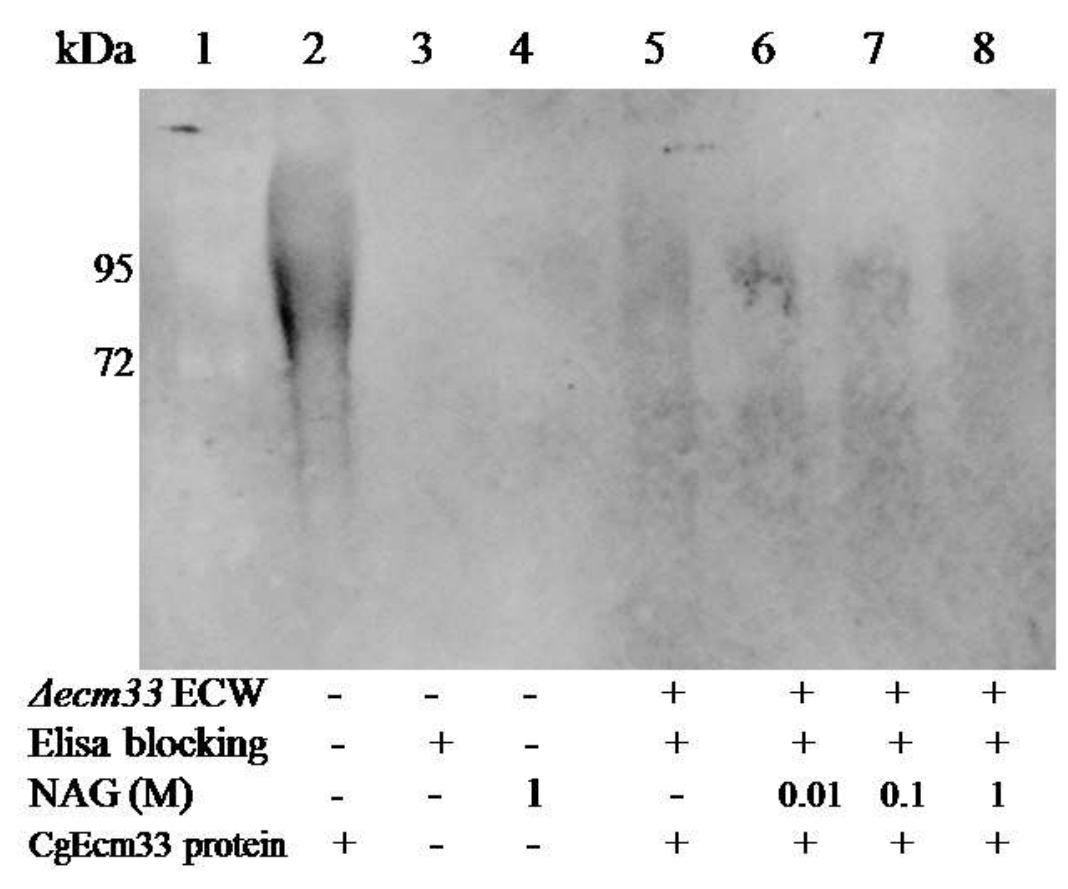

Figure 40. $\mathrm{N}$-acetylglucosamine competitive inhibition assay

$20 \mu \mathrm{l}$ of the extracted cell walls (ECWs; $20 \mathrm{mg} / \mathrm{ml}$ ) were incubated with ELISA blocking solution, incubated with or without $\mathrm{N}$-acetylglucosamine (NAG; $10 \mu 1$ of $0.01,0.1$, or $1 \mathrm{M}$ ) and then added Ecm33 protein $(250 \mu \mathrm{g} / \mathrm{ml})$ and washed to remove unbound protein. SDS-PAGE electrophoresis and western blot analysis were performed to determine binding complex.

Lane 1: protein marker, lane 2: $1 \mathrm{M}$ of $\mathrm{N}$-acetylglucosamine, lane 3: Ecm33 protein from $P$. pastoris, lane 4: co-incubation without $\mathrm{N}$-acetylglucosamine competitor, lane 5: co-incubation with $0.01 \mathrm{M} \mathrm{N}$-acetylglucosamine competitor, lane 6: co-incubation with $0.1 \mathrm{M} \mathrm{N}$ acetylglucosamine competitor, and lane 7: co-incubation with $1 \mathrm{M} \mathrm{N}$-acetylglucosamine competitor (lane 4-7 are co-incuabion between $C g$ Ecm33 expressed in P. pastoris with $\Delta e c m 33$ ECWs, and $\mathrm{N}$-acetylglucosamine was used to prevent binding between $C g \mathrm{Ecm} 33$ expressed in $P$. pastoris with $\triangle e c m 33 \mathrm{ECWs)}$ 


\section{REFERENCES}

Bader, O., Schwarz, A., et al. (2012). "Gross Karyotypic and Phenotypic Alterations among Different Progenies of the Candida glabrata CBS138/ATCC2001 Reference Strain." PLoS One 7(12): e52218.

Bom, I. J., Dielbandhoesing, S. K., et al. (1998). "A new tool for studying the molecular architecture of the fungal cell wall: one-step purification of recombinant trichoderma beta-(1-6)-glucanase expressed in Pichia pastoris." Biochim Biophys Acta 1425(2): 419424.

Borg-von Zepelin, M., Kunz, L., et al. (2007). "Epidemiology and antifungal susceptibilities of Candida spp. to six antifungal agents: results from a surveillance study on fungaemia in Germany from July 2004 to August 2005." J Antimicrob Chemother 60(2): 424-428.

Brunke, S. and Hube, B. (2012). "Two unlike cousins: Candida albicans and C. glabrata infection strategies." Cell Microbiol.

Busscher, H. J., Rinastiti, M., et al. (2010). "Biofilm formation on dental restorative and implant materials." J Dent Res 89(7): 657-665.

Chabane, S., Sarfati, J., et al. (2006). "Glycosylphosphatidylinositol-anchored Ecm33p influences conidial cell wall biosynthesis in Aspergillus fumigatus." Appl Environ Microbiol 72(5): 3259-3267.

Colombo, A. L., Guimaraes, T., et al. (2007). "Prospective observational study of candidemia in Sao Paulo, Brazil: incidence rate, epidemiology, and predictors of mortality." Infect Control Hosp Epidemiol 28(5): 570-576.

Csank, C. and Haynes, K. (2000). "Candida glabrata displays pseudohyphal growth." FEMS Microbiol Lett 189(1): 115-120.

Das, I., Nightingale, P., et al. (2011). "Epidemiology, clinical characteristics, and outcome of candidemia: experience in a tertiary referral center in the UK." Int J Infect Dis 15(11): e759-763.

de Groot, P. W., Bader, O., et al. (2013). "Adhesins in human fungal pathogens: glue with plenty of stick." Eukaryot Cell. 
de Groot, P. W., de Boer, A. D., et al. (2004). "Proteomic analysis of Candida albicans cell walls reveals covalently bound carbohydrate-active enzymes and adhesins." Eukaryot Cell 3(4): 955-965.

de Groot, P. W., Kraneveld, E. A., et al. (2008). "The cell wall of the human pathogen Candida glabrata: differential incorporation of novel adhesin-like wall proteins." Eukaryot Cell 7(11): 1951-1964.

de Groot, P. W., Ruiz, C., et al. (2001). "A genomic approach for the identification and classification of genes involved in cell wall formation and its regulation in Saccharomyces cerevisiae." Comp Funct Genomics 2(3): 124-142.

Desai, C., Mavrianos, J., et al. (2011). "Candida glabrata Pwp7p and Aed1p are required for adherence to human endothelial cells." FEMS Yeast Res 11(7): 595-601.

Diezmann, S., Cox, C. J., et al. (2004). "Phylogeny and evolution of medical species of Candida and related taxa: a multigenic analysis." J Clin Microbiol 42(12): 5624-5635.

Domergue, R., Castano, I., et al. (2005). "Nicotinic acid limitation regulates silencing of Candida adhesins during UTI." Science 308(5723): 866-870.

Dujon, B., Sherman, D., et al. (2004). "Genome evolution in yeasts." Nature 430(6995): 35-44.

EUCAST (2008). "EUCAST definitive document EDef 7.1: method for the determination of broth dilution MICs of antifungal agents for fermentative yeasts." Clin Microbiol Infect 14(4): 398-405.

Fermentas (2009). First Strand cDNA Synthesis Kit \#K1611, \#K1612.

Ferrari, S., Sanguinetti, M., et al. (2011). "Contribution of CgPDR1-regulated genes in enhanced virulence of azole-resistant Candida glabrata." PLoS One 6(3): e17589.

Fidel, P. L., Jr., Vazquez, J. A., et al. (1999). "Candida glabrata: review of epidemiology, pathogenesis, and clinical disease with comparison to C. albicans." Clin Microbiol Rev 12(1): 80-96.

Fukuda, Y., Tsai, H. F., et al. (2013). "Transcriptional Profiling of Candida glabrata during Phagocytosis by Neutrophils and in the Infected Mouse Spleen." Infect Immun 81(4): 1325-1333.

Gietz, R. D. and Schiestl, R. H. (2007). "High-efficiency yeast transformation using the LiAc/SS carrier DNA/PEG method." Nat Protoc 2(1): 31-34. 
Giri, S. and Kindo, A. J. (2012). "A review of Candida species causing blood stream infection." Indian J Med Microbiol 30(3): 270-278.

Gow, N. A., van de Veerdonk, F. L., et al. (2012). "Candida albicans morphogenesis and host defence: discriminating invasion from colonization." Nat Rev Microbiol 10(2): 112-122.

Hobson, R. P., Munro, C. A., et al. (2004). "Loss of cell wall mannosylphosphate in Candida albicans does not influence macrophage recognition." J Biol Chem 279(38): 3962839635.

Invitrogen (2009). EasySelect ${ }^{\mathrm{TM}}$ Pichia Expression Kit: For Expression of Recombinant Proteins Using pPICZ and pPICZ $\alpha$ in Pichia pastoris.

Jin, C. (2012). "Protein Glycosylation in Aspergillus fumigatus Is Essential for Cell Wall Synthesis and Serves as a Promising Model of Multicellular Eukaryotic Development." Int J Microbiol 2012: 654251.

Kapteyn, J. C., Van Egmond, P., et al. (1999). "The contribution of the O-glycosylated protein Pir2p/Hsp150 to the construction of the yeast cell wall in wild-type cells and beta 1,6glucan-deficient mutants." Mol Microbiol 31(6): 1835-1844.

Kaur, R., Domergue, R., et al. (2005). "A yeast by any other name: Candida glabrata and its interaction with the host." Curr Opin Microbiol 8(4): 378-384.

Klis, F. M., Brul, S., et al. (2010). "Covalently linked wall proteins in ascomycetous fungi." Yeast 27(8): 489-493.

Kraneveld, E. A., de Soet, J. J., et al. (2011). "Identification and differential gene expression of adhesin-like wall proteins in Candida glabrata biofilms." Mycopathologia 172(6): 415427.

Lesage, G., Tremblay, M., et al. (2001). "Mechanism of Kex2p inhibition by its proregion." FEBS Lett 508(3): 332-336.

Leventakos, K., Lewis, R. E., et al. (2010). "Fungal infections in leukemia patients: how do we prevent and treat them?" Clin Infect Dis 50(3): 405-415.

Liao, Y., Chen, M., et al. (2013). "Epidemiology of opportunistic invasive fungal infections in China: review of literature." Chin Med J (Engl) 126(2): 361-368.

Lin-Cereghino, J., Wong, W. W., et al. (2005). "Condensed protocol for competent cell preparation and transformation of the methylotrophic yeast Pichia pastoris." Biotechniques 38(1): 44, 46, 48. 
Ma, B., Pan, S. J., et al. (2007). "Assimilation of $\mathrm{NAD}(+)$ precursors in Candida glabrata." $\underline{\text { Mol }}$ Microbiol 66(1): 14-25.

Marchetti, O., Bille, J., et al. (2004). "Epidemiology of candidemia in Swiss tertiary care hospitals: secular trends, 1991-2000." Clin Infect Dis 38(3): 311-320.

Martinez-Lopez, R., Monteoliva, L., et al. (2004). "The GPI-anchored protein CaEcm33p is required for cell wall integrity, morphogenesis and virulence in Candida albicans." Microbiology 150(Pt 10): 3341-3354.

Martinez-Lopez, R., Nombela, C., et al. (2008). "Immunoproteomic analysis of the protective response obtained from vaccination with Candida albicans ecm33 cell wall mutant in mice." Proteomics 8(13): 2651-2664.

Martinez-Lopez, R., Park, H., et al. (2006). "Candida albicans Ecm33p is important for normal cell wall architecture and interactions with host cells." Eukaryot Cell 5(1): 140-147.

Mayer, F. L., Wilson, D., et al. (2013). "Candida albicans pathogenicity mechanisms." Virulence 4(2): 119-128.

Noble, S. M. and Johnson, A. D. (2005). "Strains and strategies for large-scale gene deletion studies of the diploid human fungal pathogen Candida albicans." Eukaryot Cell 4(2): 298309.

Odani, T., Shimma, Y., et al. (1996). "Cloning and analysis of the MNN4 gene required for phosphorylation of N-linked oligosaccharides in Saccharomyces cerevisiae." Glycobiology 6(8): 805-810.

Orta-Zavalza, E., Guerrero-Serrano, G., et al. (2013). "Local silencing controls the oxidative stress response and the multidrug resistance in Candida glabrata." Mol Microbiol.

Pardo, M., Monteoliva, L., et al. (2004). "PST1 and ECM33 encode two yeast cell surface GPI proteins important for cell wall integrity." Microbiology 150(Pt 12): 4157-4170.

Perlroth, J., Choi, B., et al. (2007). "Nosocomial fungal infections: epidemiology, diagnosis, and treatment." Med Mycol 45(4): 321-346.

Pfaller, M. A., Diekema, D. J., et al. (2010). "Results from the ARTEMIS DISK Global Antifungal Surveillance Study, 1997 to 2007: a 10.5-year analysis of susceptibilities of Candida Species to fluconazole and voriconazole as determined by CLSI standardized disk diffusion." J Clin Microbiol 48(4): 1366-1377.

Qiagen (2003). The QIAexpressionist. 
Qiagen (2006). RNeasy Mini Handbook.

Richter, S. S., Galask, R. P., et al. (2005). "Antifungal susceptibilities of Candida species causing vulvovaginitis and epidemiology of recurrent cases." J Clin Microbiol 43(5): 2155-2162.

Roche (2007). LightCycler ${ }^{\mathbb{B}}$ FastStart DNA Master ${ }^{\text {PLUS }}$ SYBR Green I.

Roetzer, A., Gabaldon, T., et al. (2011). "From Saccharomyces cerevisiae to Candida glabratain a few easy steps: important adaptations for an opportunistic pathogen." FEMS Microbiol Lett 314(1): 1-9.

Romano, J., Nimrod, G., et al. (2006). "Disruption of the Aspergillus fumigatus ECM33 homologue results in rapid conidial germination, antifungal resistance and hypervirulence." Microbiology 152(Pt 7): 1919-1928.

Rouabhia, M., Semlali, A., et al. (2012). "Disruption of the ECM33 gene in Candida albicans prevents biofilm formation, engineered human oral mucosa tissue damage and gingival cell necrosis/apoptosis." Mediators Inflamm 2012: 398207.

Sambrook, J., Russell, D.W. (2001). Molecular Cloning: A Laboratory Manual. New York, Cold Spring Harbor Laboratory Press.

Schmidt, P. (2007). Molecular mechanisms of the human pathogen Candida glabrata involved in the interaction with the host. Molecular Biology Program, Faculty of Biology. Goettingen, Georg August University Goettingen. Ph.D.

Schwarzmüller, T. (2009). Drug resistance and virulence of the human fungal pathogen Candida glabrata. Genetic-Microbiology. Vienna, University of Vienna. Ph.D.

Shen, J., Guo, W., et al. (2005). "CaNAT1, a heterologous dominant selectable marker for transformation of Candida albicans and other pathogenic Candida species." Infect Immun 73(2): 1239-1242.

Sifuentes-Osornio, J., Corzo-Leon, D. E., et al. (2012). "Epidemiology of Invasive Fungal Infections in Latin America." Curr Fungal Infect Rep 6(1): 23-34.

Wach, A. (1996). "PCR-synthesis of marker cassettes with long flanking homology regions for gene disruptions in S. cerevisiae." Yeast 12(3): 259-265.

Walker, C. A., Gomez, B. L., et al. (2010). "Melanin externalization in Candida albicans depends on cell wall chitin structures." Eukaryot Cell 9(9): 1329-1342.

Weig, M., Haynes, K., et al. (2001). "A GAS-like gene family in the pathogenic fungus Candida glabrata." Microbiology 147(Pt 8): 2007-2019. 
Weig, M., Jansch, L., et al. (2004). "Systematic identification in silico of covalently bound cell wall proteins and analysis of protein-polysaccharide linkages of the human pathogen Candida glabrata." Microbiology 150(Pt 10): 3129-3144.

Wu, S. and Letchworth, G. J. (2004). "High efficiency transformation by electroporation of Pichia pastoris pretreated with lithium acetate and dithiothreitol." Biotechniques 36(1): 152-154.

Zupancic, M. L., Frieman, M., et al. (2008). "Glycan microarray analysis of Candida glabrata adhesin ligand specificity." Mol Microbiol 68(3): 547-559. 


\section{PUBLICATION}

During the Ph.D. study, two papers have been published in PLOS ONE and Medical Mycology Journal.

- Bader, O., Schwarz, A., Kraneveld, E., Tangwattanachuleeporn, M., Schmidt, P., Jacobsen, M., Gross, U., De Groot, P., and Weig, M. (2012). Gross karyotypic and phenotypic alterations among different progenies of the Candida glabrata CBS138/ATCC2001 reference strain. PLoS ONE 7 (12), e52218.

- Tangwattanachuleeporn, M., Somparn, P., Poolpol, K., Gross, U., Weig, M., and Bader, O. (2013). Prevalence and Antifungal susceptibility of Cryptococcus neoformans Isolated from Pigeon Excreta in Chon Buri Province, Eastern Thailand. Med. Mycol. J. Vol. 54.

Other is in process to publish.

- Tangwattanachuleeporn, M., Bader, O., Gross, U., De Groot, P., and Weig, M. (2013). Characterization of the cell wall protein Ecm33 family in Candida glabrata. (in preparation). 


\section{CURRICULUM VITAE}

NAME

NATIONALITY

DATE OF BIRTH

PLACE OF BIRTH

PRESENT ADDRESS

HOME ADDRESS

E-mail

\section{EDUCATION}

1999-2003

2003-2005

2009-2013
Mr. Marut Tangwattanachuleeporn

Thai

18 March 1981

Bangkok, Thailand

Am Vogelsang 3, 37075 Göttingen, Germany

816/14 Moo.12, Bangna Road, Bangna 10260, Thailand

tp.marut@gmail.com

B.Sc. Microbiology

(Burapha University, Chon Buri, Thailand)

M.Sc. Medical Microbiology, the highest GPA in the class with

3.94 (Chulalongkorn University, Bangkok, Thailand)

Dr.rer.nat Biology

(Institute for Medical Microbiology, Georg-August University, Göttingen, Germany)

\section{EMPLOYMENT AND AFFILIATIONS}

Feb 2006-Mar 2009

Apr 2009-present

Oct 2011-present

Apr 2012-May 2013
Lecturer in the Department of Medical Science, Faculty of

Science, Burapha University, Chon Buri, Thailand

Lecturer in Faculty of Allied Health Sciences, Burapha University, Chon Buri, Thailand

The Young International Society for Human and Animal Mycology (ISHAM) representative for Southeast Asia Vice-President for Academic Affairs, Thai Student Association in Germany under the Patronage of HM the King (TSVD) 